TRANSACTIONS OF THE

AMERICAN MATHEMATICAL SOCIETY

Volume 349, Number 11, November 1997, Pages 4265-4310

S 0002-9947(97)01638-3

\title{
SEMIDIRECT PRODUCTS OF REGULAR SEMIGROUPS
}

\author{
PETER R. JONES AND PETER G. TROTTER
}

\begin{abstract}
Within the usual semidirect product $S * T$ of regular semigroups $S$ and $T$ lies the set $\operatorname{Reg}(S * T)$ of its regular elements. Whenever $S$ or $T$ is completely simple, $\operatorname{Reg}(S * T)$ is a (regular) subsemigroup. It is this 'product' that is the theme of the paper. It is best studied within the framework of existence (or e-) varieties of regular semigroups. Given two such classes, $\mathbf{U}$ and $\mathbf{V}$, the e-variety $\mathbf{U} * \mathbf{V}$ generated by $\{\operatorname{Reg}(S * T): S \in \mathbf{U}, T \in \mathbf{V}\}$ is well defined if and only if either $\mathbf{U}$ or $\mathbf{V}$ is contained within the e-variety $\mathbf{C S}$ of completely simple semigroups. General properties of this product, together with decompositions of many important e-varieties, are obtained. For instance, as special cases of general results the e-variety $L \mathbf{I}$ of locally inverse semigroups is decomposed as $\mathbf{I} * \mathbf{R Z}$, where $\mathbf{I}$ is the variety of inverse semigroups and $\mathbf{R Z}$ is that of right zero semigroups; and the e-variety $\mathbf{E S}$ of $E$-solid semigroups is decomposed as $\mathbf{C R} * \mathbf{G}$, where $\mathbf{C R}$ is the variety of completely regular semigroups and $\mathbf{G}$ is the variety of groups.

In the second half of the paper, a general construction is given for the e-free semigroups (the analogues of free semigroups in this context) in a wide class of semidirect products $\mathbf{U} * \mathbf{V}$ of the above type, as a semidirect product of e-free semigroups from $\mathbf{U}$ and $\mathbf{V}$, "cut down to regular generators". Included as special cases are the e-free semigroups in almost all the known important e-varieties, together with a host of new instances. For example, the e-free locally inverse semigroups, $E$-solid semigroups, orthodox semigroups and inverse semigroups are included, as are the e-free semigroups in such sub-e-varieties as strict regular semigroups, $E$-solid semigroups for which the subgroups of its self-conjugate core lie in some given group variety, and certain important varieties of completely regular semigroups. Graphical techniques play an important role, both in obtaining decompositions and in refining the descriptions of the e-free semigroups in some e-varieties.

Similar techniques are also applied to describe the e-free semigroups in a different 'semidirect' product of e-varieties, recently introduced by Auinger and Polák. The two products are then compared.
\end{abstract}

The semidirect product has a venerable history in semigroup theory. In conjunction with the wreath product, it has played a central role in the decomposition theory of finite semigroups. In the context of regular semigroups, variations on the usual product have been introduced for inverse semigroups and, recently, for locally inverse semigroups. We take a different approach, by studying the set $\operatorname{Reg}(S * T)$ of regular elements of the usual semidirect product of regular semigroups $S$ and $T$. In many important cases, these elements form a (regular) subsemigroup of $S * T$. These cases are most easily interpreted in the framework of e-varieties.

Received by the editors August 15, 1994.

1991 Mathematics Subject Classification. Primary 20M17, 20M07.

The authors are indebted to the Australian Research Council for their support of this research. The first author also gratefully acknowledges the support of National Science Foundation grant INT-8913404. 
An e-variety (sometimes called existence variety or bivariety) is a class of regular semigroups that is closed under products, quotients and regular subsemigroups. For an introduction to this topic see $\S 1$. We show (Theorem 3.1) that for e-varieties $\mathbf{U}, \mathbf{V}$, the property that $\operatorname{Reg}(S * T)$ is a subsemigroup of $S * T$ for every $S \in \mathbf{U}, T \in$ $\mathbf{V}$ (under a standard assumption on the actions) holds if and only if either $\mathbf{U}$ or $\mathbf{V}$ is contained within the e-variety $\mathbf{C S}$ of completely simple semigroups. While at first sight this may seem unduly restrictive, it turns out that all the 'well-known' e-varieties fall within its compass and, moreover, this result sets the stage for the use of the semidirect product to define operators on the lattice of e-varieties. We define the semidirect product of e-varieties $\mathbf{U} * \mathbf{V}$ as the e-variety generated by all semigroups of the form $\operatorname{Reg}(S * T), S \in \mathbf{U}, T \in \mathbf{V}$, whenever $\mathbf{U}$ or $\mathbf{V}$ is contained in CS. Alternatively, it is generated by the semigroups of regular elements of wreath products, in a similar way. Many interesting properties of this product are determined in $\S 3$.

The second main purpose of this study is to use the semidirect product to investigate the e-free semigroups in various e-varieties. The $e$-free semigroup $E F \mathbf{V}_{X}$ on the set $X$, in the e-variety $\mathbf{V}$, plays a role analogous to that of the free semigroup on $X$ in a variety (see $\S 1$ ). However, e-free semigroups of rank more than one exist only in e-varieties contained within either $L \mathbf{I}$ or $\mathbf{E S}$, the e-varieties of locally inverse and $E$-solid semigroups, respectively. In $\S 3$ it is determined exactly when $\mathbf{U} * \mathbf{V}$ lies within either $L \mathbf{I}$ or ES.

To this end we first identify the result of the semidirect product operation on some important e-varieties. Then in $\S 4$ we prove that $L \mathbf{I}$ has the simple decomposition $\mathbf{I} * \mathbf{R Z}$, where $\mathbf{I}$ is the (e-) variety of inverse semigroups and $\mathbf{R Z}$ is the (e-) variety of right zero semigroups. Some well known sub-e-varieties of $L \mathbf{I}$ have similar decompositions. In $\S 5$, we prove that ES also has a simple decomposition, as $\mathbf{C R} * \mathbf{G}$ (first proved by M. Szendrei [35]), where $\mathbf{C R}$ is the (e-) variety of completely regular semigroups and $\mathbf{G}$ is the (e-) variety of groups. This decomposition is a very special case of the equality of the semidirect product $\mathbf{U} * \mathbf{K}$ with the Mal'cev product $\mathbf{U m K}$ for certain ('e-local') $E$-solid e-varieties $\mathbf{U}$ and for any group variety $\mathbf{K}$; and of the description of $\mathbf{U} * \mathbf{G}$, for $\mathbf{U}$ completely regular, as the class $C_{\infty} \mathbf{U}$ of all $E$-solid semigroups whose self-conjugate cores belong to $\mathbf{U}$ (ES being $C_{\infty} \mathbf{C R}$ ). On the other hand, the semidirect product $\mathbf{G} * \mathbf{V}$, again for $\mathbf{V}$ completely regular (but not contained in $\mathbf{G}$ ), is just the variety $\mathbf{L} \mathbf{G m} \mathbf{V}$, also consisting of completely regular semigroups. The operator $\mathbf{V} \rightarrow \mathbf{V}^{T_{l}}=\mathbf{L G m} \mathbf{V}$ and its dual $\mathbf{V} \rightarrow \mathbf{V}^{T_{r}}=\mathbf{R G m V}$, (which can be described using 'reverse' semidirect products), have been intensively studied. (Here $\mathbf{L G}$ and $\mathbf{R G}$ are the e-varieties of left and of right groups, respectively).

The heart of the paper is a general technique for describing the e-free semigroup on $X$ in the semidirect product $\mathbf{U} * \mathbf{V}$ of e-varieties $\mathbf{U}, \mathbf{V}$. It was noted above that such semigroups exist, for rank more than one, only if the product lies within $L \mathbf{I}$ or ES. In the general scheme, $E F(\mathbf{U} * \mathbf{V})_{X}$ is a regular subsemigroup of the semidirect product of a certain e-free semigroup in $\mathbf{U}$ with $E F \mathbf{V}_{X}$. The reader familiar with products of group varieties [28] will recognize this general form, as will those familiar with products of semigroup varieties. (In fact we have presented in $\S 6$ a brief summary of the latter situation, as a guide for the sequel, since the proof method we use does not appear to be in the literature). However, considerable difficulties arise when regularity must be retained at all stages. 
In $\S 7.1$ we describe $E F \mathbf{E S}_{X}$ as the regular subsemigroup of the semidirect product $E F \mathbf{C R}_{F \mathbf{G}_{X} \times X} * F \mathbf{G}_{X}$ that is generated by $\{((1, y), y): y \in \bar{X}\}$, where $\bar{X}$ is a 'doubling' of the generators $X$. ('Cutting down to regular generators' refers to focusing on this subsemigroup of the semidirect product.) Since the e-free completely regular semigroups are well described [14], we obtain a relatively simple solution to the 'word problem' in $E F \mathbf{E S}_{X}$. In fact, this semigroup has recently been described by Szendrei [35]; however, our technique also determines the e-free semigroup on $X$ in any e-variety $C_{\infty} \mathbf{C R}(\mathbf{H})$, where $\mathbf{C R}(\mathbf{H})$ is the e-variety comprising all completely regular semigroups, the subgroups of which belong to the group variety $\mathbf{H}$; and also determines the e-free semigroups in certain e-varieties of orthodox semigroups.

In $\S 8$ we describe $E F(\mathbf{U} * \mathbf{V})_{X}$ for any variety $\mathbf{U}$ of inverse semigroups and any e-variety $\mathbf{V}$ such that this semigroup exists, by a similar construction. As a very special case we obtain in $\S 8.1$ a description of $E F L \mathbf{I}_{X}$ as a regular subsemigroup of the semidirect product of a free inverse semigroup and the e-free right zero semigroup on $X$ (essentially the set $\bar{X}$ with right zero multiplication). Our description is inherently different from those of Auinger [3, 4], which are based, implicitly, on his decomposition $L \mathbf{I}=\mathbf{S} * \mathbf{C S}$, where $\mathbf{S}$ is the variety of semilattices. Descriptions of the e-free semigroups in certain sub-e-varieties (such as strict regular semigroups) follow similarly.

In $\S 8.2$ we describe $E F \mathbf{V}^{T_{l}}$ for any completely regular variety $\mathbf{V}$, in a similar way, embedded in the semidirect product of a free group with an e-free semigroup from $\mathbf{V}$. A variation of the technique also yields the free semigroup in that variety. The word problem is solvable in this free semigroup, modulo that in $F \mathbf{V}_{X}$. The solution is much more transparent than that found by Kadourek [17].

In $\S 9$ we provide a unified form of this approach. We have chosen not to present the general theorem first, because its proof essentially breaks into the cases already considered and because the description presented is more complicated than in any of the individual cases.

Graphical methods, in particular semigroupoids ('categories without identities'), play a useful role in the paper. A graphical description of $E F \mathbf{E S}_{X}$ is given in $\S 7.3$ that is similar to that of Szendrei [35] (and ultimately derives from work of Margolis and Pin [22] on inverse semigroups). It has the decided advantage, over the purely semigroup-theoretic description, of providing a set of canonical forms for the elements of the e-free semigroup (rather than just solving the 'word problem'). Our description also extends to the other $E$-solid cases described above. We have been unable, however, to find such a graphical interpretation in the cases described in $\S 8$.

Graphical methods also prove useful in obtaining the decompositions in the first part of the paper. In particular, 'regular' versions of the Derived Category Theorem of [37] are used in both parts of $\S 5$.

The final section examines briefly an alternative semidirect product of e-varieties, studied by Polák [32] and Auinger and Polák [5], generalizing certain semidirect products of varieties of inverse semigroups. Their product relies on a variant multiplication; it is well defined as long as the second factor is locally inverse. We show how a technique similar to the above yields a quite simple description of the e-free semigroups in such products, where defined, and we compare their product with the one on which we have focused in this paper. 
For general reference on semigroups see [11]; on e-varieties the surveys [10, 13, 41]; and on graphical techniques in semigroup theory [37]. As a general rule, we use the terms 'free' and 'e-free' where others might use 'relatively free' or 'relatively e-free'; the meaning will be clear from the context. The term 'bifree' has often been used instead of e-free. Of necessity, the next two sections contain a large amount of preparatory material. The reader may prefer to begin in $\S 3$ and make reference to $\S \S 1$ and 2 when necessary.

\section{BACKGROUND ON REGULAR SEMIGROUPS AND E-VARIETIES}

For any element $x$ of a semigroup $S$, the set of inverses of $x$ is denoted by $V(x)$; $S$ is regular if $V(x)$ is nonempty for all $x$. If $x$ lies in a subgroup of $S$ then $x^{-1}$ and $x^{0}$ denote the inverse of $x$ and the identity element, respectively, in $H_{x}$ (the $\mathcal{H}$-class of $x$ ). The set of idempotents of $S$ is denoted $E_{S}$ and its set of regular elements $\operatorname{Reg} S$. In general Reg $S$ is not a subsemigroup of $S$. Hall [7] gave the following useful criterion.

Result 1.1. For an arbitrary semigroup $S, \operatorname{Reg} S \leq S$ if and only if the product of any pair of idempotents is regular.

The following related result, due to Nambooripad [27, Theorem 1.2], will be needed in the sequel.

Lemma 1.2. Let $S$ be a regular semigroup, let $s_{1}, \ldots, s_{n} \in S$ and let $s_{1}^{\prime} \in V\left(s_{1}\right)$, $s_{n}^{\prime} \in V\left(s_{n}\right)$. Then $V\left(s_{1} \cdots s_{n}\right) \cap s_{n}^{\prime} S s_{1}^{\prime} \neq \emptyset$.

Let $S$ and $T$ be arbitrary semigroups. A relational morphism $\phi: S \rightarrow T$ is a fully defined relation from $S$ to $T$ such that $s_{1} \phi s_{2} \phi \subseteq\left(s_{1} s_{2}\right) \phi$ for all $s_{1}, s_{2} \in S ; \phi$ is a division if for distinct $s_{1}, s_{2} \in S, s_{1} \phi$ and $s_{2} \phi$ are disjoint. The graph \# $\phi$ of $\phi$ is the associated subsemigroup of $S \times T$. Now suppose $S$ and $T$ are regular. The relational morphism $\phi$ is regular if its graph is a regular subsemigroup of $S \times T$ or, equivalently, if whenever $s \in S$ and $t \in s \phi$ then there exist $s^{\prime} \in V(s)$ and $t^{\prime} \in V(t)$ such that $t^{\prime} \in s^{\prime} \phi$.

We say a regular semigroup $S$ regularly divides a regular semigroup $T$ if there is a regular subsemigroup $R$ of $T$ and a morphism of $R$ onto $S$. It will be seen in the next section, as a consequence of results on regular semigroupoids, that $S$ regularly divides $T$ if and only if there is a regular division $S \rightarrow T$.

We turn now to the study of classes of regular semigroups. An e-variety of regular semigroups is a class of regular semigroups that is closed under homomorphic images, direct products and regular subsemigroups, or equivalently, in view of the preceding paragraph, closed under homomorphic images and regular division.

Let us list some of the e-varieties that will be encountered in the sequel. The class of all regular semigroups will be denoted $\mathbf{R}$, and that of trivial semigroups by T.

Inverse semigroups form an e-variety, denoted I. In fact, inverse semigroups form a variety of 'unary semigroups', that is, semigroups with an additional unary operation. As classes of regular semigroups, the e-varieties contained in $\mathbf{I}$ are precisely the varieties of inverse semigroups. We shall use the terms 'variety' and 'e-variety' interchangeably in this context. See [31] for a survey of inverse semigroup varieties. Of particular importance here will be SG, the class of Clifford semigroups, or semilattices of groups, and its sub-e-varieties $\mathbf{S H}$, comprising the semilattices of groups 
from the group varieties $\mathbf{H}$. The e-variety of semilattices is denoted $\mathbf{S}$, that of all groups $\mathbf{G}$.

In a similar way, completely regular semigroups form an e-variety, denoted $\mathbf{C R}$, and also a variety of unary semigroups, its subvarieties being again the same as its sub-e-varieties. The e-variety of completely simple semigroups is denoted $\mathbf{C S}$; and LZ, RZ, RB, LG, RG denote respectively the e-varieties of left zero semigroups, right zero semigroups, rectangular bands, left groups and right groups. For any group variety $\mathbf{H}, \mathbf{C R}(\mathbf{H})$ comprises the completely regular semigroups all of whose subgroups belong to $\mathbf{H}$ (and similarly for $\mathbf{C S}(\mathbf{H})$ ). In particular, $\mathbf{C R}(\mathbf{T})$ is the e-variety of bands, denoted $\mathbf{B}$.

The local monoids of a regular semigroup $S$ are the subsemigroups $e S e$, for $e \in E_{S}$. Each is again regular. Given a class $\mathbf{C}$ of regular semigroups, $S$ is said to be locally in $\mathbf{C}$ if each of its local monoids belongs to $\mathbf{C}$.

If $\mathbf{U}$ is any e-variety of regular semigroups, let $L \mathbf{U}$ be the class of regular semigroups that are locally in $\mathbf{U}$. Then [8] $L \mathbf{U}$ is again an e-variety and the operator $\mathbf{U} \rightarrow L \mathbf{U}$ is a closure operator on the lattice of e-varieties. Among the examples above, $\mathbf{C S}(\mathbf{H})$ is closed for every group variety $\mathbf{H}$, since it is just $L \mathbf{H}$. Further examples of importance include $L \mathbf{S G}$ and $L \mathbf{S}$, traditionally known as the classes of strict regular and combinatorial strict regular semigroups and denoted $\mathbf{S R}$ and CSR, respectively. Their intersections with I are denoted SI and CSI, respectively. Their common intersection with $\mathbf{B}$ is the variety $\mathbf{N B}$ of normal bands.

The e-variety $L \mathbf{I}$ of locally inverse semigroups has played a major role in the history of regular semigroups and, not surprisingly, does so in this paper.

The core $C(S)$ of a regular semigroup $S$ is the subsemigroup generated by its idempotents. It is well known that $C(S)$ is a regular subsemigroup of $S$. The selfconjugate core $C_{\infty}(S)$ of $S$ is the subsemigroup formed by taking the union of the following sequence of subsemigroups: let $C_{0}(S)=C(S)$ and, for $i>1$, let $C_{i}(S)$ be the subsemigroup generated by $\left\{x^{\prime} a x^{\prime}: a \in C_{i-1}, x \in S, x^{\prime} \in V(x)\right\}$. That is, $C_{\infty}(S)$ is the smallest subsemigroup that contains $E_{S}$ and contains $x a x^{\prime}$ whenever it contains $a$, for any mutually inverse elements $x, x^{\prime}$ of $S$. Again it is true that $C_{\infty}(S)$ is a regular subsemigroup of $S$.

For $\mathbf{U}$ an e-variety of regular semigroups, let $C \mathbf{U}$ be the class of regular semigroups whose cores belong to $\mathbf{U}$; and let $C_{\infty} \mathbf{U}$ be the class whose self-conjugate cores belong to $\mathbf{U}$. Again [8] the operator $\mathbf{U} \rightarrow C \mathbf{U}$ is a closure operator on the lattice of e-varieties. However, this is not the case for the operator $\mathbf{U} \rightarrow C_{\infty} \mathbf{U}$, as Zhang [43] has shown. Among the examples above, $\mathbf{G}$ and $\mathbf{C S}$ are closed with respect to each operator, since $\mathbf{G}=C \mathbf{T}=C_{\infty} \mathbf{T}$ and $\mathbf{C S}=C \mathbf{C S}=C_{\infty} \mathbf{C S}$, as is easily verified. In addition, $\mathbf{I}=C \mathbf{S}=C_{\infty} \mathbf{S}$. Further examples of importance are $C \mathbf{B}=C_{\infty} \mathbf{B}$, which is the e-variety of orthodox semigroups, denoted $\mathbf{O}$, and $C \mathbf{N B}=C_{\infty} \mathbf{N B}$, the e-variety of generalized inverse semigroups, denoted GI.

The e-variety $\mathbf{E S}=C \mathbf{C R}$ of $E$-solid semigroups has also played a major role in regular semigroup theory and continues to do so here. By [38, Corollary 5.2], $\mathbf{E S}=C_{\infty} \mathbf{C R}$. Lest the reader think that the operators $C$ and $C_{\infty}$ are identical, S. Zhang [43] showed that $C_{\infty} \mathbf{C S}(\mathbf{A}) \subset C \mathbf{C S}(\mathbf{A})$, where $\mathbf{A}$ is the variety of abelian groups.

The following observation will be useful in the sequel. The converse inclusion is false, as is seen by considering the case $\mathbf{U}=\mathbf{T}$.

Proposition 1.3. For any e-variety $\mathbf{U}$ of regular semigroups, $C L \mathbf{U} \subseteq L C \mathbf{U}$. 
Proof. Let $S \in C L \mathbf{U}$, so that $C(S) \in L \mathbf{U}$ and $e C(S) e \in \mathbf{U}$ for all $e \in E_{S}$. Now since $E_{e S e} \subseteq e E_{S} e \subseteq e C(S) e, C(e S e) \subseteq e C(S) e$, whence $C(e S e) \in \mathbf{U}$ and $e S e \in C \mathbf{U}$ for all $e \in E_{S}$. Hence $S \in L C \mathbf{U}$.

A concept of central importance in the study of e-varieties is the idea of an e-free semigroup on a set $X$ in an e-variety $\mathbf{V}$. Let $F$ be the free semigroup on the set $\bar{X}=X \cup X^{\prime}$, where $X^{\prime}=\left\{x^{\prime} \mid x \in X\right\}$ is a set in one-one correspondence with, and disjoint from, $X$. Put $x^{\prime \prime}=x$, for $x \in X$. A mapping $\phi$ of $\bar{X}$ into a regular semigroup $S$ is matched if $a \phi$ and $a^{\prime} \phi$ are mutually inverse elements of $S$ for every $a \in \bar{X}$. An e-free semigroup on $X$ in $\mathbf{V}$ is a member $T$ of $\mathbf{V}$ together with a matched mapping $\iota: \bar{X} \rightarrow T$, with the property that for any matched mapping $\phi: \bar{X} \rightarrow S \in \mathbf{V}$, there is a unique morphism $\bar{\phi}: T \rightarrow S$ such that $\iota \bar{\phi}=\phi$. It is easily seen that if such an object exists it is unique up to isomorphism. We then denote it $E F \mathbf{V}_{X}$.

This concept was introduced by J. Kadourek and M. Szendrei [19] for e-varieties of orthodox semigroups (using the terminology 'bifree'). Y.T. Yeh [42] extended the definition to cover e-varieties in general and determined those e-varieties of regular semigroups which possess e-free semigroups on all nonempty sets, as follows.

Result 1.4. An e-variety of regular semigroups possesses an e-free semigroup on every nonempty set if and only if it is contained in either LI or ES.

The e-variety $L \mathbf{E S}$ is a natural envelope for the e-varieties encountered in this paper, so we shall attempt to extend our results to that setting when feasible. Of course $L \mathbf{E S}$ does not possess e-free semigroups in general.

In $\S 7$, we shall describe in concrete terms the e-free semigroups in certain evarieties of $E$-solid semigroups, in terms of those in associated completely regular semigroup varieties and of relatively free groups. In [14] the first author described the e-free semigroups in $\mathbf{C R}(\mathbf{H})$, where $\mathbf{H}$ is a group variety. Although we shall not need the details of that description, we shall need the framework in which it lies.

A unary semigroup is a semigroup endowed with a unary operation. This operation will usually be denoted by inversion. Unary semigroups play a central role in the general theory of e-varieties ([8]), although here we consider them only in a limited context.

Let $X$ be a nonempty set. Let ( and $)^{-1}$ be two symbols disjoint from $\bar{X}$ and put $\breve{X}=\bar{X} \cup\left\{(,)^{-1}\right\}$. The free unary semigroup $X^{u}$ on $\bar{X}$ is the smallest subsemigroup of the free semigroup $\breve{X}^{+}$that contains $\bar{X}$ and contains $(u)^{-1}$ whenever it contains $u$. Its members are unary words over $\bar{X}$. It has been observed that completely regular semigroups may be regarded as unary semigroups. The e-free completely regular semigroup on $X$ was described in [14]. First, it is the quotient of $X^{u}$ by the semigroup congruence $\rho_{\mathbf{C R}}$ generated by the pairs

$$
\left(u(u)^{-1} u, u\right),\left(u(u)^{-1},(u)^{-1} u\right),\left(\left((u)^{-1}\right)^{-1}, u\right), u \in X^{u},
$$

together with the pairs

$$
\left(x x^{\prime} x, x\right),\left(x^{\prime} x x^{\prime}, x^{\prime}\right), x \in X .
$$

Extending the technique of J. Kadourek and L. Polák [18], an algorithm was given in [14, Theorem 4] to decide whether or not two unary words over $\bar{X}$ belong to the congruence $\rho_{\mathbf{C R}}$. More generally, given any completely regular semigroup variety $\mathbf{U}$, there is a congruence $\rho_{\mathbf{U}}$ such that $X^{u} / \rho_{\mathbf{U}} \cong E F \mathbf{U}_{X}$, namely the least congruence 
such that the quotient belongs to $\mathbf{U}$. For $\mathbf{U}=\mathbf{C R}(\mathbf{H})$, the following result [14, Theorem 6] holds.

Result 1.5. Let $\mathbf{H}$ be a variety of groups and let $X$ be a nonempty set. There is a procedure for deciding whether or not two unary words over $\bar{X}$ belong to $\rho_{\mathbf{C R}(\mathbf{H})}$. The procedure is effective if the word problem in the free group $F \mathbf{H}_{X}$ is soluble.

We may call this decision problem the word problem for $\operatorname{EFCR}(\mathbf{H})_{X}$ (or, similarly, for the e-free semigroup on $X$ in any completely regular semigroup variety). This problem is therefore decidable as long as the same is true in the free groups over $\mathbf{H}$.

We now consider some further properties of locally inverse and $E$-solid semigroups. Their key role is clear from Result 1.4. In [42], Yeh also showed that various analogues of properties normally possessed by varieties, in the usual sense, carry over to e-varieties in those situations (but not generally). We have, for instance, the following, the first part of which is [42, Corollary 4.7] and the second part of which is a combination of Theorem 2.1, Lemma 3.1.3 and Lemma 3.2.3 from the same paper.

Result 1.6. (i) Let $\mathbf{C}$ be a class of regular semigroups that is contained in either $L \mathbf{I}$ or $\mathbf{E S}$. Then the e-variety generated by $\mathbf{C}$ comprises all regular semigroups that regularly divide a product of members of $\mathbf{C}$.

(ii) If $S$ is any E-solid or locally inverse semigroup, then for any subset $A$ of $S$ that contains an inverse for each of its members, there is a least regular subsemigroup of $S$ that contains $A$, denoted here by $\langle A\rangle_{e}$ and called the regular subsemigroup generated by $A$. Moreover, any morphisms from $S$ to a regular semigroup $T$ that agree on $A$ also agree on $\langle A\rangle_{e}$.

Lemma 1.7. In the terminology of (ii) of Result 1.6, if $\phi: S \rightarrow T$ is any morphism to an E-solid or locally inverse semigroup $T$, then $\langle A\rangle_{e} \phi=\langle A \phi\rangle_{e}$.

Proof. Let $B$ be any regular subsemigroup of $T$ that contains $A \phi$. By the proof of [42, Lemma 4.1], $\operatorname{Reg}\left(B \phi^{-1}\right)$ is a regular subsemigroup of $S$. (Yeh's hypothesis of surjectivity is not needed for this part of his proof). Clearly $\operatorname{Reg}\left(B \phi^{-1}\right)$ contains $A$ and, therefore, $\langle A\rangle_{e}$, whence $B$ contains $\langle A\rangle_{e} \phi$. Since $\langle A\rangle_{e} \phi$ is regular, the proof is complete.

Finally, the concept of the Mal'cev product of e-varieties has proven very fruitful in the contexts of varieties of inverse and, especially, completely regular semigroups. A recent result of the second author may be regarded as representing e-varieties of the form $C_{\infty} \mathbf{U}$ as certain such products. (For completely regular semigroup varieties, the corollary below was explicitly stated by Zhang [44].) First some terminology is needed.

Let $\mathbf{U}, \mathbf{V}$ be e-varieties. Their Mal'cev product $\mathbf{U m} \mathbf{V}$ is the e-variety generated by those regular semigroups $S$ that possess a congruence $\rho$ such that (a) $S / \rho \in \mathbf{V}$ and (b) each idempotent $\rho$-class belongs to $\mathbf{U}$.

Let $S$ be any regular semigroup. We say $S$ is $C_{\infty}$-unitary, if for all $s \in S$ and $a \in C_{\infty}(S)$, as $\in C_{\infty}(S)$ or $s a \in C_{\infty}(S)$ implies $s \in C_{\infty}(S)$. Then [39, Lemma 1.2] $C_{\infty}(S)$ is always contained in $E_{S} \sigma$, and equality holds if and only if $S$ is $C_{\infty^{-}}$ unitary. Here $\sigma$ is the least group congruence on $S$. A morphism $\phi: S \rightarrow T$ of regular semigroups is $C_{\infty}$-separating if it separates the elements of $C_{\infty}(S)$. If $T$ is a regular semigroup then a $C_{\infty}$-unitary cover of $T$ consists of a $C_{\infty}$-unitary 
regular semigroup $S$ together with a $C_{\infty}$-separating morphism $\phi$ of $S$ onto $T$; by [39, Lemma 1.1], $C_{\infty}(S) \phi=C_{\infty}(T)$ and, therefore, $C_{\infty}(S) \cong C_{\infty}(T)$.

Result 1.8 ([39]). Any regular semigroup has a $C_{\infty}$-unitary cover.

Corollary 1.9. For any e-variety $\mathbf{U}$ of regular semigroups, $C_{\infty} \mathbf{U}=\mathbf{U m G}$.

Proof. Suppose $S \in C_{\infty}$ U. Let $T$ be a $C_{\infty}$-unitary cover for $S$. As noted above, $C_{\infty}(T) \cong C_{\infty}(S)$. We may assume, without loss of generality, then, that $S$ itself is $C_{\infty}$-unitary. Let $\sigma$ be the least group congruence on $S$. As remarked above, $E_{S} \sigma=C_{\infty}(S) \in \mathbf{U}$. Hence $S \in \mathbf{U m G}$.

Conversely, if $S$ possesses a congruence $\rho$ such that $S / \rho \in \mathbf{G}$ and $E_{S} \rho$ (the unique idempotent $\rho$-class) belongs to $\mathbf{U}$, then since $C_{\infty}(S) \subseteq E_{S} \rho, S \in C_{\infty} \mathbf{U}$.

\section{Preliminaries ON SEMIGROUPOIDS}

Semigroupoids will play two related but distinct roles in the sequel: in the representation of $E$-solid e-varieties as semidirect products ( $\$ 5)$, and in the description of the e-free semigroups in such e-varieties $(\S 7)$. For an overview of their use in semigroup theory in general see [37, Appendix B]. Most of the following is from [14].

A graph $G$ is a pair $(V, E)$ of sets, together with a pair of maps $h, t: E \rightarrow V$. For $e \in E, h(e)$ is the head of $e$ and $t(e)$ is its tail. We write $V=V G$, the set of vertices of $G$, and $E=E G$, the set of edges of $G$. For each pair $(x, y)$ of vertices of $G, G(x, y)$ denotes the homset or edge set $\{u \in E: t(u)=x, h(u)=y\}$. We often write $u: x \rightarrow y$ instead of $u \in G(x, y)$. The edges in a given homset are said to be coterminal. A graph is strongly connected (or "bonded" in the terminology of [37]) if for any pair of distinct vertices $e$ and $f$ there is a path from $e$ to $f$.

A semigroupoid is a graph $C=(V, E)$ together with a map $m:\{(e, f) \in E \times E$ : $h(e)=t(f)\} \rightarrow E$ which we shall write as juxtaposition, such that the usual partial associativity law is satisfied. For any $c \in V, C(c, c)$ is a semigroup, the local semigroup at $c$, which we may denote by $C_{c}$. Note that $C_{c}$ may be empty.

A category is a semigroupoid $C$ with the additional property that for each vertex $c$ of $C$, there is an edge $1_{c} \in C_{c}$ such that $e 1_{c}=e$ for all $e: a \rightarrow c$ and $1_{c} f=f$ for all $f: c \rightarrow d, a, d \in V C$. The local semigroups then become monoids. The general relationship between categories and semigroupoids is like that between monoids and semigroups, and we leave to the reader the task of formulating analogues of the definitions and elementary results that follow. See [37] for an exposition of the role of categories in monoid theory.

A morphism $\phi: G \rightarrow H$ of graphs is a pair $\left(\phi_{V}, \phi_{E}\right)$ of functions $\phi_{V}: V G \rightarrow V H$ and $\phi_{E}: E G \rightarrow E H$ satisfying $h(e) \phi_{V}=h\left(e \phi_{E}\right)$ and $t(e) \phi_{V}=t\left(e \phi_{E}\right)$ for each edge $e$ of $G$. A morphism of semigroupoids is a morphism of the underlying graphs that respects composition. A morphism is faithful if it is injective on homsets, and quotient if it is bijective on $V$ and surjective on $E$.

A relation $\phi: G \rightarrow H$ of graphs is a pair $\left(\phi_{V}, \phi_{E}\right)$, consisting of a function $\phi_{V}: V G \rightarrow V H$ and a fully defined relation $\phi_{E}: E G \rightarrow E H$ such that $G(x, y) \phi_{E} \subseteq$ $H\left(x \phi_{V}, y \phi_{V}\right)$, for all vertices $x, y$ of $G$. Its graph \# $\phi$ is the subgraph of $G \times H$ with vertex and edge sets the graphs of $\phi_{V}$ and $\phi_{E}$, respectively. A relational morphism $\phi: C \rightarrow D$ of semigroupoids is a relation $\left(\phi_{V}, \phi_{E}\right)$ of the underlying graphs such that $e \phi_{E} f \phi_{E} \subseteq(e f) \phi_{E}$ for all consecutive edges $e, f$ of $C$ or, equivalently, such that $\# \phi$ is a subsemigroupoid of $C \times D$. A relational morphism is a division if for distinct 
coterminal edges $e, f$ of $C, e \phi_{E}$ and $f \phi_{E}$ are disjoint. As for semigroups, every faithful morphism is a division, the inverse relation of every quotient morphism is a division, and the composition of divisions is a division. A semigroupoid $C$ is said to divide a semigroupoid $D$ if there is a semigroupoid $E$, a quotient morphism $E \rightarrow C$ and a faithful morphism $E \rightarrow D$. Similarly to [37, Proposition 2.5], this occurs if and only if there is a division $C \rightarrow D$.

A congruence on a semigroupoid $C=(V, E)$ is an equivalence $\rho$ on $E$ that consists of coterminal pairs and is compatible with all well-defined products. The quotient semigroupoid $C / \rho=(V, E / \rho)$ is then a semigroupoid in the obvious way and the natural morphism $C \rightarrow C / \rho$ is a quotient morphism. Conversely, for every quotient morphism $C \rightarrow D$, the edge function induces a congruence on $C$. The usual isomorphism theorems then hold.

Given a family $\left(G_{i}\right)_{i \in I}$ of graphs, their product is the graph whose vertex and edge sets are, respectively, the Cartesian products of $\left(V G_{i}\right)_{i \in I}$ and $\left(E G_{i}\right)_{i \in I}$, with $h$ and $t$ defined componentwise. The product of semigroupoids is that of the underlying graphs, with componentwise operation.

The free semigroupoid $G^{+}$on a graph $G$ has as its vertex set $V G$ and as its edge set the nonempty paths in $G$, under concatenation. If $\theta: G \rightarrow C$ is a graph morphism to a semigroupoid $C$, then there is a unique semigroupoid morphism $\theta^{+}: G^{+} \rightarrow C$ extending $\theta$. There is an injection $\eta: E G^{+} \rightarrow(E G)^{+}$, where the latter is the free semigroup on $E G$, obtained by mapping each path $p$ in $G$ to the corresponding word in the alphabet $E G$. We may identify one-vertex graphs with their edge sets and one-vertex semigroupoids with their local semigroups, so $\eta$ may be treated as a faithful semigroupoid morphism $G^{+} \rightarrow(E G)^{+}$.

The weak derived semigroupoid $W_{\phi}$ of a surjective morphism $\phi: S \rightarrow T$ of semigroups consists of the graph with vertex set $T^{1}$ and edge set $T^{1} \times S$, setting $t\left(t_{1}, s\right)=t_{1}$ and $h\left(t_{1}, s\right)=t_{1}(s \phi)$, for $t_{1} \in T^{1}, s \in S$, together with the product $\left(t_{1}, s_{1}\right)\left(t_{2}, s_{2}\right)=\left(t_{1}, s_{1} s_{2}\right)$, defined whenever $t_{1}\left(s_{1} \phi\right)=t_{2}$. The derived semigroupoid $D_{\phi}$ of $\phi$ is the quotient $W_{\phi} / \equiv$, where $\left(t, s_{1}\right) \equiv\left(t, s_{2}\right)$ if $\left(t\left(s_{1} \phi\right)=t\left(s_{2} \phi\right)\right.$ and) $s s_{1}=s s_{2}$ for all $s \in t \phi^{-1}$. (If $T$ has no identity, then $1 \phi^{-1}$ is taken to consist of an adjoined identity for $S$ ). Denote the $\equiv$-class of $(t, s)$ by $[t, s]$. Our definitions and notation follow loosely those of Margolis [21], who, however, adheres to the categorical setting.

We turn now to regular semigroupoids. An edge $x$ of a semigroupoid $C$ is regular if there is an edge $a$ such that $x a x=x ; C$ itself is regular if each of its edges is: then, as for semigroups, for each edge $x$ the set $V(x)=\{a \in E C: x a x=x, a x a=a\}$ of inverses of $x$ is nonempty. As pointed out in [33, $\S 2]$, many of the usual properties of regular semigroups carry over to regular semigroupoids. For instance, if $\phi: C \rightarrow D$ is a quotient morphism of regular semigroupoids and $d, e$ are mutually inverse edges of $D$, then there exist mutually inverse edges $a, b$ of $C$ such that $a \phi=d$ and $b \phi=e$.

If $\phi: S \rightarrow T$ is a morphism of regular semigroups, then $W_{\phi}$ need not be a regular semigroupoid, in general. However, when $T$ is a group then $W_{\phi}$ is indeed regular, for if $(t, s) \in E W_{\phi}$, then $\left(t s \phi, s^{\prime}\right) \in V((t, s))$ for any $s^{\prime} \in V(s)$.

A relational morphism $\phi: C \rightarrow D$ of regular semigroupoids is regular if its graph is a regular subsemigroupoid of $C \times D$, that is, for all pairs $(e, f) \in \# \phi_{E}$, there exist inverses $e^{\prime}$ and $f^{\prime}$ of $e$ and $f$ in $E C$ and $E D$, respectively, such that $\left(e^{\prime}, f^{\prime}\right) \in \# \phi_{E}$.

We say that a regular semigroupoid $C$ regularly divides a regular semigroupoid $D$ if there is a regular semigroupoid $E$, a quotient morphism $E \rightarrow C$ and a faithful morphism $E \rightarrow D$. 
Result 2.1 ([14]). A regular semigroupoid $C$ regularly divides a regular semigroupoid $D$ if and only if there is a regular division $\phi: C \rightarrow D$.

For any semigroupoid $C$, let $\operatorname{Reg} C$ denote the subgraph with $V \operatorname{Reg} C=V C$ and $E \operatorname{Reg} C$ the set of regular edges of $C$. We write $\operatorname{Reg} C \leq C$ if $\operatorname{Reg} C$ is a subsemigroupoid of $C$.

An e-variety of regular semigroupoids is a class $\mathbf{V}$ of regular semigroupoids such that:

(i) $\mathbf{V}$ is closed under direct products;

(ii) if $C \in \mathbf{V}, D$ is a regular semigroupoid and there is a faithful morphism $D \rightarrow C$, then $D \in \mathbf{V}$;

(iii) if $C \in \mathbf{V}, D$ is a regular semigroupoid and there is a quotient morphism $C \rightarrow D$, then $D \in \mathbf{V}$.

Szendrei [33] introduced this concept (calling it a 'bivariety') for the case of orthodox semigroupoids: those regular semigroupoids all of whose local semigroups are orthodox. It is easy to see that these form such an e-variety. Other examples of e-varieties of semigroupoids include that of trivial semigroupoids - those with at most one edge per homset - and that of all regular semigroupoids.

With any e-variety $\mathbf{W}$ of regular semigroups we may associate two e-varieties of semigroupoids. Let $\mathbf{l W}$ consist of the regular semigroupoids all of whose local subsemigroups belong to $\mathbf{W}$. (For instance, $\mathbf{l O}$ consists of the orthodox semigroupoids.) It is easily verified that $\mathbf{I W}$ is an e-variety of semigroupoids (those that are locally $\mathbf{W}$ ) that contains $\mathbf{W}$. Let $\mathbf{g} \mathbf{W}$ be the e-variety of regular semigroupoids generated by $\mathbf{W}$. Thus $\mathbf{g W} \subseteq \mathbf{l} \mathbf{W}$.

Note that e-varieties of semigroupoids are closed under regular division. In contrast with the situation for semigroup varieties, it does not follow from the definitions that if $\mathbf{W}$ is an e-variety of regular semigroups then $\mathbf{g} \mathbf{W}$ consists of all regular semigroupoids that regularly divide a semigroup in W. For e-varieties contained in $\mathbf{E S}$ or $L \mathbf{I}$, however, $\mathbf{g W}$ is so definable, as the next result shows.

Result 2.2 ([14]). For any e-variety $\mathbf{W}$ of regular semigroups that is contained in either $\mathbf{E S}$ or LI, $\mathbf{g W}$ comprises all regular semigroupoids that regularly divide a semigroup in $\mathbf{W}$.

Call an e-variety $\mathbf{W}$ of regular semigroups e-local if $\mathbf{l W}=\mathbf{g W}$. In view of this last result, a sub-e-variety of $\mathbf{E S}$ or $L \mathbf{I}$ is e-local if and only if every regular semigroupoid that is locally $\mathbf{W}$ regularly divides a semigroup of $\mathbf{W}$.

Proposition 2.3. For any e-variety $\mathbf{W}$ of semigroups that contains $\mathbf{S}, C \mathbf{W}$ is e-local. In particular $\mathbf{I}, \mathbf{O}, \mathbf{E S}$ and $\mathbf{R}$ are all e-local.

Proof. Suppose $D$ is a locally $C \mathbf{W}$ regular semigroupoid. If $D$ is a semigroup, there is no problem. Otherwise, let $D_{c d}$ be its consolidation ([37]), which is the semigroup $E D \cup\{0\}$, where the product of elements of $E D$ is that in $D$, when defined, 0 otherwise, and the new element 0 acts as a zero. Clearly $D_{c d}$ is regular and the injection of $E D$ in $E D \cup\{0\}$ induces a regular division $D \rightarrow D_{c d}$.

Hence it suffices to prove $D_{c d} \in C \mathbf{W}$. Now since idempotents in the consolidation, other than 0 , must be loops of $D$, the core $C\left(D_{c d}\right)$ is just the subsemigroup $\bigcup\left\{C\left(D_{d}\right): d \in V D\right\} \cup\{0\}$. Let $Y$ be the semilattice $V D \cup\{0\}$, with $V D$ an antichain and 0 the zero element. Then $C\left(D_{c d}\right)$ is a strong semilattice composition of the semigroups $C\left(D_{d}\right), d \in V D$, and the trivial semigroup $\{0\}$, via $Y$. By [11, Proposition 5.12], since each $C\left(D_{d}\right) \in \mathbf{W}$ and $\mathbf{S} \subseteq \mathbf{W}, C\left(D_{c d}\right) \in \mathbf{W}$, as required. 
The final statement follows from the equations $\mathbf{I}=C \mathbf{S}, \mathbf{O}=C \mathbf{B}, \mathbf{E S}=C \mathbf{C R}$ and $\mathbf{R}=C \mathbf{R}$.

Szendrei [33] showed that the e-variety of orthodox semigroups is e-local. More importantly, she showed that every e-variety of orthodox completely regular semigroups is e-local. In particular, every e-variety of bands is e-local and every variety of groups is e-local.

Result 2.4 ([14]). For each variety $\mathbf{H}$ of groups the variety $\mathbf{C R}(\mathbf{H})$, comprising all completely regular semigroups whose subgroups belong to $\mathbf{H}$, is e-local.

We next consider e-free semigroupoids in e-varieties. Let $G$ be a graph. Denote by $G^{\prime}$ the graph with $V G^{\prime}=V G$ and $E G^{\prime}=(E G)^{\prime}$, where $(E G)^{\prime}$ is a set in one-one correspondence with $E G$ under $x^{\prime} \leftrightarrow x$; for $x^{\prime} \in(E G)^{\prime}, h\left(x^{\prime}\right)=t(x)$ and $t\left(x^{\prime}\right)=$ $h(x)$. Thus there are induced bijections between $G(a, b)$ and $G^{\prime}(b, a), a, b \in V G$. Let $\bar{G}=G \cup G^{\prime}$, that is, $\bar{G}$ is the graph with $V \bar{G}=V G$ and $E \bar{G}=E G \cup(E G)^{\prime}$. Following [33], a matched mapping from $\bar{G}$ to a regular semigroupoid $C$ is a graph morphism $\theta: \bar{G} \rightarrow C$ such that $x^{\prime} \theta \in V(x \theta)$ for all $x \in E \bar{G}$.

Let $\mathbf{V}$ be an e-variety of semigroupoids and $G$ a graph. An e-free semigroupoid on $G$ over $\mathbf{V}$ is a pair $(C, i)$, usually denoted simply $C$, where $C \in \mathbf{V}$ and $i$ : $\bar{G} \rightarrow C$ is a matched mapping, with the property that for every matched mapping $\theta: \bar{G} \rightarrow D \in \mathbf{V}$ there is a unique semigroupoid morphism $\bar{\theta}: C \rightarrow D$ such that $i \bar{\theta}=\theta$. By standard arguments, if such a semigroupoid exists then it is unique up to semigroupoid isomorphism. We then denote it $E F \mathbf{V}_{G}$.

The e-locality of $\mathbf{C R}(\mathbf{H})$ (Result 2.4) was proven in [14] by consideration of efree locally completely regular semigroupoids. These are examples of locally unary semigroupoids. We recall some details that are needed in the sequel.

A locally unary semigroupoid is a semigroupoid $C=(V, E)$ together with, for each $c \in V$, a unary operation $e \rightarrow e^{-1}$ on $C_{c}$. A unary morphism of locally unary semigroupoids is a semigroupoid morphism $\phi: C \rightarrow D$ such that for every loop $e$ of $C, e^{-1} \phi=(e \phi)^{-1}$. A congruence $\rho$ on a unary semigroupoid $C$ is unary if for any coterminal loops $u$ and $v, u \rho v$ implies $u^{-1} \rho v^{-1}$. The quotient semigroupoid is then a locally unary semigroupoid, and the usual isomorphism theorems again hold.

Let $G$ be a graph. For each vertex $x$ of $\bar{G}$ we first adjoin two loops at $x$, denoted $(\text { and })^{-1}$. (The same symbols will denote all instances of these loops, the meaning to be clear from context.) Let $\breve{G}$ denote the graph so obtained. Then $\breve{G}^{+}$is the free semigroupoid on $\breve{G}$. Define $G^{u}$ as follows. Its vertices are again those of $G$. Its edge set is the subset of $E \breve{G}^{+}$defined recursively by the following rules:

1. $x \in E G^{u}$ for every $x \in E \bar{G}$;

2. if $p, q \in E G^{u}$ and $h(p)=t(q)$ then $p q \in E G^{u}$; and if $p \in E G^{u}$ and $h(p)=t(p)$ then $(p)^{-1} \in E G^{u}$.

Thus $G^{u}$ is the smallest subsemigroupoid of $\breve{G}^{+}$that contains $\bar{G}$ and contains $(u)^{-1}$ whenever it contains a loop $u$. We call the edges of $G^{u}$ unary paths over $\bar{G}$. Clearly the symbols ( and $)^{-1}$ within a unary path $p$ come in matched pairs, the segment of $p$ lying within such a matched pair being a loop in $G^{u}$. It is straightforward to show that every graph morphism from $\bar{G}$ to a locally unary semigroupoid $C$ extends uniquely to a unary semigroupoid morphism from $G^{u}$ to $C$. Thus $G^{u}$ is the free locally unary semigroupoid on $\bar{G}$. Note that if $G$ is a onevertex graph, then $\breve{G}^{+}$may be identified with $\breve{E G^{+}}$, and $G^{u}$ reduces to the free unary semigroup on $\overline{E G}$. 
The morphism $\eta: \breve{G}^{+} \rightarrow \breve{E G}{ }^{+}$, introduced earlier, clearly maps unary paths over $\bar{G}$ to unary words over $E \bar{G}=\overline{E G}$. The restriction, which we again denote $\eta$, is therefore a faithful morphism $G^{u} \rightarrow(E G)^{u}$. Further, it is a clearly a unary morphism.

It should be pointed out that the free locally unary semigroupoid on $\bar{G}$ is not the same as the subsemigroupoid $U^{l o c}(G)$ of the 'free unary semigroupoid' on $G$ introduced by Szendrei [35, §3] in her treatment of e-free $E$-solid semigroups. This is most easily seen in the case of one-vertex graphs. In that situation, $U^{l o c}(G)$ reduces to the free unary semigroup on $E G$ (where she denotes the unary operation by $u \rightarrow u^{\prime}$ ), whilst $G^{u}$ reduces to the free unary semigroup on $\overline{E G}$. This distinction will be of some concern in $\S 7.3$.

\section{SEMidirect PRODUCTS OF E-VARIETIES}

Let $S$ and $T$ be semigroups. A (left) action of $T$ on $S$ is a map $S \times T \rightarrow S$, $(s, t) \rightarrow{ }^{t} s$ satisfying: (i) ${ }^{t_{1} t_{2}} s={ }^{t_{1}}\left({ }^{t_{2}} s\right)$ and (ii) ${ }^{t}\left(s_{1} s_{2}\right)={ }^{t} s_{1}{ }^{t} s_{2}$, for all $t, t_{1}, t_{2} \in$ $T$ and $s, s_{1}, s_{2} \in S$. If $T$ is a monoid, the action is left unitary if ${ }^{1} s=s$ for all $s \in S$. The semidirect product $S * T$ of $S$ and $T$, with respect to this action, has as its underlying set $S \times T$, with multiplication defined by $\left(s_{1}, t_{1}\right)\left(s_{2}, t_{2}\right)=\left(s_{1}{ }^{t_{1}} s_{2}, t_{1} t_{2}\right)$. It is well known that $S * T$ is a semigroup. It is trivially verified that the idempotents in $S * T$ are the pairs $(s, t)$ such that $t \in E_{T}$ and $s^{t} s=s$.

Following S. Eilenberg [6] and B. Tilson [37], the wreath product $S \mathrm{wr} T$ of semigroups $S$ and $T$ is defined to be the semidirect product $S^{T^{1}} * T$, where for $t \in T$ and $\alpha: T^{1} \rightarrow S,{ }^{t} \alpha$ is defined by $t_{1}{ }^{t} \alpha=\left(t_{1} t\right) \alpha$, for $t_{1} \in T$. (Some authors instead take the semidirect product of $S^{T}$ and $T$. The definition used here has the advantage that any semidirect product of $S$ and $T$ can be embedded in $S \mathrm{wr} T$, as long as the action is left unitary whenever $T$ is a monoid (cf. [37, pp. 190-191]).)

In this section we study 'products' of e-varieties of regular semigroups $\mathbf{U}$ and $\mathbf{V}$ associated with semidirect products $S * T, S \in \mathbf{U}, T \in \mathbf{V}$. In such constructions we shall always assume that if $T$ is a monoid then its action on $S$ is left unitary (although this is not an a priori assumption when studying products of individual semigroups; we shall see below that in many situations the assumption is not strictly needed). Now the semidirect product of regular semigroups need not again be regular, as simple examples will verify. (Indeed, in the context of individual semigroups Nico [26] and Skornyakov [36] have studied the question of regularity.) In Theorem 3.2 we determine for exactly which e-varieties $\mathbf{U}, \mathbf{V}$ it is true that every semidirect product $S * T, S \in \mathbf{U}, T \in \mathbf{V}$, is regular (under the assumption above): in this situation the e-variety generated by all such semidirect products is an interesting and useful object, as has been demonstrated previously (e.g. for completely regular varieties $[16])$.

We study, however, a more general construction. It may be the case that for every $S \in \mathbf{U}$ and every $T \in \mathbf{V}$, the set $\operatorname{Reg}(S * T)$ of regular elements of $S * T$ is a (regular) subsemigroup. In this situation we define the semidirect product $\mathbf{U} * \mathbf{V}$ of e-varieties $\mathbf{U}$ and $\mathbf{V}$ as the e-variety generated by $\{\operatorname{Reg}(S * T): S \in \mathbf{U}, T \in \mathbf{V}\}$.

Clearly, since every e-variety contains a trivial monoid $\{e\}$, say, and since for any regular semigroup $S,\{e\} \times S \cong S$ and $S \times\{e\} \cong S, \mathbf{U} * \mathbf{V}$ contains both $\mathbf{U}$ and $\mathbf{V}$, whenever the product is well defined.

In the definition of $\mathbf{U} * \mathbf{V}$, as mentioned above we require that if $T$ is a monoid then the action is left unitary. This restriction guarantees that if $S \in \mathbf{U}$ and $T$ 
is a trivial semigroup, then the only semidirect product $\operatorname{Reg}(S * T)$ that belongs to $\mathbf{U} * \mathbf{V}$ is $\operatorname{Reg}(S \times T) \cong S$. Hence $\mathbf{U} * \mathbf{T}=\mathbf{U}$. It turns out, however, that if $\mathbf{V} \nsubseteq \mathbf{G}$ then the restriction is unnecessary, since the e-variety generated by all such semidirect products yields no new semigroups. For in that case $\mathbf{V}$ contains some non-monoid $V$, say. If $T$ is any monoid of $\mathbf{V}$, then for any idempotent $f$ of $V$, the map $t \rightarrow(t, f)$ embeds $T$ in the non-monoid $T \times V \in \mathbf{V}$. Now if $T$ acts in any manner at all on $S \in \mathbf{U}$, then $\operatorname{Reg}(S * T)$ embeds in $\operatorname{Reg}(S *(T \times V))$, via the map $(s, t) \rightarrow(s,(t, f))$, where $T \times V$ acts on $S$ by ${ }^{(t, v)} s={ }^{t} s$. (That the restriction is essential otherwise is seen by considering examples such as $\mathbf{G} * \mathbf{T}=\mathbf{G}$. If a trivial semigroup $T=\{e\}$ acts on a group $G$ by ${ }^{e} g=1$ for all $g \in G$ (so non-left unitarily), then $G * T$ is a left zero semigroup of cardinality $|G|$.)

The following theorem determines exactly when $\mathbf{U} * \mathbf{V}$ is defined.

Theorem 3.1. Let $\mathbf{U}$ and $\mathbf{V}$ be e-varieties of regular semigroups. The property that $\operatorname{Reg}(S * T)$ is a (regular) subsemigroup of $S * T$ for every $S \in \mathbf{U}, T \in \mathbf{V}$ (with left unitary action whenever $T$ is a monoid), holds if and only if either $\mathbf{U} \subseteq \mathbf{C S}$ or $\mathbf{V} \subseteq \mathbf{C S}$.

Proof. (i) It is well known that any e-variety that is not contained within CS contains the two element semilattice and, therefore, all semilattices. To prove necessity, then, it suffices to show that there is a semidirect product of two semilattices, the regular elements of which do not form a subsemigroup. Let $S=\{e, f, 0\}$ be the semilattice with $e, f$ incomparable, and let $T=\{0,1\}$ be the two element chain, with $0<1$. Let $\alpha, \beta: T \rightarrow S$ be defined by $0 \alpha=e, 1 \alpha=f, 0 \beta=1 \beta=f$. Then in $S \mathrm{wr} T,(\alpha, 1)$ and $(\beta, 0)$ are idempotents. Now $(\alpha, 1)(\beta, 0)=(\alpha \beta, 0)$, where $0 \alpha \beta=0,1 \alpha \beta=f$, but for any $(\gamma, g) \in S$ wr $T, 1\left(\alpha \beta^{0} \gamma^{0}(\alpha \beta)\right)=0$, so that $(\alpha \beta, 0)(\gamma, g)(\alpha \beta, 0) \neq(\alpha \beta, 0)$; that is, $(\alpha, 1)(\beta, 0) \notin \operatorname{Reg}(S * T)$.

(ii) Let $S$ be any regular semigroup and let $T \in \mathbf{C S}$ act on $S$. (We do not assume the action is left unitary, even when $T$ is a monoid, during the proof.) By Result 1.1, it suffices to show that $E_{S * T} E_{S * T} \subseteq \operatorname{Reg}(S * T)$. We show, in fact, that $E_{S * T}(S * T) \subseteq \operatorname{Reg}(S * T)$.

So let $(x, e) \in E_{S * T}$ and $(y, h) \in S * T$. Thus $e \in E_{T}$ and $x^{e} x=x$. Let $(x y)^{\prime} \in V(x y)$. The reader may verify that $\left({ }^{(e h)^{-1} e}(x y)^{\prime},(e h)^{-1} e\right)$ is an inverse for $(x, e)(y, h)$. (The complete simplicity of $T$ comes into play via the equation $\left.e h(e h)^{-1} e=e.\right)$

(iii) Now let $S \in \mathbf{C S}$ and let $T$ be an arbitrary regular semigroup acting on $S$. (Again we make no assumptions on the action.) This time we show that $(S * T) E_{S * T} \subseteq \operatorname{Reg}(S * T)$.

So let $(x, t) \in S * T$ and $(y, f) \in E_{S * T}$. Thus $f \in E_{T}$ and $y=y^{f} y$. Let $(t f)^{\prime} \in V(t f)$ and $\left(x^{t} y\right)^{\prime} \in V\left(x^{t} y\right)$. Then $\left({ }^{(t f)^{\prime}}\left(x^{t} y\right)^{\prime},(t f)^{\prime}\right)$ is an inverse for $(x, t)(y, f)$. We outline the calculation involved. We have

$$
\left(x^{t} y, t f\right)\left({ }^{(t f)^{\prime}}\left(x^{t} y\right)^{\prime},(t f)^{\prime}\right)\left(x^{t} y, t f\right)=\left(x^{t} y^{t f(t f)^{\prime}}\left(\left(x^{t} y\right)^{\prime} x^{t} y\right), t f\right) .
$$

Now the idempotent $\left(x^{t} y\right)^{\prime} x^{t} y$ is $\mathcal{L}$-related to ${ }^{t} y$, and so since the action is by endomorphisms of $S,{ }^{t f(t f)^{\prime}}\left(\left(x^{t} y\right)^{\prime} x^{t} y\right)$ is an idempotent $\mathcal{L}$-related to ${ }^{t f(t f)^{\prime} t} y=$ ${ }_{t f(t f)^{\prime} t}\left(y^{f} y\right.$ ), which in turn is $\mathcal{L}$-related to ${ }^{t f} y$ and thus to $x^{t} y$ (since $x^{t} y=$ $\left.x^{t} y^{t f} y\right)$. Hence the product above yields $\left(x^{t} y, t f\right)$. The other requisite equation is straightforwardly verified. 
Two observations are in order. First, there is a certain curious duality to the two proofs of sufficiency, in each case slightly stronger than is necessary for the result at hand: if $T$ is completely simple then $E_{S * T}(S * T) \subseteq \operatorname{Reg}(S * T)$, and if $S$ is completely simple then $(S * T) E_{S * T} \subseteq \operatorname{Reg}(S * T)$.

Second, the statement of the theorem remains true without the restrictions on the actions. This is no longer the case when considering the question of when $S * T$ itself is regular, to which allusion was made earlier. For completeness, we prove the following analogue of the preceding theorem. That the semidirect product is always regular when one factor or the other is a group (and the alternate factor is regular) is folklore.

Theorem 3.2. Let $\mathbf{U}$ and $\mathbf{V}$ be e-varieties of regular semigroups. The property that $S * T$ is regular for every $S \in \mathbf{U}, T \in \mathbf{V}$ (with left unitary action whenever $T$ is a monoid) holds if and only if either $\mathbf{U} \subseteq \mathbf{L G}$ or $\mathbf{V} \subseteq \mathbf{G}$.

Proof. (i) Let $S \in \mathbf{L G}$ and let $T$ be regular. If $(s, t) \in S * T$, then, for $t^{\prime} \in V(t)$,

$$
(s, t)\left(t^{\prime} s^{-1}, t^{\prime}\right)(s, t)=\left(s^{t t^{\prime}}\left(s^{-1} s\right), t\right)=(s, t),
$$

since ${ }^{t t^{\prime}}\left(s^{-1} s\right)$ is an idempotent $\mathcal{L}$-related to $s$. Hence $S * T$ is regular.

(ii) Let $S$ be regular and let $T$ be a group acting left unitarily on $S$. Now any element $(s, t)$ of $S * T$ can be written as $\left(s s^{\prime}, 1\right)(s, t)$, where $s^{\prime} \in V(s)$. Since $\left(s s^{\prime}, 1\right)$ is an idempotent, the result follows from the remarks after Theorem 3.1.

Necessity entails an examination of various cases: if $\mathbf{U} \nsubseteq \mathbf{L} \mathbf{G}$ then it contains either a two element semilattice or a two element right zero semigroup; and if $\mathbf{V} \nsubseteq \mathbf{G}$ it contains either one of these or a two element left zero semigroup. The preceding theorem covers the situation where both $\mathbf{U}$ and $\mathbf{V}$ contain nontrivial semilattices. We consider the other combinations in turn.

(iii) Suppose $\mathbf{U}$ contains the semilattice $S=\{0,1\}, 0<1$, and $\mathbf{V}$ contains the two element semigroup $T=\{a, b\}$, either left or right zero. Consider $\alpha: T^{1} \rightarrow S$ defined by $a \alpha=b \alpha=0,1 \alpha=1$. For all $c \in T,{ }^{c} \alpha$ is the constant function $\zeta$ with value 0 . Thus for any $(\beta, d) \in S \mathrm{wr} T,(\alpha, a)(\beta, d)(\alpha, a)=\left(\alpha^{a} \beta^{a d} \alpha, a\right)=(\zeta, a)$, that is, $(\alpha, a)$ is not regular.

(iv) Suppose $\mathbf{U}$ contains the right zero semigroup $S=\{e, f\}$ and $\mathbf{V}$ contains the semilattice $T=\{0,1\}, 0<1$. Consider $\alpha: T^{1}=T \rightarrow S$ defined by $0 \alpha=e, 1 \alpha=f$. Then ${ }^{0} \alpha$ is the constant function $\zeta$ with value $e$. Thus for any $(\beta, d) \in S \mathrm{wr} T$, $(\alpha, 0)(\beta, d)(\alpha, 0)=(\zeta, 0)$, that is, $(\alpha, a)$ is not regular.

(v) Suppose $\mathbf{U}$ again contains the right zero semigroup $S=\{e, f\}$ and $\mathbf{V}$ contains a semigroup $T$ as in case (iii). Consider $\alpha: T^{1} \rightarrow S$ defined by $a \alpha=b \alpha=e$, $1 \alpha=f$. For any $(\beta, d) \in S \mathrm{wr} T,(\alpha, a)(\beta, d)(\alpha, a)=\left({ }^{a d} \alpha, a\right)$. If $T$ is right zero then $a d=d$; but ${ }^{d} \alpha$ is constant (with value $d \alpha$ ). If $T$ is left zero then $a d=a$; but $1\left({ }^{a} \alpha\right)=a \alpha \neq 1 \alpha$. In either case, $(\alpha, a)$ is not regular.

If the actions are not restricted as indicated then the second possibility in the theorem cannot occur. For, in the notation there, if $\mathbf{U}$ contains a nontrivial semilattice or right zero semigroup $S$, then for any trivial semigroup $T$ there is an action on $S$ such that the semidirect product is not regular.

Now that we have described precisely when the semidirect product $\mathbf{U} * \mathbf{V}$ of evarieties is well defined, we proceed to investigate its properties. First we note that by the remarks following the definition of wreath product an alternative formulation of the product exists. 
Proposition 3.3. Let $\mathbf{U}, \mathbf{V}$ be e-varieties of regular semigroups. Whenever $\mathbf{U} * \mathbf{V}$ is defined, it is generated by $\{\operatorname{Reg}(S \mathrm{wr} T): S \in \mathbf{U}, T \in \mathbf{V}\}$.

When considering varieties $\mathbf{U}, \mathbf{V}$ of semigroups in their usual sense, their semidirect product is that generated by all semidirect products $S * T, S \in \mathbf{U}, T \in \mathbf{V}$ (under the same restriction on actions), and the analogue of the previous proposition holds. In that situation, $\mathbf{U} * \mathbf{V}$ comprises simply the semigroups that divide (that is, are quotients of subsemigroups of) some semidirect product of this type. Although evarieties are in general not generated so straightforwardly, by Result 1.6 an analogue holds within $L \mathbf{I}$ and $\mathbf{E S}$.

It is well known that for any indexed families $\left(S_{i}\right)_{i \in I},\left(T_{i}\right)_{i \in I}$ of semigroups such that each $T_{i}$ acts on $S_{i}$, there is an action of $\prod T_{i}$ on $\prod S_{i}$ such that $\prod\left(S_{i} * T_{i}\right) \cong$ $\prod S_{i} * \prod T_{i}$. In the context of Theorem 3.1, then, the class $\{\operatorname{Reg}(S * T): S \in$ $\mathbf{U}, T \in \mathbf{V}\}$ is closed under products, when well defined. Making use of Result 1.6, we therefore have the following.

Proposition 3.4. Let $\mathbf{U}, \mathbf{V}$ be e-varieties of regular semigroups such that $\mathbf{U}$ or $\mathbf{V}$ is contained in $\mathbf{C S}$. If $\mathbf{U} * \mathbf{V}$ is contained within either $L \mathbf{I}$ or $\mathbf{E S}$, then it consists of all regular semigroups that regularly divide the set of regular elements of some semidirect product $S * T, S \in \mathbf{U}, T \in \mathbf{V}$.

On the one hand, therefore, when the semidirect product of e-varieties consists of locally inverse or of $E$-solid semigroups, it behaves amenably. We must consider the question of exactly when this occurs. On the other hand, one of the overall aims of this paper is to study exactly these e-varieties $L \mathbf{I}$ and ES and their sube-varieties, via semidirect products. The balance of this section is thus devoted, more generally, to discovering properties of $\operatorname{Reg}(S * T)$ that follow from those of $S$ and $T$. The next sections will treat the converse aspect: decomposing e-varieties as semidirect products of sub-e-varieties.

We first investigate the local monoids and cores of semidirect products.

Proposition 3.5. If $S$ and $T$ are arbitrary semigroups, with $T$ acting on $S$, then each local monoid of $S * T$ embeds in a semidirect product of a subsemigroup of $S$ with a local monoid of $T$, with left unitary action. If, further, $S$ and $T$ are regular, then the designated subsemigroups of $S$ and $T$ are regular.

Hence, if $\mathbf{U}$ and $\mathbf{W}$ are e-varieties, then $\mathbf{U} * L \mathbf{W} \subseteq L(\mathbf{U} * \mathbf{W})$ whenever $\mathbf{U} \subseteq \mathbf{C S}$ or $\mathbf{W} \subseteq \mathbf{C S}$.

Proof. Let $(x, e) \in E_{S * T}$. Then $e T e$ is a local submonoid of $T$ (and so is regular if $T$ is). Also ${ }^{e} S\left(=\left\{{ }^{e} x: x \in S\right\}\right)$ is a subsemigroup of $S$ (and is regular, if $S$ is, since it is the image of $S$ under the endomorphism induced by $e$ ); and the action of $T$ on $S$ restricts to a left unitary action of $e T e$ on ${ }^{e} S$. Define $\theta:(x, e)(S * T)(x, e) \rightarrow$ ${ }^{e} S * e T e$ by

$$
(y, t) \theta=\left({ }^{e} y, t\right), \text { for }(y, t) \in(x, e)(S * T)(x, e) .
$$

For such a pair $(y, t),(y, t)=(x, e)(y, t)=(y, t)(x, e)$, so that $t=e t=t e \in e T e$ and $y=x^{e} y=y^{t} x$. Now let $(y, t),(z, u) \in(x, e)(S * T)(x, e)$. Then

$$
\begin{aligned}
{[(y, t)(z, u)] \theta } & =\left(y^{t} z, t u\right) \theta=\left({ }^{e}\left(y^{t} z\right), t u\right) \\
& =\left({ }^{e} y^{t} z, t u\right)=\left({ }^{e} y, t\right)\left({ }^{e} z, u\right)=(y, t) \theta(z, u) \theta .
\end{aligned}
$$

If $(y, t) \theta=(z, u) \theta$, then ${ }^{e} y={ }^{e} z$ and $t=u$. Since $y=x^{e} y$ and $z=x^{e} z$, we have $y=z$. Hence $\theta$ is an embedding of $(x, e)(S * T)(x, e)$ in ${ }^{e} S * e T e$, which restricts to an embedding of $(x, e) \operatorname{Reg}(S * T)(x, e)$, if $\operatorname{Reg}(S * T) \leq S * T$. 
Applying Theorem 3.1, and using the equation $\mathbf{C S}=L \mathbf{C S}$, the final sentences follow from the left unitariness of the action, which guarantees that the semidirect product belongs to the appropriate product of e-varieties.

Some particular cases, based on the equations $\mathbf{R B}=L \mathbf{T}$ and $\mathbf{C S}=L \mathbf{G}$, are worthy of note.

Corollary 3.6. The following inclusions hold for an e-variety $\mathbf{U}$ :

(i) $\mathbf{U} * \mathbf{R B} \subseteq L \mathbf{U}$ for any $\mathbf{U}$;

(ii) $\mathbf{U} * \mathbf{C S} \subseteq L(\mathbf{U} * \mathbf{G})$ for any $\mathbf{U}$;

(iii) $\mathbf{U} * L \mathbf{I} \subseteq L(\mathbf{U} * \mathbf{I})$, for $\mathbf{U} \subseteq \mathbf{C S}$;

(iv) $\mathbf{U} * L \mathbf{E S} \subseteq L(\mathbf{U} * \mathbf{E S})$, for $\mathbf{U} \subseteq \mathbf{C S}$.

Corollary 3.7. Let $S$ be a rectangular band and $T$ any semigroup acting on $S$. Then each local monoid of $S * T$ embeds in a local monoid of $T$. Hence for any e-variety $\mathbf{W}, \mathbf{R B} * L \mathbf{W} \subseteq L \mathbf{W}$.

Proof. In the notation of the proposition, if $S$ is a rectangular band, then for any $(y, t) \in(x, e)(S * T)(x, e)$ we have $y=x^{t} x$. Hence the restriction of $\theta$ to the second projection embeds $(x, e)(S * T)(x, e)$ in $e T e$.

We turn now to the core and self-conjugate core. A useful, easily verified observation is the following: if $S$ and $T$ are regular semigroups such that $\operatorname{Reg}(S * T) \leq S * T$, then $\operatorname{Reg}(S * U) \leq S * U$ for any regular subsemigroup $U$ of $T$ that includes all inverses of its elements.

Proposition 3.8. Let $S$ and $T$ be regular semigroups, $T$ acting on $S$, such that $\operatorname{Reg}(S * T) \leq S * T$. Then $C(S * T)$ is a regular subsemigroup of $\operatorname{Reg}(S * C(T))$ and $C_{\infty}(S * T)$ is a regular subsemigroup of $\operatorname{Reg}\left(S * C_{\infty}(T)\right)$.

Hence, if $\mathbf{U}$ and $\mathbf{W}$ are e-varieties, then $\mathbf{U} * C \mathbf{W} \subseteq C(\mathbf{U} * \mathbf{W})$ and $\mathbf{U} * C_{\infty} \mathbf{W} \subseteq$ $C_{\infty}(\mathbf{U} * \mathbf{W})$, whenever $\mathbf{U} \subseteq \mathbf{C S}$ or $\mathbf{W} \subseteq \mathbf{C S}$.

Proof. Denote by $\pi_{R}$ the restriction to $\operatorname{Reg}(S * T)$ of the projection $S * T \rightarrow$ $T$. Note that $\left({ }^{t t^{\prime}} f, t\right) \in \operatorname{Reg}(S * T)$ for any $t \in T, t^{\prime} \in V(t)$ and $f \in E_{S}$, so $\pi_{R}$ is surjective. Since $C(\operatorname{Reg}(S * T)) \pi_{R} \subseteq C(T), C(\operatorname{Reg}(S * T))$ is a (regular) subsemigroup of $S * C(T)$. By [38, Lemma 2.3], $C(T)$ includes all inverses of its elements, so $\operatorname{Reg}(S * C(T)) \leq S * C(T)$. Hence $C(\operatorname{Reg}(S * T))$ is a regular subsemigroup of $\operatorname{Reg}(S * C(T))$.

A similar argument applies to $C_{\infty}(S * T)$. The final statement follows by applying Theorem 3.1, using the equation $\mathbf{C S}=C \mathbf{C S}=C_{\infty} \mathbf{C S}$. (Note that $T \in C \mathbf{W}$ acts monoidally on $S$ if and only if the same is true for $C(T) \in \mathbf{W}$, and similarly for $C_{\infty} \mathbf{W}$.)

Again, some particular cases, based on the equations $\mathbf{G}=C_{\infty} \mathbf{T}=C \mathbf{T}, \mathbf{I}=$ $C_{\infty} \mathbf{S}=C \mathbf{S}, \mathbf{O}=C_{\infty} \mathbf{B}=C \mathbf{B}$ and $\mathbf{E S}=C_{\infty} \mathbf{C R}=C \mathbf{C R}$, are worthy of note.

Corollary 3.9. The following inclusions hold for an e-variety $\mathbf{U}$ :

(i) $\mathbf{U} * \mathbf{G} \subseteq C_{\infty} \mathbf{U} \subseteq C \mathbf{U}$, for any $\mathbf{U}$;

(ii) in particular, $\mathbf{C R}(\mathbf{H}) * \mathbf{G} \subseteq C_{\infty} \mathbf{C R}(\mathbf{H}) \subseteq \mathbf{E S}$ for all group varieties $\mathbf{H}$;

(iii) $\mathbf{U} * \mathbf{I} \subseteq C_{\infty}(\mathbf{U} * \mathbf{S})$ for all $\mathbf{U} \subseteq \mathbf{C S}$;

(iv) $\mathbf{U} * \mathbf{O} \subseteq C_{\infty}(\mathbf{U} * \mathbf{B})$ for all $\mathbf{U} \subseteq \mathbf{C S}$;

(v) $\mathbf{U} * \mathbf{E S} \subseteq C_{\infty}(\mathbf{U} * \mathbf{C R})$ for all $\mathbf{U} \subseteq \mathbf{C S}$. 
As remarked in $\S 1$, in general $C_{\infty} \mathbf{U}$ is properly contained in $C \mathbf{U}$. By combining the above results we obtain some useful equalities.

Proposition 3.10. Let $\mathbf{U}$ be any e-variety of the form $L C \mathbf{W}$, for some e-variety W. Then for any $S \in \mathbf{U}$ and any completely simple semigroup $T$ acting on $S$, $\operatorname{Reg}(S * T) \in \mathbf{U}$. Hence $\mathbf{U} * \mathbf{C S}=\mathbf{U}$. In particular,

(i) $\mathbf{C S} * \mathbf{C S}=\mathbf{C S}$;

(ii) $L \mathbf{I} * \mathbf{C S}=L \mathbf{I}$;

(iii) $L \mathbf{O} * \mathbf{C S}=L \mathbf{O}$;

(iv) $L \mathbf{E S} * \mathbf{C S}=L \mathbf{E S}$.

Proof. Let $\mathbf{U}$ be such an e-variety. By Corollaries 3.6(ii) and 3.9(i), $\mathbf{U} * \mathbf{C S} \subseteq L(\mathbf{U} *$ $\mathbf{G}) \subseteq L C \mathbf{U}$. But by Proposition 1.3, LCU $=L C L C \mathbf{W} \subseteq L L C C \mathbf{W}=L C \mathbf{W}=\mathbf{U}$. The particular cases follow from the equations given earlier. The statement about individual semigroups (where the action need not be left unitary, even if $T$ is a monoid) follows from the corresponding parts of the relevant earlier results.

Now we answer the question of when $\mathbf{U} * \mathbf{V}$ lies within $L \mathbf{I}$ or $\mathbf{E S}$, in the case where the second factor is completely simple.

Proposition 3.11. Let $\mathbf{U}, \mathbf{V}$ be e-varieties of regular semigroups, with $\mathbf{V} \subseteq \mathbf{C S}$. Then

(a) $\mathbf{U} * \mathbf{V} \subseteq L \mathbf{I}$ if and only if $\mathbf{U} \subseteq L \mathbf{I}$, and

(b) $\mathbf{U} * \mathbf{V} \subseteq \mathbf{E S}$ if and only if either $\mathbf{U} \subseteq \mathbf{C S}$ or both $\mathbf{U} \subseteq \mathbf{E S}$ and $\mathbf{V} \subseteq \mathbf{L G}$.

Proof. (a) Sufficiency is immediate from (ii) of the preceding proposition. Necessity is clear.

(b) If $\mathbf{U} \subseteq \mathbf{C S}$, then $\mathbf{U} * \mathbf{V} \subseteq \mathbf{C S} \subseteq \mathbf{E S}$, by (i) of the preceding proposition.

Next suppose $S \in \mathbf{E S}$ and $T \in \mathbf{L G}$. Let $(x, e),(y, f) \in E_{S * T}$, so that $e, f \in E_{T}$ (whence $e f=e$ ), $x^{e} x=x$ and $y^{f} y=y$. Then ${ }^{e} x$ and ${ }^{e} y$ are idempotents of $S$, so their product ${ }^{e}(x y)$ belongs to a subgroup. Hence there exists $u \in S$ such that $u\left({ }^{e}(x y)\right)^{2}=\left({ }^{e}(x y)\right)^{2} u={ }^{e}(x y)$. We show that the product $(x, e)(y, f)=$ $\left(x^{e} y, e\right)$ is $\mathcal{H}$-related to its square, and so belongs to a subgroup. Note that $x^{e} y=x^{e}(x y)$. Then $(x u, e)\left(x^{e} y, e\right)^{2}=\left(x u^{e}(x y)^{e}(x y), e\right)=\left(x^{e}(x y), e\right)=\left(x^{e} y, e\right)$ and $\left(x^{e} y, e\right)^{2}(u, e)=\left(x^{e}(x y)^{e}(x y)^{e} u, e\right)=\left(x^{e}\left({ }^{e}(x y)^{e}(x y) u\right), e\right)=\left(x^{e}(x y), e\right)=$ $\left(x^{e} y, e\right)$, as required. Thus $\operatorname{Reg}(S * T) \in \mathbf{E S}$, completing the proof of sufficiency.

Conversely, $\mathbf{U} \subseteq \mathbf{E S}$ is clearly necessary. If $\mathbf{U} \nsubseteq \mathbf{C S}$ and $\mathbf{V} \nsubseteq \mathbf{L G}$ then $\mathbf{U}$ contains the semilattice $S=\{0,1\}$ and $\mathbf{V}$ the two element right zero semigroup $T=\{e, f\}$. In $S$ wr $T$, an element $(\alpha, c)$ is idempotent if and only if ${ }^{c} \alpha \geq \alpha$ in $S^{T^{1}}$. Let $\alpha, \beta: T^{1} \rightarrow S$ be defined by $e \alpha=0, f \alpha=1 \alpha=1$ and $e \beta=f \beta=1 \beta=1$. Then $(\alpha, f),(\beta, e) \in E_{S \mathrm{wr} T}$, but $(\alpha, f)(\beta, e)=\left(\alpha^{f} \beta, e\right)$, where $f\left(\alpha^{f} \beta\right)=f \alpha f \beta=1$ while $f^{e}\left(\alpha^{f} \beta\right)=e \alpha f \beta=0$; that is, the product is nonidempotent. Since it is well known that a wreath product of aperiodic semigroups is aperiodic, the product in fact lies in no subgroup of $S$ wr $T$. Hence $S$ wr $T \notin \mathbf{E S}$.

(In Proposition 4.6 we shall see that $\mathbf{S} * \mathbf{R Z}=\mathbf{C S R}$, the e-variety of combinatorial strict regular semigroups. With very little extra work, the example above indeed proves this fact.)

Before considering the alternative situation, we need one further preliminary result. 
Lemma 3.12. If $S$ is completely simple and $T$ is completely regular, then $\operatorname{Reg}(S * T)$ is completely regular. Thus $\mathbf{C S} * \mathbf{C R}=\mathbf{C R}$.

Proof. We may write $T=\bigcup_{\alpha \in A} T_{\alpha}$, where $\left(T_{\alpha}\right)_{\alpha \in A}$ are its $\mathcal{D}$-classes. Then $S * T=$ $\bigcup_{\alpha \in A}\left(S * T_{\alpha}\right)$ and $\operatorname{Reg}(S * T)=\bigcup_{\alpha \in A} \operatorname{Reg}\left(S * T_{\alpha}\right)$. Since each $T_{\alpha}$ is completely simple, by Proposition 3.10 so is each term in the last union, whence $\operatorname{Reg}(S * T) \in$ CR.

Corollary 3.13. The following equalities hold:

(i) $\mathbf{C S} * \mathbf{E S}=\mathbf{E S}$;

(ii) $\mathbf{C S} * L \mathbf{E S}=L \mathbf{E S}$.

Proof. From Corollary 3.9 and Lemma 3.12 we obtain $\mathbf{C S} * \mathbf{E S} \subseteq C(\mathbf{C S} * \mathbf{C R}) \subseteq$ $C \mathbf{C R}=\mathbf{E S}$. The second statement follows from Corollary 3.6(iv).

Now we can answer the question posed earlier in the case when the first factor is completely simple.

Proposition 3.14. Let $\mathbf{U}, \mathbf{V}$ be e-varieties of regular semigroups, with $\mathbf{U} \subseteq \mathbf{C S}$. Then

(a) $\mathbf{U} * \mathbf{V} \subseteq L \mathbf{I}$ if and only if either $\mathbf{V} \subseteq \mathbf{C S}$ or both $\mathbf{U} \subseteq \mathbf{R B}$ and $\mathbf{V} \subseteq L \mathbf{I}$, and

(b) $\mathbf{U} * \mathbf{V} \subseteq \mathbf{E S}$ if and only if $\mathbf{V} \subseteq \mathbf{E S}$.

Proof. (a) Sufficiency follows from Proposition 3.10(i) and Corollary 3.7.

Conversely, $\mathbf{V} \subseteq L \mathbf{I}$ is clearly necessary. If $\mathbf{U} \nsubseteq \mathbf{R B}$ and $\mathbf{V} \nsubseteq \mathbf{C S}$, then $\mathbf{U}$ contains a nontrivial group $G$, with identity element $e$ and nonidentity element $g$, say, and $\mathbf{V}$ contains the semilattice $T=\{0,1\}, 0<1$. Let $\alpha, \beta: T \rightarrow G$ be given by $0 \alpha=e, 1 \alpha=g$ and $0 \beta=1 \beta=e$. Then $(\alpha, 0),(\beta, 0)$ are easily verified to be $\mathcal{L}$-related idempotents in $G \mathrm{wr} T$. Since this wreath product is a monoid, it does not belong to $L \mathbf{I}$.

(b) Sufficiency was proved in Corollary 3.13, and necessity is clear.

\section{Decompositions of The FORM $-* \mathbf{R Z}$}

Our goal in this section is to use McAlister's matrix representation theorems for classes such as $L \mathbf{I}, L \mathbf{O}, L \mathbf{E S}$, in conjunction with Corollary 3.6(i), to obtain the decompositions $L \mathbf{I}=\mathbf{I} * \mathbf{R Z}, L \mathbf{O}=\mathbf{O} * \mathbf{R Z}$ and $L \mathbf{E S}=\mathbf{E S} * \mathbf{R Z}$; refinements of McAlister's theorems will lead to similar decompositions for various sub-e-varieties. The decomposition of $L \mathbf{I}$ will be used in $\S 8$ to describe the e-free locally inverse semigroups. (Since $L \mathbf{O}$, and therefore $L \mathbf{E S}$, is contained in neither $L \mathbf{I}$ nor ES, e-free semigroups of rank greater than one do not exist there, by Result 1.4.)

The Rees matrix semigroup $\mathcal{M}(I, S, \Lambda, P)$, where, for the moment, $S$ is an arbitrary semigroup, $I, \Lambda$ are nonempty sets and $P$ is a $\Lambda \times I$ matrix over $S$, has as its underlying set $I \times S \times \Lambda$, with product $(i, s, \lambda)(j, t, \mu)=\left(i, s p_{\lambda j} t, \mu\right)$, in the usual manner. The following result is a refinement of [6, Proposition XI.3.1].

Proposition 4.1. Any Rees matrix semigroup $\mathcal{M}(I, S, \Lambda, P)$ embeds in $(S \mathrm{wr} \Lambda) \times$ $I$, where $\Lambda$ and $I$ are endowed with right zero and left zero multiplication, respectively.

Proof. Define $\phi: \mathcal{M}(I, S, \Lambda, P) \rightarrow(S$ wr $\Lambda) \times I$ by

$$
(i, s, \lambda) \phi=\left(\left(f_{(i, s, \lambda)}, \lambda\right), i\right),
$$


where $f_{(i, s, \lambda)}: \Lambda^{1} \rightarrow S$ is given by $\mu f_{(i, s, \lambda)}=p_{\mu i} s$, if $\mu \in \Lambda$, and $1 f_{(i, s, \lambda)}=s$. It is routinely verified that $\phi$ is a morphism. Suppose that $(i, s, \lambda) \phi=\left(i^{\prime}, s^{\prime}, \lambda^{\prime}\right) \phi$. Then $i=i^{\prime}, \lambda=\lambda^{\prime}$ and $1 f_{(i, s, \lambda)}=1 f_{\left(i^{\prime}, s^{\prime}, \lambda^{\prime}\right)}$, whence $s=s^{\prime}$. Hence $\phi$ is an embedding.

McAlister [23] showed that if $S$ is a regular semigroup then the regular elements of $\mathcal{M}(I, S, \Lambda, P)$ form a subsemigroup, which he called a regular Rees matrix semigroup and denoted $\mathcal{R} \mathcal{M}(I, S, \Lambda, P)$. We prefer the notation $\operatorname{Reg} \mathcal{M}(I, S, \Lambda, P)$. It follows immediately from the proposition that if $S$ belongs to some e-variety $\mathbf{U}$, then $\operatorname{Reg} \mathcal{M}(I, S, \Lambda, P) \in(\mathbf{U} * \mathbf{R Z}) \vee \mathbf{L Z}$. The inclusion can be clarified by the following result, which fails when $\mathbf{U}=\mathbf{T}$ or $\mathbf{U}=\mathbf{R Z}$, since the wreath product of $\mathcal{L}$-trivial semigroups is again $\mathcal{L}$-trivial.

Lemma 4.2. For any e-variety $\mathbf{U}$ that is not contained in $\mathbf{R Z}, \mathbf{L Z} \subseteq \mathbf{U} * \mathbf{R Z}$.

Proof. Since $\mathbf{U} \nsubseteq \mathbf{R Z}, \mathbf{U}$ contains a nontrivial semigroup that is either left zero, a group, or a semilattice. In the first case, the result clearly holds. In the other cases $\mathbf{U}$ contains a nontrivial monoid $M$, say, with identity 1 . Let $T=\{a, b\}$ be a two-element right zero semigroup. Now for any $\alpha: T^{1} \rightarrow M$ such that $a \alpha=1,{ }^{a} \alpha$ is the identity of $M^{T^{1}}$, so that the set of all such pairs $(\alpha, a)$ consists of $\mathcal{L}$-related idempotents. Since there are at least two such choices of $\alpha$, the wreath product contains a two-element left zero subsemigroup.

Corollary 4.3. Let $\mathbf{U}$ be an e-variety that is not contained in $\mathbf{R Z}$. Then for any $S \in \mathbf{U}, \operatorname{Reg} \mathcal{M}(I, S, \Lambda, P) \in \mathbf{U} * \mathbf{R Z}$.

Now we state the theorem of McAlister to be used in the sequel, in its most general form [25] (originally proved for locally inverse semigroups in [24]). A morphism $S \rightarrow T$ of regular semigroups is a local isomorphism if it is injective on the local monoids of $S$.

Result 4.4. Let $\mathbf{U}$ be any one of $\mathbf{I}, \mathbf{O}$ or $\mathbf{E S}$. Then a regular semigroup belongs to $L \mathbf{U}$ if and only if it is a locally isomorphic image of a regular Rees matrix semigroup over $\mathbf{U}$.

McAlister proves, as well, that the result is true for $\mathbf{U}$ the e-variety of $\mathcal{L}$-unipotent regular semigroups (and its dual), but we shall concentrate on the cases listed. Of course, by Rees' Theorem, the result holds for $\mathbf{U}=\mathbf{G}$ as well. McAlister also shows that the result is not true when $\mathbf{U}$ is the (e-) variety $\mathbf{C R}$ or $\mathbf{S}$ (or, similarly, $\mathbf{S G}$ ).

In conjunction with Corollary 3.6(i), from the previous corollary, result and remarks we immediately obtain our first decompositions.

Theorem 4.5. The following equations hold: $\mathbf{C S}=\mathbf{G} * \mathbf{R Z}, L \mathbf{I}=\mathbf{I} * \mathbf{R Z}, L \mathbf{O}=$ $\mathbf{O} * \mathbf{R Z}, L \mathbf{E S}=\mathbf{E S} * \mathbf{R Z}$.

It is in fact true that the analogue of this theorem is valid for $L \mathbf{S G}$ ( $=\mathbf{S R}$ ) and for $L \mathbf{S}$ ( = CSR), although the remarks following the statement of McAlister's result make it clear that a different proof is required:

Proposition 4.6. For every variety $\mathbf{U}$ of Clifford semigroups, $L \mathbf{U}=\mathbf{U} * \mathbf{R Z}$. In particular, $\mathbf{S R}=\mathbf{S G} * \mathbf{R Z}$ and $\mathbf{C S R}=\mathbf{S} * \mathbf{R Z}$.

Proof. It is known (see [31]) that $\mathbf{U}$ has the form $\mathbf{S H}$, comprising all semilattices of groups from some group variety $\mathbf{H}$. Now any member of $L \mathbf{U}$ is a strict regular semigroup and is therefore isomorphic to a subdirect product of its principal factors, 
which are completely simple or completely 0 -simple semigroups with subgroups from $\mathbf{H}$. Any completely simple factor belongs to $\mathbf{H} * \mathbf{R Z}$, by Theorem 4.5. Any completely 0-simple factor is isomorphic to a (regular) Rees matrix semigroup with zero $\mathcal{M}^{0}(I, H, \Lambda, P)$, for some group $H \in \mathbf{H}$. Now this semigroup is in turn isomorphic with the Rees quotient $\mathcal{M}\left(I, H^{0}, \Lambda, P\right) / K$, where $K$ is the ideal $I \times\{0\} \times \Lambda$, and by Corollary 4.3 therefore belongs to $\mathbf{S H} * \mathbf{R Z}$.

We do not know whether $L \mathbf{C R}=\mathbf{C R} * \mathbf{R Z}$ or, indeed, whether any example exists for which the analogue of Theorem 4.5 fails.

The remainder of the section is devoted to refining Theorem 4.5 so as to encompass sub-e-varieties of $L \mathbf{E S}$, by means of a refinement of McAlister's result. We shall first state the result, its consequences and some related conclusions, and then proceed to its proof. Recall that a subsemigroup $Q$ of a semigroup $S$ is a quasi-ideal if $Q S Q \subseteq Q$. If $S$ and $Q$ are regular then $Q$ is a regular quasi-ideal, in which case it is clear that $Q=Q S Q$.

Theorem 4.7. Let $S$ be a regular semigroup and $\operatorname{Reg} \mathcal{M}(I, S, \Lambda, P)$ a regular Rees matrix semigroup over $S$. Then $S$ contains a regular quasi-ideal $Q$ such that

(i) $\operatorname{Reg} \mathcal{M}(I, S, \Lambda, P)=\operatorname{Reg} \mathcal{M}(I, Q, \Lambda, P)=R$, say, and

(ii) $R$ and $Q$ have the same local monoids, up to isomorphism.

As a consequence we obtain the following refinement of Theorem 4.5.

Theorem 4.8. Let $\mathbf{C}$ be any one of $\mathbf{G}, \mathbf{I}, \mathbf{O}$ or $\mathbf{E S}$ and let $\mathbf{W}$ be a sub-e-variety of $\mathbf{C}$. Then $L \mathbf{W}=(L \mathbf{W} \cap \mathbf{C}) * \mathbf{R Z}$, except in the case $\mathbf{C} \subseteq \mathbf{I}$ and $\mathbf{W}=\mathbf{T}$.

Proof. Follow the same argument as for Theorem 4.5, combining the theorem with McAlister's result. The exclusion corresponds to the case $L \mathbf{W} \cap \mathbf{I} \subseteq \mathbf{R Z}$ excluded from Lemma 4.2.

Corollary 4.9. The following equations hold:

(i) $L \mathbf{C R}=(L \mathbf{C R} \cap \mathbf{E S}) * \mathbf{R Z}$,

(ii) $\mathbf{S R}=\mathbf{S I} * \mathbf{R Z}$ and

(iii) $\mathbf{C S R}=\mathbf{C S I} * \mathbf{R Z}$;

(iv) $\mathbf{C S}(\mathbf{H})=\mathbf{H} * \mathbf{R Z}$ for any nontrivial group variety $\mathbf{H}$.

Proof. The first equation is clear. The second follows from the equations $\mathbf{S R}=$ $L \mathbf{S G}$ and $\mathbf{S I}=\mathbf{S R} \cap \mathbf{I}$, and the third similarly. For the fourth equation, let $\mathbf{C}=\mathbf{G}$ and $\mathbf{W}=\mathbf{H}$ and use the fact that $\mathbf{C S}(\mathbf{H})=L \mathbf{H}$.

The second and third equations in the corollary are also consequences of Proposition 4.6.

The rest of the section is devoted to the proof of Theorem 4.7. We begin with some generalities on regular semigroups.

Proposition 4.10. Let $S$ be a regular semigroup. Then

(i) for any subset $A$ of $S$ such that $A \cap V(a) \neq \emptyset$ for each $a \in A$, ASA is a regular quasi-ideal of $S$ (and every regular quasi-ideal arises in this way);

(ii) for any regular quasi-ideal $Q_{0}$ of $S$, the set $Q=\left\{s \in S: V(s) \cap Q_{0} \neq \emptyset\right\}$ is a regular quasi-ideal of $S$ containing $Q_{0}$.

Proof. (i) Clearly $A S A$ is a quasi-ideal. Let $a, b \in A$, with inverses $a^{\prime}, b^{\prime}$ respectively, in $A$, and let $s \in S$. By Lemma $1.2, V(a s b) \cap b^{\prime} S a^{\prime} \neq \emptyset$, so that $V(a s b) \cap A S A \neq \emptyset$. The parenthetical remark is clear, since $\mathrm{Q}=\mathrm{QSQ}$ for any regular quasi-ideal. 
(ii) Let $a, b \in Q$, with inverses $a^{\prime}, b^{\prime} \in Q_{0}$, and let $s \in S$. As in (i), $V(a s b) \cap$ $Q_{0} S Q_{0} \neq \emptyset$, so that $a s b \in Q$. Hence $Q$ is a quasi-ideal. Since $Q_{0}$ is regular, $Q_{0} \subseteq Q$ and $Q$ is regular.

We also need some elementary properties of regular Rees matrix semigroups.

Result 4.11 ([23]). Let $\operatorname{Reg} \mathcal{M}(I, S, \Lambda, P)$ be a regular Rees matrix semigroup over the (regular) semigroup $S$. Then

(i) $\operatorname{Reg} \mathcal{M}(I, S, \Lambda, P)=\left\{(i, s, \lambda): V(s) \cap p_{\lambda j} S p_{\mu i} \neq \emptyset\right.$ for some $\left.j \in I, \mu \in \Lambda\right\}$;

(ii) $(i, s, \lambda)$ is idempotent if and only if $s=s p_{\lambda i} s$.

Part (i) of the theorem under consideration is covered by the following.

Proposition 4.12. Consider $\mathcal{M}(I, S, \Lambda, P)$, where $S$ is regular. Treating $P$ as a subset of $S$, put $P^{\prime}=\bigcup_{p \in P} V(p)$; let $Q_{0}=\left(P \cup P^{\prime}\right) S\left(P \cup P^{\prime}\right)$ and $Q=\{s \in S$ : $\left.V(s) \cap Q_{0} \neq \emptyset\right\}$. Then $Q$ is a regular quasi-ideal of $S$ such that $\operatorname{Reg} \mathcal{M}(I, S, \Lambda, P)=$ $\operatorname{Reg} \mathcal{M}(I, Q, \Lambda, P)$.

Proof. That $Q$ is a regular quasi-ideal is immediate from the preceding proposition. Clearly $Q$ contains $P$, so $\operatorname{Reg} \mathcal{M}(I, Q, \Lambda, P)$ is well defined and is a subset of $\operatorname{Reg} \mathcal{M}(I, S, \Lambda, P)$. Conversely, if $s \in S$ and $(i, s, \lambda) \in \operatorname{Reg} \mathcal{M}(I, S, \Lambda, P)$, then by Result $4.11 V(s) \cap p_{\lambda j} S p_{\mu i} \neq \emptyset$, for some $j \in I, \mu \in \Lambda$, so $V(s) \cap Q_{0} \neq \emptyset$, that is, $s \in Q$. Hence the reverse inclusion holds.

Put $R=\operatorname{Reg} \mathcal{M}(I, S, \Lambda, P)=\operatorname{Reg} \mathcal{M}(I, Q, \Lambda, P)$. That each local submonoid of $R$ is isomorphic to a local submonoid of $Q$ is the content of [25, Lemma 2.6(i)]. To prove the converse, let $e$ be any idempotent of $Q$, so that $e$ has an inverse $e^{\prime}$ in $Q_{0}$. Since $Q_{0} \subseteq P S \cup P^{\prime} S$, the proof breaks into two cases: (a) $e^{\prime} \in P S$, (b) $e^{\prime} \in P^{\prime} S$.

In case (a), $e^{\prime} \in p_{\lambda i} S$ for some $i \in I, \lambda \in \Lambda$. Let $p_{\lambda i}^{\prime} \in V\left(p_{\lambda i}\right)$. We will prove that the map $\theta$, defined by $x \theta=\left(i, p_{\lambda i}^{\prime} e^{\prime} x, \lambda\right), x \in e Q e$, is an isomorphism of $e Q e$ with $f R f$, where $f=\left(i, p_{\lambda i}^{\prime} e^{\prime} e, \lambda\right)$ is an idempotent of $R$. Since $p_{\lambda i}^{\prime} e^{\prime} x=p_{\lambda i}^{\prime} e^{\prime} x e$, by Lemma $1.2, V\left(p_{\lambda i}^{\prime} e^{\prime} x\right) \cap e^{\prime} S p_{\lambda i} \neq \emptyset$, and so $V\left(p_{\lambda i}^{\prime} e^{\prime} x\right) \cap p_{\lambda i} S p_{\lambda i} \neq \emptyset$, whence $x \theta \in R$, by the preceding result.

Now let $x, y \in e Q e$. Note that $x e^{\prime} y=x e e^{\prime} e y=x e y=x y$. We have

$$
\left(i, p_{\lambda i}^{\prime} e^{\prime} x, \lambda\right)\left(i, p_{\lambda i}^{\prime} e^{\prime} y, \lambda\right)=\left(i, p_{\lambda i}^{\prime} e^{\prime} x p_{\lambda i} p_{\lambda i}^{\prime} e^{\prime} y, \lambda\right)=\left(i, p_{\lambda i}^{\prime} e^{\prime} x y, \lambda\right)
$$

using the above equation and the fact that $e^{\prime} \in p_{\lambda i} S$, (so that $p_{\lambda i} p_{\lambda i}^{\prime} e^{\prime}=e^{\prime}$ ). Hence $\theta$ is a morphism, $f$ is an idempotent and $(e Q e) \theta \subseteq f R f$. Further, if $\left(i, p_{\lambda i}^{\prime} e^{\prime} x, \lambda\right)=$ $\left(i, p_{\lambda i}^{\prime} e^{\prime} y, \lambda\right)$, then $x=e p_{\lambda i} p_{\lambda i}^{\prime} e^{\prime} x=e p_{\lambda i} p_{\lambda i}^{\prime} e^{\prime} y=y$, similarly.

Finally, let $(j, y, \mu) \in f R f$, with $y \in Q$. Then $j=i, \mu=\lambda$ and $y=$ $p_{\lambda i}^{\prime} e^{\prime} e p_{\lambda j} y p_{\mu i} p_{\lambda i}^{\prime} e^{\prime} e$. Since $e \in Q, q=e p_{\lambda i} y \in e Q e$. Again, since $e^{\prime} \in p_{\lambda i} S$, $p_{\lambda i}^{\prime} e^{\prime} e p_{\lambda i}$ is an idempotent, so $p_{\lambda i}^{\prime} e^{\prime} q=p_{\lambda i}^{\prime} e^{\prime} e p_{\lambda i} y=y$, that is, $q \theta=(j, y, \mu)$.

In case (b), $e^{\prime} \in p_{\lambda i}^{\prime} S$, for some $p_{\lambda i}^{\prime} \in V\left(p_{\lambda i}\right)$. With the notation as in (a), a similar analysis shows that the map $x \rightarrow\left(i, e^{\prime} x p_{\lambda i}^{\prime}, \lambda\right)$ is an isomorphism of $e Q e$ on $g R g$, where $g=\left(i, e^{\prime} e p_{\lambda i}^{\prime}, \lambda\right) \in E_{R}$.

This completes the proof of Theorem 4.7.

\section{Decompositions INVOLVING GROUPS}

5.1. Decompositions of the form $-*$ G. We consider, rather more generally, characterizations of semidirect products $\mathbf{U} * \mathbf{K}$, where $\mathbf{U}$ is any e-variety of $E$-solid semigroups and $\mathbf{K}$ is any variety of groups. When $\mathbf{U}$ is e-local we show that the semidirect product and the Mal'cev product coincide. In the case $\mathbf{K}=\mathbf{G}$, these also 
coincide with $C_{\infty} \mathbf{U}$, by Corollary 1.9. In particular, we obtain the decompositions $C_{\infty} \mathbf{C R}(\mathbf{H})=\mathbf{C R}(\mathbf{H}) * \mathbf{G}$, for any group variety $\mathbf{H}$. Most important, perhaps, is the decomposition $\mathbf{E S}=\mathbf{C R} * \mathbf{G}$. This particular equation was obtained by Szendrei [35] as a consequence of her description of the e-free $E$-solid semigroups; in the sequel we shall, to the contrary, derive such a description and, more generally, a description of the e-free semigroups in any semidirect product $\mathbf{U} * \mathbf{K}$, as above, from these equations.

The key to obtaining these equations is to apply Result 2.4 on the e-locality of $\mathbf{C R}(\mathbf{H})$, in conjunction with a 'regular' version of the 'Derived Semigroupoid Theorem' of Tilson [37, Theorem B.1]. We shall not go into this topic in full generality; nevertheless our presentation will be self-contained. A similar argument will be used in the next subsection. Further use of graphical methods will be seen in $\S \S 7.2$ and 7.3 .

The analogue of the first part of the following proposition in the varietal and pseudovarietal settings is well known.

Proposition 5.1. Let $\mathbf{U}$ be an e-local e-variety of E-solid regular semigroups and $\mathbf{K}$ a variety of groups. Then $\mathbf{U} * \mathbf{K}=\mathbf{U m K}$.

Hence equality holds for $\mathbf{U}=\mathbf{C R}(\mathbf{H})$, for any group variety $\mathbf{H}$ (in particular for $\mathbf{C R}$ itself) and for $\mathbf{U}$ any variety of bands.

Proof. The inclusion $\mathbf{U} * \mathbf{K} \subseteq \mathbf{U m K}$ follows from the observation that for any semidirect product $U * V$, with $U \in \mathbf{U}$ and $V \in \mathbf{K}, 1 \pi^{-1}$ is a regular subsemigroup of $U$, where $\pi: U * V \rightarrow V$ is the projection morphism. Conversely, let $S$ be an $E$-solid regular semigroup and let $\phi: S \rightarrow G$ be a morphism onto a group in $\mathbf{K}$ such that $1 \phi^{-1} \in \mathbf{U}$. Recall from $\S 2$ that the weak derived semigroupoid $W_{\phi}$ of $\phi$ is regular. Clearly its local semigroups are all isomorphic to $1 \phi^{-1}$. Under the hypotheses, $W_{\phi} \in \mathbf{l} \mathbf{U}$ and $\mathbf{l} \mathbf{U}=\mathbf{g} \mathbf{U}$. Applying Result $2.2, W_{\phi}$ regularly divides a semigroup $V$ in $\mathbf{U}$, and by Result 2.1 there is a regular division $\theta: W_{\phi} \rightarrow V$. Define a relation $\psi$ from $S$ to $V$ wr $G$ by

$$
s \psi=\{(\alpha, s \phi) \in V \operatorname{wr} G: g \alpha \in(g, s) \theta \forall g \in G\} .
$$

That $\psi$ is a relational morphism is routinely checked (cf. [37, p.116]). Let $s_{1}, s_{2} \in S$ and suppose $(\alpha, h) \in s_{1} \psi \cap s_{2} \psi$. Then $s_{1} \phi=h=s_{2} \phi$, so that $\left(1, s_{1}\right)$ and $\left(1, s_{2}\right)$ are coterminal in $W_{\phi}$, and $1 \alpha \in\left(1, s_{1}\right) \theta \cap\left(1, s_{2}\right) \theta$. Since $\theta$ is a division, $\left(1, s_{1}\right)=\left(1, s_{2}\right)$ and $s_{1}=s_{2}$. Hence $\psi$ is a division.

Recall that $\# \theta$ is a regular subsemigroupoid of $W_{\phi} \times V$ that projects onto $W_{\phi}$. To prove the proposition we show that $\operatorname{Reg} \# \psi$ is a (regular) subsemigroup of \# $\psi$ that projects onto $S$. The associated relational morphism is then a regular division $S \rightarrow V \operatorname{wr} G \in \mathbf{U} * \mathbf{K}$.

To show the regular elements form a subsemigroup it suffices, by Result 1.1, to show that the product of any two idempotents of $\# \psi$ is again regular. Since $\# \psi \leq S \times(V \operatorname{wr} G)$, its idempotents have the form $(e,(\alpha, 1))$, where $e \in E_{S}$ and $\alpha \in E_{V^{G}}$. Let $(e,(\alpha, 1)),(f,(\beta, 1))$ be two such idempotents. Their product is $(e f,(\alpha \beta, 1))$. Now $V$, and therefore $V^{G}$, is $E$-solid and $\alpha \beta$ has $\mathcal{H}$-related inverse $(\alpha \beta)^{-1}$; for each $g \in G, g(\alpha \beta)^{-1}$ is the $\mathcal{H}$-related inverse of $g(\alpha \beta)$ in $V$. Similarly, since $S$ is $E$-solid, ef has $\mathcal{H}$-related inverse $(e f)^{-1}$. But in $W_{\phi}$, for any $g \in$ $G,(g, e)$ and $(g, f)$ are loops at $g$, so that $\left(g,(e f)^{-1}\right)$ is the $\mathcal{H}$-related inverse of $(g, e f)$ in $\left(W_{\phi}\right)_{g}$. By hypothesis, $((g, e), g \alpha)$ and $((g, f), g \beta)$ belong to $\# \theta$, in 
fact to the local semigroup \# $\# \theta_{(g, 1)}$, which is $E$-solid, since $\left(W_{\phi}\right)_{g}$ and $V$ are $E$ solid. Hence $\left(\left(g,(e f)^{-1}\right), g(\alpha \beta)^{-1}\right)=((g, e f), g \alpha \beta)^{-1} \in \# \theta$, for all $g \in G$. Thus $\left((e f)^{-1},\left((\alpha \beta)^{-1}, 1\right)\right) \in \# \psi$, and clearly this element is an inverse for $(e f,(\alpha \beta, 1))$. Hence $\operatorname{Reg} \# \psi \leq \# \psi$.

Next let $s \in S$. We must find $\alpha \in V^{G}$ such that $(s,(\alpha, s \phi)) \in \operatorname{Reg} \# \psi$. Fix $s^{\prime} \in V(s)$ and let $g \in G$. Since $G$ is a group, $\left(g s \phi, s^{\prime}\right) \in V((g, s))$; and since \# $\theta$ is regular and the projection on $W_{\phi}$ is quotient, there exist mutually inverse elements $v_{g}, v_{g}^{\prime}$ of $V$ such that $\left((g, s), v_{g}\right)$ and $\left(\left(g s \phi, s^{\prime}\right), v_{g}^{\prime}\right)$ belong to $\# \theta$. Define $\alpha, \beta: G \rightarrow V$ by $g \alpha=v_{g}, g \beta=v_{g s^{\prime} \phi}^{\prime}, g \in G$. Then for all $g \in G, g \alpha \in(g, s) \theta$ and $(g s \phi) \beta \in\left(g s \phi, s^{\prime}\right) \theta$; that is, since $G$ is a group, $g \beta \in\left(g, s^{\prime}\right) \theta$ for all $g \in G$. By definition, $(s,(\alpha, s \phi)),\left(s^{\prime},\left(\beta, s^{\prime} \phi\right)\right) \in \# \psi$, and it may be routinely verified that these elements are mutually inverse.

The final sentence is immediate from the results of $\S 2$.

The equalities $\mathbf{A} * \mathbf{K}=\mathbf{A m K}$, for band varieties $\mathbf{A}$ (and group varieties $\mathbf{K}$ ), were essentially proved in [19].

Finally, the main result of this section is now immediate from the proposition and Corollary 1.9.

Theorem 5.2. For any e-local e-variety $\mathbf{U}$ of E-solid semigroups, $C_{\infty} \mathbf{U}=\mathbf{U} * \mathbf{G}$. Therefore the following equations hold:

(i) $C_{\infty} \mathbf{C R}(\mathbf{H})=\mathbf{C R}(\mathbf{H}) * \mathbf{G}$, for all group varieties $\mathbf{H}$;

(ii) in particular, $\mathbf{E S}=\mathbf{C R} * \mathbf{G}$ and $\mathbf{O}=\mathbf{B} * \mathbf{G}$;

(iii) $C_{\infty} \mathbf{A}=C \mathbf{A}=\mathbf{A} * \mathbf{G}$ for every band variety $\mathbf{A}$;

(iv) in particular, $\mathbf{I}=\mathbf{S} * \mathbf{G}$ and $\mathbf{G I}=\mathbf{N B} * \mathbf{G}$.

5.2. Decompositions of the form $\mathbf{G} *_{-}$. In this subsection we describe the evarieties of the form $\mathbf{G} * \mathbf{V}$, where $\mathbf{V}$ is a nontrivial variety of completely regular semigroups, as Mal'cev products $\mathbf{L G m V}$. The Mal'cev product possesses a major advantage over the semidirect product in this context [12]: $\mathbf{L} \mathbf{G m} \mathbf{V}$ consists precisely of those regular semigroups $S$ that possess a congruence $\rho$ such that (a) $S / \rho \in \mathbf{V}$ and (b) each idempotent $\rho$-class belongs to LG. (That is, this class is already closed under quotients). Such Mal'cev products have played an important role in the study of completely regular semigroup varieties, in terms of the operator $(-)^{T_{l}}$ [29]. (For more on this operator see $\S 8.2$.)

Our result generalizes its 'well-known' analogue for pseudovarieties (whose proof remains valid for varieties of bounded exponent) [16, Proposition 4.2]. It is shown in [16, Proposition 4.2] that the semidirect product of a group with a completely regular semigroup is again completely regular. The techniques used herein are similar to those of the preceding subsection, but this time using the derived semigroupoid rather than its weak version. In the context of this section, however, these semigroupoids need not be regular. Whether our results may be extended to cover all cases where the first factor is completely simple and the second factor is $E$-solid we do not know at this point.

A groupoid is a category in which every edge $e$ is invertible; we denote the (necessarily unique) inverse of $e$ by $e^{-1}$. The connected components of a groupoid are then strongly connected; it is well known (cf. [37, Proposition 3.7]) that any connected groupoid $C$ is 'equivalent' to a group. In particular, there is a faithful category morphism from $C$ to any one of its local groups, respecting the inverse operation. 
A category all of whose local monoids are groups need not be a groupoid. However, its strongly connected components are groupoids [37, Proposition 7.5]. Clearly, the converse also holds. Observe, at this point, that for any successive edges $e, f$ of a strongly connected component of such a category, $f^{-1} e^{-1}=(e f)^{-1}$. That is, each subgroupoid is involutory, in the sense of [33]. Now Tilson [37] showed that every category that is locally a group divides some group. However, we need a stronger property, that there exists a division that respects the inverses of invertible elements.

Proposition 5.3. Let $C$ be a category all of whose strongly connected components are groupoids. Then there is a division $\theta: C \rightarrow H$, for some group $H$, such that if $(e, h) \in \theta$ for some invertible edge $e$ and some $h \in H$, then $\left(e^{-1}, h^{-1}\right) \in \theta$.

Proof. Let $G$ be any graph and let $G \bullet$ be the free category on the graph obtained by adjoining, for each edge $x: c \rightarrow d$ in the union of its strongly connected components, a new edge $x^{\prime}: d \rightarrow c$. Put $x^{\prime \prime}=x$ for each such $x$. For any path $p=p_{1} p_{2} \cdots p_{n}$ in a strongly connected component, put $p^{\prime}=p_{n}^{\prime} \cdots p_{2}^{\prime} p_{1}^{\prime}$ and $p^{\prime \prime}=p$. Then $G^{\bullet}$ is free with respect to the property that each such component is an involutory category. Let $\kappa$ be the congruence on $G^{\bullet}$ generated by all the pairs $\left(x x^{\prime}, 1_{t(x)}\right)$, for $x$ or $x^{\prime}$ an edge in a strongly connected component of $G$. Clearly, $p p^{\prime} \kappa 1_{t(p)}$ for all paths in such a component. Thus the strongly connected components of $G^{\bullet} / \kappa$ are all groupoids. The reduced form of a path in $G^{\bullet}$ is the path $\bar{p}$ obtained by deleting any subpath $u u^{\prime}$, where $u$ lies in a strongly connected component of $G$. Clearly $p \kappa \bar{p}$.

It follows from the paragraph preceding the statement of the proposition that there is a graph $G$ and a quotient morphism $\psi: G^{\bullet} / \kappa \rightarrow C$ such that $\left(p^{\prime} \kappa\right) \psi=$ $(p \kappa \psi)^{-1}$ for all paths $p$ in strongly connected components of $G$.

Similarly to $\S 2$, there is a faithful morphism $\eta: G^{\bullet} \rightarrow(E G)^{\bullet}$ (the free involutory monoid on $E G$ ) obtained by regarding a path in $G^{\bullet}$ as an involutory word over the set $E G$. Let $\rho_{\mathbf{G}}$ denote the least group congruence on $(E G)^{\bullet}$. Let $p$ be a path in $G^{\bullet}$. Then, using the usual definition of reduced form in $(E G)^{\bullet}$, we have $\overline{p \eta}=\bar{p} \eta$. Thus $\kappa$ is just the congruence on $G^{\bullet}$ induced from $\rho_{\mathbf{G}}$ under $\eta$. There is an induced faithful morphism $\bar{\eta}: G \bullet / \kappa \rightarrow(E G)^{\bullet} / \rho_{\mathbf{G}}$, and for all paths $p$ in some strongly connected component of $G, p^{\prime} \kappa \bar{\eta}=(p \kappa \bar{\eta})^{-1}$. Hence the composition $\psi^{-1} \bar{\eta}$ has the requisite properties.

\section{Lemma 5.4. CSmCR $\subseteq \mathrm{CR}$.}

Proof. We use a result of Hall [8, Theorem 4.1.2]: a regular semigroup is completely regular if and only if $x^{2}\left(x^{3}\right)^{\prime} x^{2}=x$ for every $x$ and for every inverse $\left(x^{3}\right)^{\prime}$ of $x^{3}$. Now suppose $S$ is regular, $T$ is completely regular and $\phi$ is a morphism of $S$ upon $T$ such that $e \phi^{-1} \in \mathbf{C S}$ for all $e \in E_{T}$. Let $x \in S$, let $x^{\prime} \in V(x)$ and let $\left(x^{3}\right)^{\prime}$ be any inverse of $x^{3}$. Then $\left(x^{3}\right)^{\prime} \phi \in V\left((x \phi)^{3}\right)$, so, since $T$ is completely regular, $\left(x^{2}\left(x^{3}\right)^{\prime} x^{2}\right) \phi=x \phi$. Hence $\left(x^{2}\left(x^{3}\right)^{\prime} x^{2} x^{\prime}\right) \phi=\left(x x^{\prime}\right) \phi$. By hypothesis, $\left(x x^{\prime}\right) \phi \phi^{-1}$ is completely simple, so $x^{2}\left(x^{3}\right)^{\prime} x^{2} x^{\prime}$ and $x x^{\prime}$ are $\mathcal{H}$-related idempotents, hence equal. Thus $x^{2}\left(x^{3}\right)^{\prime} x^{2}=x$, and, by Hall's result, $S$ is completely regular.

Theorem 5.5. Let $\mathbf{V}$ be a variety of completely regular semigroups that does not consist entirely of groups. Then $\mathbf{G} * \mathbf{V}=\mathbf{L} \mathbf{G m V}=\mathbf{V}^{T_{l}}$.

Proof. Consider first a semidirect product $U * V$, where $U \in \mathbf{G}, V \in \mathbf{V}$. Let $e \in E_{V}$ and suppose $(a, e)$ is an idempotent in $e \pi^{-1}$, where $\pi$ is the projection of $U * V$ onto its second component. Then ${ }^{e} a=1$, and so, for any $b \in U,(b, e)(a, e)=(b, e)$. 
Any $(g, e) \in e \pi^{-1}$ has an inverse in $e \pi^{-1}$, for instance $\left({ }^{e}\left(g^{-1}\right), e\right)$. Hence $e \pi^{-1}$ is a left group. Thus $U * V \in \mathbf{L G m V}$.

Conversely, suppose $S$ is a regular semigroup and $\phi$ is a morphism of $S$ upon a semigroup $T \in \mathbf{V}$, with the property that $e \phi^{-1} \in \mathbf{L} \mathbf{G}$ for all $e \in E_{T}$. By Lemma 5.4, $\mathrm{S}$ is also completely regular. It suffices to consider the situation where $S$ is free in $\mathbf{L G m V}$. If $S$ is free of rank one, then it is a group and thus belongs to $\mathbf{G} * \mathbf{V}$. Otherwise, since $\mathbf{L} \mathbf{G m} \mathbf{V}$ contains $\mathbf{L G}, S$ is not a monoid, whence neither is $T$, since the congruence induced by $\phi$ is contained in $\mathcal{L}$.

Consider the derived semigroupoid $D_{\phi}$ (see $\left.\S 2\right)$. Thus $V D_{\phi}=T^{1}$ and $E D_{\phi}=$ $\left(T^{1} \times S\right) / \equiv$, where $[t, s]: t \rightarrow t(s \phi)$. Two coterminal pairs $\left(t, s_{1}\right),\left(t, s_{2}\right)$ are $\equiv-$ related if $u s_{1}=u s_{2}$ for all $u \in t \phi^{-1}$. The local subsemigroup $\left(D_{\phi}\right)_{1}=\{[1,1]\}$. In addition, $[1,1][1, s]=[1, s]$ for all $s \in S$. Let $t \in T$ and let $u \in t \phi^{-1}$, with inverse $u^{\prime}$. There is an idempotent loop $[t, e]$ at $t$, for some $e \in E_{S}$ (for instance $u^{\prime} u$ ). Then $\left(u^{\prime} u e\right) \phi=\left(u^{\prime} \phi\right) t(e \phi)=\left(u^{\prime} \phi\right) t=\left(u^{\prime} u\right) \phi$. By hypothesis, $u^{\prime} u e \mathcal{L} u^{\prime} u$, whence $u e \mathcal{L} u$ and so $u e=u$. It follows that $\left(D_{\phi}\right)_{t}$ contains exactly one idempotent. Moreover, in the same context, for any edge $[t, s],[t, e][t, s]=[t, e s]=[t, s]$, since ues $=u s$; and for any edge $\left[t_{1}, r\right]$ such that $t_{1}(r \phi)=t$ and any $v \in t_{1} \phi^{-1}, v r \in t \phi^{-1}$, so that vre $=v r$ and $\left[t_{1}, r\right][t, e]=\left[t_{1}, r\right]$. Hence $D_{\phi}$ is actually a category. Observe that if $t \in T, s \in S, s^{\prime} \in V(s)$ and $t\left(s s^{\prime}\right) \phi=t$, then $[t, s]$ is invertible and $[t, s]^{-1}=$ $\left[t(s \phi), s^{\prime}\right]$. Thus all the local monoids of $D_{\phi}$ are groups.

By Proposition 5.3, there is a division $\theta: D_{\phi} \rightarrow H$, for some group $H$, with the property that if $[t, s]$ is invertible and $([t, s], h) \in \theta$ then $\left([t, s]^{-1}, h^{-1}\right) \in \theta$. Similarly to the proof of Proposition 5.1 (and [37, Theorem 5.2]), define a relation $\psi$ from $S$ to $H$ wr $T$ by

$$
s \psi=\{(\alpha, s \phi) \in H \mathrm{wr} T: t \alpha \in[t, s] \theta \forall t \in T\} .
$$

That $\psi$ is a relational morphism is again routinely checked. The argument that it is a division is similar to that of the cited proposition, making use now of the fact that if $\left[1, s_{1}\right]=\left[1, s_{2}\right]$, then since $1 \in 1 \phi^{-1}, s_{1}=s_{2}$.

We show $\psi$ is regular. Let $(s,(\alpha, s \phi)) \in \psi$. As noted above, both $S$ and $H$ wr $T$ are completely regular. Recall that for an element $a$ of a completely regular semigroup, $a^{-1}$ and $a^{0}$ denote the inverse of $a$ and the identity element, respectively, in the subgroup $H_{a}$. Thus $a^{0}=a a^{-1}=a^{-1} a$. We show that $\left(s^{-1},(\alpha, s \phi)^{-1}\right) \in \psi$. It can be easily verified (or see [16, Proposition 4.1]) that $(\alpha, s \phi)^{-1}=\left(\alpha\left({ }^{(s \phi)^{0}} \alpha\right)^{-1}\left({ }^{(s \phi)^{-1}} \alpha\right)^{-1},(s \phi)^{-1}\right)$. Now for any $t \in T$,

$$
t\left(\alpha\left({ }^{(s \phi)^{0}} \alpha\right)^{-1}\left({ }^{(s \phi)^{-1}} \alpha\right)^{-1}\right)=(t \alpha)\left(\left(t(s \phi)^{0}\right) \alpha\right)^{-1}\left(\left(t(s \phi)^{-1}\right) \alpha\right)^{-1} .
$$

By assumption, $t \alpha \in[t, s] \theta$. Similarly, $\left(t(s \phi)^{0}\right) \alpha \in\left[t(s \phi)^{0}, s\right] \theta$. But $\left[t(s \phi)^{0}, s\right]$ is invertible, with inverse $\left[t(s \phi), s^{-1}\right]$, so by the assumption on $\theta,\left(\left(t(s \phi)^{0}\right) \alpha\right)^{-1} \in$ $\left[t(s \phi), s^{-1}\right] \theta$. Similarly, $\left(\left(t(s \phi)^{-1}\right) \alpha\right)^{-1} \in\left[t(s \phi)^{0}, s^{-1}\right] \theta$. Hence

$$
\begin{aligned}
& t\left(\alpha\left({ }^{(s \phi)^{0}} \alpha\right)^{-1}\left({ }^{(s \phi)^{-1}} \alpha\right)^{-1}\right) \in[t, s] \theta\left[t(s \phi), s^{-1}\right] \theta\left[t(s \phi)^{0}, s^{-1}\right] \theta \\
& \subseteq\left[t, s s^{-1} s^{-1}\right] \theta=\left[t, s^{-1}\right] \theta \text {, }
\end{aligned}
$$

as required.

Since $\mathbf{L} \mathbf{G m} \mathbf{G}=\mathbf{L} \mathbf{G}$ but $\mathbf{G} * \mathbf{G}=\mathbf{G}$, that $\mathbf{V}$ be a nongroup variety is essential. 


\section{FreE SEMIGROUPS IN PRODUCTS OF SEMIGROUP VARIETIES}

In this section we outline the standard description of free semigroups in semidirect products of semigroup varieties (in the usual universal algebraic sense), together with a graphical version, which provides more information, to give the reader an aid to understanding of the 'regular' analogues that follow in the sequel.

The former description has a history dating back to B.H. Neumann (cf. [28]) for products of group varieties. We outline a proof of its validity, somewhat different from that presented by J. Almeida [1], that is transparent and ideally suited to modification to other situations. In essence, for any varieties $\mathbf{U}$ and $\mathbf{V}$ and any nonempty set $X$ we construct a semidirect product $S * T$ in $\mathbf{U} * \mathbf{V}$ containing a copy of $X$, with the property that any map of $X$ into a semidirect product $U * V$, where $U \in \mathbf{U}$ and $V \in \mathbf{V}$, extends to a morphism $S * T \rightarrow U * V$. The subsemigroup of $S * T$ generated by the copy of $X$ is $F(\mathbf{U} * \mathbf{V})_{X}$. Describing $F(\mathbf{U} * \mathbf{V})_{X}$ in this way is often referred to as "cutting (the semidirect product) down to generators".

The graphical version was outlined to us by S.W. Margolis, for the categorical situation, but has not previously appeared in print, to the authors' knowledge. We shall derive it from the semigroup-theoretic version. (A direct proof is possible by means of the 'Derived Category Theorem' of Tilson [37].) Whilst perhaps forbidding to the uninitiated, it is simply stated once the language has become familiar. Its advantage is that, in comparison with the former description, which 'solves the word problem' in the free semigroup in a semidirect product (relative to the free semigroups in the original varieties), the graphical version also produces a set of canonical forms. The added power of this version will become especially apparent in the e-varietal context.

6.1. The semigroup-theoretic approach. Let $\mathbf{U}, \mathbf{V}$ be varieties of semigroups and let $X$ be a nonempty set. The free semigroup on $X$ in $\mathbf{V}$ is denoted $F \mathbf{V}_{X}$, and similarly for $\mathbf{U}$. Following the principle espoused earlier, the semidirect product $\mathbf{U} * \mathbf{V}$ is the variety generated by all semidirect products $S * T, S \in \mathbf{U}, T \in \mathbf{V}$, for which the action of $T$ on $S$ is left unitary whenever $T$ is a monoid. Thus $\mathbf{U} * \mathbf{V}$ comprises all semigroups that divide such a semidirect product, in the usual sense of division [37].

For convenience, denote $F \mathbf{V}_{X}$ by $T$ and $F \mathbf{U}_{T^{1} \times X}$ by $S$. There is a natural action of $T$ on $S$, as follows. The semigroup $T$ acts on the set $T^{1} \times X$ by ${ }^{t}\left(t_{1}, x\right)=\left(t t_{1}, x\right)$. For each $t \in T^{1}$, denote by $\lambda_{t}$ the induced transformation of $T^{1} \times X ; \lambda_{t}$ extends uniquely to an endomorphism $\bar{\lambda}_{t}$, say, of $S$, which we again denote by $s \rightarrow{ }^{t} s$. This yields an appropriate action of $T$ on $S$ and thus a well defined semidirect product $S * T$.

Define $i: X \rightarrow S * T$ by $x i=((1, x), x), x \in X$.

Result 6.1. Let $\mathbf{U}, \mathbf{V}$ be varieties of semigroups and let $X$ be a nonempty set. Then $F(\mathbf{U} * \mathbf{V})_{X}$ is isomorphic to the subsemigroup of $F \mathbf{U}_{F \mathbf{V}_{X}^{1} \times X} * F \mathbf{V}_{X}$ generated by $\{((1, x), x): x \in X\}$.

Proof. Let $U \in \mathbf{U}, V \in \mathbf{V}$, with $V$ acting on $U$, left unitarily if $V$ is a monoid. Again, let $\lambda_{v}$ denote the endomorphism of $U$ associated with $v \in V$. Suppose $\phi: X \rightarrow U * V$ is an arbitrary map. For each $x \in X$ put $x \phi=\left(x_{U}, x_{V}\right)$. Define $\beta: T \rightarrow V$ to be the unique extension to $T$ of the map $x \rightarrow x_{V}$. Also denote by $\beta$ its extension to a morphism $T^{1} \rightarrow V^{1}$, where $1 \beta=1$. (Note that if $T$ is already a monoid then $\mathbf{V}$ consists entirely of groups, and so there is no conflict 
with the original value of $1 \beta$.) Next define $\alpha: T^{1} \times X \rightarrow U$ by $(t, x) \alpha={ }^{t \beta} x_{U}$ and extend it to a morphism $\alpha: S \rightarrow U$. It is easily verified that the composite morphisms $\bar{\lambda}_{t} \alpha, \alpha \lambda_{t \beta}$ agree on $T^{1} \times X$, and so agree on $S$. Thus $\left({ }^{t} s\right) \alpha={ }^{t \beta} s \alpha$, for all $t \in T, s \in S$. Hence the product map $\theta=\alpha \times \beta: S * T \rightarrow U * V$ is a morphism. Moreover, for any $x \in X, x i \theta=x \phi$.

Thus for any function $\phi: X \rightarrow U * V$ there is a morphism $\theta: S * T \rightarrow U * V$ such that $i \theta=\phi$. With the sequel in mind, it is convenient to separate the last part of the proof as a lemma. Its proof contains some subtleties that, while transparent in this situation, become more consequential in the e-varietal context.

Lemma 6.2. Let $\mathbf{C}$ be a class of semigroups that is closed under products and let $\mathbf{V}$ be the variety that it generates. Let $X$ be a nonempty set. A semigroup $W \in \mathbf{V}$, together with a map $i: X \rightarrow W$, is free in $\mathbf{V}$ on $X$ if and only if $W=\langle X i\rangle$ and for all $C \in \mathbf{C}$ and for all maps $\phi: X \rightarrow C$, there is a morphism $\bar{\phi}: W \rightarrow C$ such that $i \bar{\phi}=\phi$.

Proof. Necessity is clear. Conversely, let $A \in \mathbf{V}$ and $\theta: X \rightarrow A$. By standard universal algebraic arguments there exist $C \in \mathbf{C}$, a subsemigroup $B$ of $C$ and a morphism $\pi$ of $B$ onto $A$. For each $x \in X$, choose $b_{x} \in B$ such that $b_{x} \pi=x \theta$ and define $\phi: X \rightarrow B \subseteq C$ by $x \phi=b_{x}$. By hypothesis there exists a morphism $\bar{\phi}: W \rightarrow C$ such that $i \bar{\phi}=\phi$. Since $B$ contains $X \phi=X i \bar{\phi}$, it contains $\langle X i\rangle \bar{\phi}=W \bar{\phi}$. Hence the composite $\bar{\theta}=\bar{\phi} \pi$ is a morphism satisfying $i \bar{\theta}=\theta$. Since $X i$ generates $W, \bar{\theta}$ uniquely possesses this property, and so $S$, being a member of $\mathbf{V}$, is free.

Since $\mathbf{U} * \mathbf{V}$ is generated by $\{U * V: U \in \mathbf{U}, V \in \mathbf{V}\}$, the lemma completes the proof of the result.

6.2. The graphical approach. We continue the notation of the previous subsection. Let $G$ be the graph with $V G=T^{1}$ and $E G=T^{1} \times X$, where $(t, x)$ : $t \rightarrow t x$. Let $G^{+}$denote the free semigroupoid on $G$ (see $\S 2$ ). Thus its vertex set is again $T^{1}$ and its edges are the (nonempty) paths in $G$, which are of the form $p=\left(t, x_{1}\right)\left(t x_{1}, x_{2}\right) \cdots\left(t x_{1} \cdots x_{n-1}, x_{n}\right)$, where $t \in T^{1}$ and each $x_{i}$ belongs to $X$. It is worthwhile remarking that $G^{+}$is isomorphic to the weak derived semigroupoid (see $\S 2$ ) of the morphism $X^{+} \rightarrow T$, under the edge-correspondence $p \rightarrow\left(t, x_{1} \cdots x_{n}\right)$, the vertex sets being identical.

Let $\eta: G^{+} \rightarrow(E G)^{+}$be the morphism defined in $\S 2: \eta$ collapses all vertices and maps each path in $G$ to the corresponding word over the set of edges. Denote by $\rho_{\mathbf{U}}$ the least congruence on $(E G)^{+}$for which the quotient belongs to $\mathbf{U}$. Thus $\rho_{\mathbf{U}}$ is fully invariant and $(E G)^{+} / \rho_{\mathbf{U}} \cong F \mathbf{U}_{T^{1} \times X}$, which is the semigroup we have been denoting by $S$. As usual, we regard $E G$ as a subset of $S$.

Let $\rho$ be the congruence that $\rho_{\mathbf{U}}$ induces on $G^{+}$via $\eta$; that is, paths $p$ and $q$ in $G$ are $\rho$-related if and only if they are coterminal and $p \eta$ and $q \eta$ are $\rho_{\mathbf{U}}$-related as words of $(E G)^{+}$. Then $G^{+} / \rho$ is a semigroupoid with vertex set $T^{1}$ and edge set $E G^{+} / \rho$; there is a quotient morphism, which we again denote $\rho$, from $G^{+}$to $G^{+} / \rho$; and there is an induced faithful morphism $\bar{\eta}: G^{+} / \rho \rightarrow(E G)^{+} / \rho_{\mathbf{U}}=S$. (Similarly to [15, Proposition 2.1], it can be shown that $G^{+} / \rho$ is isomorphic to the free semigroupoid on $G$ in the variety $\mathbf{g} \mathbf{U}$; starting from that point, a proof of the theorem below could be made independently of $\S 6.1$.)

Now the semigroup $T$ acts on $T^{1}=V G$ by left translation, and on $T^{1} \times X$, as before: ${ }^{t_{1}}(t, x)=\left(t_{1} t, x\right)$. The latter action extends to an action of $T$ on 
$\left(T^{1} \times X\right)^{+}=(E G)^{+}$. Since $\rho_{\mathbf{U}}$ is fully invariant, whenever $u \rho_{\mathbf{U}} v$ in $(E G)^{+}$and $t_{1} \in T$, we have ${ }^{t_{1}} u \rho_{\mathbf{U}}{ }^{t_{1}} v$.

Moreover, since the action of $T$ on $E G$ respects heads and tails of edges, then in combination with the action on $V G$ there is an action of $T$ on the semigroupoid $G^{+}$. (See $\$ 7.2$ for more on actions of semigroups on semigroupoids.) Since the action is induced via $\eta$ from that on $(E G)^{+}$, it is compatible with the congruence $\rho$. Hence there is an induced action of $T$ on $G^{+} / \rho$.

We now associate semigroups with $G^{+}$and $G^{+} / \rho$. That associated with the latter will turn out to be free on $X$ in $\mathbf{U} * \mathbf{V}$. Let $S\left(G^{+}\right)=\bigcup_{t \in T} G^{+}(1, t)$, with product $p \star q=p^{h(p)} q$; and let $S\left(G^{+} / \rho\right)=S\left(G^{+}\right) / \rho$ (the image of $S\left(G^{+}\right)$under the edge component of the quotient morphism $\rho$ ). In view of the previous paragraph, $S\left(G^{+} / \rho\right)$ may be defined in the same way that $S\left(G^{+}\right)$was, that is, $S\left(G^{+} / \rho\right)=$ $\bigcup_{t \in T}\left(G^{+} / \rho\right)(1, t)$, with product $p \rho \star q \rho=p \rho^{h(p)} q \rho$.

Define a map $j: X \rightarrow S\left(G^{+} / \rho\right)$ by $x j=(1, x) \rho$.

Theorem 6.3. Let $\mathbf{U}$ and $\mathbf{V}$ be varieties of semigroups and let $X$ be a nonempty set. Put $T=F \mathbf{V}_{X}$, and let $G$ be the graph with vertex set $T^{1}$ and edge set $T^{1} \times X$. Let $G^{+}$be the free semigroupoid on $G$. Let $\rho$ be the congruence on $G^{+}$induced by the least $\mathbf{U}$-congruence on the free semigroup on $E G$.

Then $F(\mathbf{U} * \mathbf{V})_{X}$ is isomorphic to the semigroup $S\left(G^{+} / \rho\right)=\left(\bigcup_{t \in T} G^{+}(1, t)\right) / \rho$, with product $p \rho \star q \rho=p \rho^{h(p)} q \rho$, for paths $p$ and $q$ in $G$ beginning at 1 .

Proof. We show $S\left(G^{+} / \rho\right)$ is isomorphic to the subsemigroup $\langle X i\rangle=\langle((1, x), x)$ : $x \in X\rangle$ of $S * T$ described in Result 6.1. Define a map $\psi: S\left(G^{+} / \rho\right) \rightarrow S * T$ by $(p \rho) \psi=((p \rho) \bar{\eta}, h(p))$. It is routinely checked that $\psi$ is a morphism. Suppose $p$ and $q$ are paths in $G$ beginning at 1 such that $p \rho \psi=q \rho \psi$. Then since $h(p)=h(q), p$ and $q$ are coterminal, as are $p \rho$ and $q \rho$. But $\bar{\eta}$ is faithful, so $p \rho=q \rho$. Hence $\psi$ is injective.

For $x \in X, x j \psi=(1, x) \rho \psi=((1, x) \rho \bar{\eta}, x)=\left((1, x) \eta \rho_{\mathbf{U}}, x\right)=((1, x), x)$. So by the cited result, it suffices to show that $S\left(G^{+} / \rho\right)=\langle X j\rangle$. But for any path $p \in$ $S\left(G^{+}\right), p=\left(1, x_{1}\right)\left(x_{1}, x_{2}\right) \cdots\left(x_{1} \cdots x_{n-1}, x_{n}\right)$, say, $p=\left(1, x_{1}\right) \star\left(1, x_{2}\right) \star \cdots \star\left(1, x_{n}\right)$. So $S\left(G^{+}\right)=\langle(1, x): x \in X\rangle$, and the morphic property of $\rho$ yields the desired equality.

\section{E-FREE $E$-SOLID SEMIGROUPS}

More generally than the heading suggests, we consider the e-free semigroups in semidirect products $\mathbf{U} * \mathbf{V}$, where $\mathbf{U} \subseteq \mathbf{E S}$ and $\mathbf{V} \subseteq \mathbf{G}$. By Proposition 3.11 such products lie within $\mathbf{E S}$, and so all their e-free semigroups exist. Keeping in mind Theorem 5.2, we shall be particularly interested in the cases where $\mathbf{U}$ is completely regular. Refer to $\S 5.1$ for various alternative descriptions, such as Mal'cev products, of the e-varieties under consideration.

Following the general scheme outlined in the preceding section, we proceed in two steps, a purely 'regular semigroup-theoretic' step and a 'graph-theoretic' refinement. Roughly speaking, the former solves a version of the 'word problem' and the latter determines a set of canonical forms for the elements of the e-free semigroups.

7.1. The semigroup-theoretic approach. Let $\mathbf{U}$ be an e-variety of $E$-solid semigroups and let $\mathbf{V}$ be a variety of groups, and let $X$ be a nonempty set. Let $X^{\prime}$ and $\bar{X}$ be as in $\S 1$. For convenience, denote $F \mathbf{V}_{X}\left(=E F \mathbf{V}_{X}\right)$ by $T\left(=T^{1}\right)$ and denote $E F \mathbf{U}_{T \times X}$ by $S$. We may regard $T$ as being generated, as a group, by the set $X$ 
itself. In that case, $x^{\prime}$ is identified with $x^{-1}$. Similarly, we may regard $S$ as being generated as a regular semigroup by $\overline{T \times X}=(T \times X) \cup(T \times X)^{\prime}$. However, a nice technical device (introduced by Szendrei [35] and transparent, in terms of the Cayley graph of $T$ ) is to identify this set with the set $T \times \bar{X}$, by identifying $(t, x)^{\prime}$ with $\left(t x, x^{\prime}\right)$, for all $t \in T, x \in X$. Thus we shall regard $T \times \bar{X}$ as a subset of $S$, with $\left(t x, x^{\prime}\right) \in V((t, x))$ for all $t \in T, x \in X$. In fact, in view of the equation $x^{\prime \prime}=x$ and the identification in $T$ of $x^{\prime}$ with $x^{-1}$, we may identify $(t, y)^{\prime}$ with $\left(t y, y^{\prime}\right)$ for all $t \in T, y \in \bar{X}$.

Now $T$ acts on $T \times \bar{X}$ by ${ }^{t_{1}}(t, y)=\left(t_{1} t, y\right), t \in T, y \in \bar{X}$. For each $t_{1} \in T$, denote by $\lambda_{t_{1}}$ the induced transformation of $T \times \bar{X}$. It is clear that, in terms of the above identification, $\lambda_{t_{1}}$ is a matched mapping of $T \times \bar{X}$ into $S$ and therefore extends uniquely to an endomorphism $\bar{\lambda}_{t_{1}}$ of $S$, which we again denote by $s \rightarrow{ }^{t_{1}} s$. For any $t_{1}, t_{2} \in T,(t, y) \bar{\lambda}_{t_{2}} \bar{\lambda}_{t_{1}}=(t, y) \bar{\lambda}_{t_{1} t_{2}}$, so by uniqueness of extensions, ${ }^{t_{1} t_{2}} s={ }^{t_{1}}\left({ }^{t_{2}} s\right)$ for all $s \in S$. Similarly, ${ }^{1} s=s$ for all $s \in S$. Hence there is a well defined left unitary action of $T$ on $S$, and the semidirect product $S * T$ exists and belongs to $\mathbf{U} * \mathbf{V}$.

Define $i: \bar{X} \rightarrow S * T$ by $y i=((1, y), y), y \in \bar{X}$.

Theorem 7.1. Let $\mathbf{U}$ be an e-variety of E-solid semigroups, $\mathbf{V}$ a variety of groups and $X$ a nonempty set. Let $F \mathbf{V}_{X}$ act on $E F \mathbf{U}_{F \mathbf{V}_{X} \times X}$ as above. Then $E F(\mathbf{U} * \mathbf{V})_{X}$ is isomorphic to the regular subsemigroup of $E F \mathbf{U}_{F \mathbf{V}_{X} \times X} * F \mathbf{V}_{X}$ generated by $\{((1, y), y): y \in \bar{X}\}$.

Proof. By Theorem 3.2 and Proposition 3.11, $S * T$ is an $E$-solid regular semigroup.

For all $y \in \bar{X}$,

$$
((1, y), y)\left(\left(1, y^{\prime}\right), y^{\prime}\right)((1, y), y)=\left((1, y)\left(y, y^{\prime}\right)(1, y), y\right)=((1, y), y)
$$

since $\left(y, y^{\prime}\right)=(1, y)^{\prime}$ in $S$ and $y^{\prime}=y^{-1}$ in $T$, under the identifications made earlier. A similar calculation completes the verification that $\left(\left(1, y^{\prime}\right), y^{\prime}\right) \in V(((1, y), y))$. By Result 1.6 the regular subsemigroup $\langle((1, y), y): y \in \bar{X}\rangle_{e}$ is well defined. In addition, we have proven that the map $i$ is matched.

Now let $U \in \mathbf{U}, V \in \mathbf{V}$, with the group $V$ acting left unitarily on $U$. As above, $U * V$ is an $E$-solid regular semigroup. Again, let $\lambda_{v}$ denote the endomorphism of $U$ associated with $v \in V$. Suppose $\phi: \bar{X} \rightarrow U * V$ is a matched mapping. For each $y \in \bar{X}$, put $y \phi=\left(y_{U}, y_{V}\right)$. Since $y^{\prime} \phi \in V(y \phi)$ and $V$ is a group, $y_{V}^{\prime}=y_{V}^{-1}$ and the equations $y_{U}{ }^{y_{V}} y_{U}^{\prime} y_{U}=y_{U}$ and $y_{U}^{\prime} y_{V}^{-1} y_{U} y_{U}^{\prime}=y_{U}^{\prime}$ hold. From the latter we obtain ${ }^{y_{V}} y_{U}^{\prime} y_{U}{ }^{y_{V}} y_{U}^{\prime}=y_{V} y_{U}^{\prime}$.

Define $\beta: T \rightarrow V$ to be the unique extension to $T$ of the map $x \rightarrow x_{V}, X \rightarrow V$. Clearly, $y \beta=y_{V}$, for all $y \in \bar{X}$. Next define $\alpha: T \times \bar{X} \rightarrow U$ by $(t, y) \alpha=$ ${ }^{t} y_{U}$, for all $t \in T, y \in \bar{X}$. Then $(t, y) \alpha(t, y)^{\prime} \alpha(t, y) \alpha=(t, y) \alpha\left(t y, y^{\prime}\right) \alpha(t, y) \alpha=$ ${ }^{t \beta} y_{U}{ }^{(t y) \beta} y_{U}{ }^{t \beta} y_{U}={ }^{t \beta}\left(y_{U}{ }^{y \beta} y_{U}^{\prime} y_{U}\right)={ }^{t \beta} y_{U}$, using one of the equations of the preceding paragraph. The final equation of that paragraph yields, similarly, the other equation required to verify that $(t, y)^{\prime} \alpha \in V((t, y) \alpha)$. Hence $\alpha$ is a matched mapping and so extends uniquely to a morphism, again denoted $\alpha$, from $S$ to $U$.

As in $\S 6.2$, it is easily verified that for every $t \in T$, the composite morphisms $\bar{\lambda}_{t} \alpha$ and $\alpha \lambda_{t \beta}$ agree on $T \times \bar{X}$ and so agree on $S$. Thus $\left({ }^{t} s\right) \alpha={ }^{t \beta} s \alpha$ for all $s \in S$. It follows that the product map $\theta=\alpha \times \beta: S * T \rightarrow U * V$ is a morphism. Further, for any $y \in \bar{X}$, $y i \theta=\left({ }^{1 \beta} y_{U}, y_{V}\right)=y \phi$, that is, $i \theta=\phi$.

An analogue of Lemma 6.2 is needed to complete the proof. 
Lemma 7.2. Let $\mathbf{C}$ be a class of regular semigroups that is closed under products and is contained in either $\mathbf{E S}$ or $L \mathbf{I}$, and let $\mathbf{V}$ be the e-variety it generates. Let $X$ be a nonempty set. A regular semigroup $W \in \mathbf{V}$, together with a matched mapping $i: \bar{X} \rightarrow W$, is e-free on $X$ in $\mathbf{V}$ if and only if $W=\langle\bar{X} i\rangle_{e}$ and for all $C \in \mathbf{C}$ and for all matched maps $\phi: \bar{X} \rightarrow C$ there is a morphism $\bar{\phi}: W \rightarrow C$ such that $i \bar{\phi}=\phi$.

Proof. We modify the proof of the cited lemma. Necessity is clear from the construction of e-free semigroups given by Yeh [42, Theorems 3.1.4, 3.2.4]. Conversely, let $A \in \mathbf{V}$ and let $\theta: \bar{X} \rightarrow A$ be a matched map. By Result 1.6(i), there exist $C \in \mathbf{C}$, a regular subsemigroup $B$ of $C$ and a morphism $\pi$ of $B$ on $A$. For each $x$ in $X, x \theta$ and $x^{\prime} \theta$ are mutually inverse. Thus there exist mutually inverse elements $b_{x}, b_{x}^{\prime}$, say, of $B$ such that $b_{x} \phi=x \theta$ and $b_{x}^{\prime} \phi=x^{\prime} \theta$. Define $\phi: \bar{X} \rightarrow B \subseteq C$ by $x \phi=b_{x}, x^{\prime} \phi=b_{x}^{\prime}, x \in X$. Clearly, $\phi$ is a matched map, so by hypothesis there exists a morphism $\bar{\phi}: W \rightarrow C$ such that $i \bar{\phi}=\phi$. Now by Lemma 1.7, $B$ contains $\langle\bar{X} i\rangle_{e} \bar{\phi}=W \bar{\phi}$, so the composite $\bar{\theta}=\bar{\phi} \pi$ is a well defined morphism from $W$ to $A$ that satisfies $i \bar{\theta}=\theta$. By Result 1.6(ii), $\bar{\theta}$ uniquely possesses this property. Since $W \in \mathbf{V}$, it is e-free in $\mathbf{V}$, on $X$.

Returning to the semidirect product situation, the remarks preceding Proposition 3.4 show that the lemma can be applied to the class $\mathbf{C}$ of all semidirect products $U * V, U \in \mathbf{U}, V \in \mathbf{V}$, which in conjunction with the earlier part of the proof shows that $\langle\bar{X} i\rangle_{e}$ is the requisite e-free semigroup.

If $F \mathbf{V}_{X}$ and $E F \mathbf{U}_{T \times X}$ are 'effectively described' then under the representation in the theorem two elements of $E F(\mathbf{U} * \mathbf{V})_{X}$ can be 'effectively distinguished' by examination of their components. (This informal argument may be formalized by using the syntactic approach to $E$-solid semigroups of Kadourek and M. Szendrei [20]. Since it will in a sense be encompassed by the approach in the remainder of this section, we omit the details.)

Absolutely free groups are so described over any set $X$. On the other hand (Result 1.5), the e-free semigroups in $\mathbf{C R}(\mathbf{H})$ have been described for any group variety $\mathbf{H}$, in such a way that the 'word problem' for $\operatorname{EF} \mathbf{C R}(\mathbf{H})_{X}, X$ countably infinite, is solvable provided that for $F \mathbf{H}_{X}$ is. Hence by Theorem 5.2 we obtain a concrete description of $E F C_{\infty} \mathbf{C R}(\mathbf{H})_{X}$ and, in particular, of $E F \mathbf{E} \mathbf{S}_{X}$ and $E F \mathbf{O}_{X}$.

Corollary 7.3. For any nonempty set $X, E F \mathbf{E S}_{X}$ is isomorphic to the regular subsemigroup of $E F \mathbf{C R}_{F \mathbf{G}_{X} \times X} * F \mathbf{G}_{X}$ generated by $\{((1, y), y): y \in \bar{X}\}$.

By analogy with $\S 6$, we may describe the method of representing $E F(\mathbf{U} * \mathbf{V})_{X}$ in Theorem 7.1 as "cutting (the semidirect product) down to regular generators". What the theorem does not tell us, in an effective way at least, is which ordered pairs of the semidirect product actually belong to the prescribed regular subsemigroup. To achieve this goal we take up the graphical approach promised above. First, some further background is required.

7.2. Groups acting on semigroupoids. We review briefly material from [35, $\S 2]$, which extended and modified work of Margolis and Pin [22]. We refer the reader to the former paper for details, although the flavour of our discussion is a little different. It may be instructive to compare the methods of $\S 6.2$ with those presented here.

A semigroup $T$ acts on a graph $C$ if it acts separately on $V C$ and $E C$ in such a way that for any edge $e: a \rightarrow b$ of $C$ and any $t \in T,{ }^{t} e:{ }^{t} a \rightarrow{ }^{t} b$; $T$ acts on a 
semigroupoid $C$ if it acts on the underlying graph in such a way that for consecutive edges $e$ and $f,{ }^{t}(e f)={ }^{t} e^{t} f$. The action is transitive if it is transitive on $V C$, that is, for any vertices $a, b$ of $C,{ }^{t} a=b$ for some $t \in T$. If $T$ is a monoid, then the action is left unitary if the identity element acts identically on $V C$ and on $E C$. Such an action is fixed point free if it is fixed point free on $V C$; that is, the equation ${ }^{t} a=a$ holds for some $t \in T$ and $a \in V C$ only if $t=1$.

In the remainder of the section, $T$ will be a group. Suppose $T$ acts on the semigroupoid $C$, left unitarily, transitively and fixed point freely. Distinguish a vertex $a$ of $C$. Clearly, for each $b \in V C$ there is a unique element $g_{b}$, say, of $T$ such that ${ }^{g_{b}} a=b$ (and $\left.g_{b}^{-1} b=a\right)$.

Let $S_{a}(C)=\bigcup_{c \in V C} C(a, c)$. Under the product $u \star v=u^{g_{h(u)}} v$, it is easily checked that $S_{a}(C)$ is a semigroup and the map $\sigma: S_{a}(C) \rightarrow T$ given by $u \sigma=g_{h(u)}$ is a surjective morphism. Moreover, regarding $S_{a}(C)$ as a one-vertex semigroupoid, there is a semigroupoid morphism $\pi: C \rightarrow S_{a}(C)$, collapsing all vertices and mapping the edge $u$ to ${ }^{g_{t(u)}^{-1}} u$.

A useful alternative interpretation of $S_{a}(C)$ is that it is isomorphic to the semigroup of orbits $C / T$ of $C$ under $T$. The elements of $C / T$ are the orbits [ $u$ ] of edges $u$ of $C$, under the action of $T$. Note that for any edge $u, u \pi$ is the unique edge in its orbit $[u]$ that starts at $a$. Thus there is an induced bijection $C / T \rightarrow S_{a}(C)$, $[u] \rightarrow u \pi$, and a well defined product induced on $C / T$ by $[u][v]=[u \pi \star v \pi]$, under which $C / T$ is isomorphic to $S_{a}(C)$. There is an induced morphism $\pi^{\prime}: C \rightarrow C / T$, which collapses vertices (technically, maps all vertices to the unique vertex orbit) and maps edges to their orbits; and an induced morphism $\sigma^{\prime}: C / T \rightarrow T$, given by $[u] \sigma^{\prime}=g_{t(u)}^{-1} g_{h(u)}$.

Hence $S_{a}(C) \cong S_{b}(C) \cong C / T$ for all $a, b \in V C$.

Suppose in the above situation that $\rho$ is a congruence on $C$ that is invariant with respect to the action, that is, whenever $u, v$ are $\rho$-related coterminal edges of $C$ then ${ }^{t} u$ and ${ }^{t} v$ are $\rho$-related for any $t \in T$. Then $T$ also acts left unitarily, transitively and fixed point freely on $C / \rho$, and $S_{a}(C / \rho) \cong S_{a}(C) / \rho_{a}$, where $\rho_{a}$ is the restriction of $\rho$ to $S_{a}(C)$. Alternatively, $(C / \rho) / T \cong(C / T) / \rho^{\prime}$, where $\rho^{\prime}$ is the congruence that relates $[u]$ and $[v]$ if and only if $u \pi \rho v \pi$. There is a commutative diagram involving the two morphisms $C / T \rightarrow T$ and $(C / \rho) / T \rightarrow T$ and the natural morphism associated with $\rho^{\prime}$.

Given any transitive and fixed point free action of a group $T$ on a semigroupoid $C, C$ can actually be recovered from $C / T$ and $\sigma^{\prime}$, for $C$ is isomorphic to the weak derived semigroupoid (see $\S 2$ ) of $\sigma^{\prime}$ under the vertex map $b \rightarrow g_{b}$ and the edge map $u \rightarrow\left(g_{t(u)},[u]\right)$.

(Szendrei proves considerably more. Suppose that $\phi: S \rightarrow T$ is a morphism of a semigroup $S$ onto a group $T$. Then $T$ acts on $V W_{\phi}=G$ by left translation (and thus transitively and fixed point freely) and on $E W_{\phi}=G \times S$ by ${ }^{t_{1}}(t, s)=\left(t_{1} t, s\right)$, $t_{1}, t \in T, s \in S$, and thus acts left unitarily, transitively and fixed point freely on $W_{\phi}$. Then there is an isomorphism $\theta$, say, of $S$ upon $S_{1}\left(W_{\phi}\right)$ such that $\theta \sigma=\phi$. In fact, for a fixed group $T$, there is a natural equivalence between the category of 'pointed' semigroupoids on which $T$ acts left unitarily, transitively and fixed point freely and the category of morphisms of semigroups into $T$.)

Finally, we remark that the results of this subsection remain true in the unary context, that is, for unary semigroups (especially, groups regarded as unary semigroups in the usual way), for locally unary semigroupoids (especially, locally com- 
pletely regular semigroupoids), actions by unary endomorphisms, and unary congruences on semigroups and semigroupoids. See the end of $\S 2$ for a discussion of locally unary semigroupoids.

7.3. The graphical approach. The main result of this subsection, Theorem 7.4, constructs $E F(\mathbf{U} * \mathbf{V})_{X}$ for any variety $\mathbf{U}$ of completely regular semigroups and any variety $\mathbf{V}$ of groups. The special case $\mathbf{U}=\mathbf{C R}, \mathbf{V}=\mathbf{G}$ yields the e-free $E$-solid semigroup on $X$. Other special cases were discussed in $\S 7.1$. See also the comments at the end of the current section. The method generalizes Szendrei's construction of the e-free $E$-solid semigroup [35, Theorem 4.1] and owes much to the techniques of her paper. However, a significant discrepancy arises, previously referred to at the end of $\S 2$. Her construction uses the free unary semigroupoid on the Cayley graph $G$ of the free group on $X$; ours uses that on the 'doubled' graph $\bar{G}$. Thus, when interpreted as a semidirect product (along the lines of $\S 7.1$ ), her construction lies within the product of a free completely regular semigroup with a free group, rather than within the product of an e-free completely regular semigroup (on the same set) with a free group (as in Theorem 7.1). The authors thank J. Kadourek for pointing out to us that the special properties of the absolutely free group enable our construction to be simplified, in the case of the e-free $E$-solid semigroups. Although it is possible to present the material of this subsection in the same generality as in $\S 7.1$, for the sake of simplicity we shall restrict ourselves to the situation where $\mathbf{U} \subseteq \mathbf{C R}$. We continue the notation of $\S 7.1$. We make the same identification of $\overline{T \times X}=(T \times X) \cup(T \times X)^{\prime}$ with $T \times \bar{X},(t, y)^{\prime} \leftrightarrow\left(t y, y^{\prime}\right)(t \in T, y \in \bar{X})$ that was made there. This has a natural interpretation in terms of the Cayley graph of $T$, the free group in $\mathbf{V}$ on $X$ (following [35]).

The Cayley graph $G$ of $T$ is given by $V G=T$ and $E G=T \times X$, where $(t, x)$ : $t \rightarrow t x$. In group theory, the action of the formal inverses $X^{\prime}$ is incorporated by the adjunction, implicitly or explicitly, of an edge $\left(t x, x^{\prime}\right)$ 'inverse' to $(t, x)$, for each $t \in T, x \in X$. Identifying each such natural 'inverse' edge with our formal inverse $(t, x)^{\prime}$ (see $\S 2$, following Result 2.4) allows us to identify $E G^{\prime}$ with $T \times X^{\prime}$ and $E \bar{G}=\overline{T \times X}$ with $T \times \bar{X}$ as above. Considering $E G$ simply as a set, we may also identify $(E G)^{\prime}$ with $E G^{\prime}$ and $\overline{E G}$ with $E \bar{G}$, where convenient.

Refer to the last part of $\S 2$ and the material preceding Result 1.5 for the terminology that follows. Let $G^{u}$ be the free locally unary semigroupoid on $\bar{G}$, and recall the faithful morphism $\eta: G^{u} \rightarrow(E G)^{u}$ (where the latter is essentially the free unary semigroup on $\overline{E G}$ ) which sends a unary path in $\bar{G}$ to the corresponding unary word over $\overline{E G}$.

Let $\rho_{\mathbf{U}}$ be the least $\mathbf{U}$-congruence on $(E G)^{u}$; that is, $\rho_{\mathbf{U}}$ is the congruence on $(E G)^{u}$ such that $(E G)^{u} / \rho_{\mathbf{U}}$ is isomorphic to $E F \mathbf{U}_{E G}=E F \mathbf{U}_{T \times X}$, which is the semigroup we have been denoting $S$. It is well known and easily proved that $\rho_{\mathbf{U}}$ is a unary congruence. As usual, we regard $\overline{E G}$ as a subset of $S$.

Let $\tau_{\mathbf{U}}$ be the congruence that $\rho_{\mathbf{U}}$ induces on $G^{u}$ via $\eta$; that is, unary paths $p$ and $q$ in $G$ are $\tau_{\mathbf{U}}$-related if and only if they are coterminal and $p \eta$ and $q \eta$ are $\rho_{\mathbf{U}}$-related as unary words of $(E G)^{u}$. Since $\rho_{\mathbf{U}}$ and $\eta$ are unary, so is $\tau_{\mathbf{U}}$. Then $G^{u} / \tau_{\mathbf{U}}$ is a locally unary semigroupoid with vertex set $T$ and edge set $(E G)^{u} / \tau_{\mathbf{U}}$; there is a quotient unary morphism from $G^{u}$ to $G^{u} / \tau_{\mathbf{U}}$ and an induced faithful unary morphism $\bar{\eta}: G^{u} / \tau_{\mathbf{U}} \rightarrow(E G)^{u} / \rho_{\mathbf{U}}=S$. (Similarly to the remarks at the corresponding point in $\S 6.2$, it can be shown that $G^{u} / \tau_{\mathbf{U}}$ is isomorphic to the e-free 
semigroupoid on $G$ in the variety $\mathbf{g} \mathbf{U}$; starting from that point, a proof of the theorem below could be made independently of $\$ 7.1$. However, a considerable amount of extra work is involved, peripheral to the task at hand, so we have preferred to proceed by applying Theorem 7.1.)

As in $\S 7.1, T$ acts on $T \times \bar{X}=\overline{E G}$ by ${ }^{t_{1}}(t, y)=\left(t_{1} t, y\right), t_{1}, t \in T, y \in \bar{X}$. The action extends to an action by unary endomorphisms of $T$ on $(E G)^{u}$, the free unary semigroup on $\overline{E G}$. Consider the definition of $\rho_{\mathbf{C R}}$ in the display preceding Result 1.5. The pairs of the first kind are invariant under the action, since it is by unary endomorphisms. The same is also true for the pairs of the second kind, since ${ }^{t_{1}}\left((t, y)^{\prime}\right)=\left({ }^{t_{1}}(t, y)\right)^{\prime}$ for all $t_{1} \in T,(t, y) \in \overline{E G}$. Hence $\rho_{\mathbf{C R}}$ is invariant under the action. Since $\rho_{\mathbf{U}} / \rho_{\mathbf{C R}}$ is fully invariant on $E F \mathbf{C R}_{E G}, \rho_{\mathbf{U}}$ is also invariant under the action.

Now $T$ also acts on $V G=T$ by left translation, and the action on $\overline{E G}=E \bar{G}$ respects heads and tails of edges. Thus $T$ acts on the graph $\bar{G}$ and, by extension, acts by unary endomorphisms on $G^{u}$. It is clear that the action is left unitary, transitive and fixed point free. Referring to $\S 7.2$, we may therefore construct the semigroup $G^{u} / T$ of orbits of $G^{u}$, under the action of $T$, and with the obvious choice of 1 as distinguished vertex, $G^{u} / T \cong S_{1}\left(G^{u}\right)$. A pleasant notational simplification is that for any vertex $t, g_{t}$ turns out to be just $t$ itself.

Now it is also clear that the action is compatible with $\eta$, in the sense that for any $t \in T$ and any unary path $p$ over $\bar{G},\left({ }^{t} p\right) \eta={ }^{t}(p \eta)$. Hence the congruence $\tau_{\mathbf{U}}$ on $G^{u}$ induced from $\rho_{\mathbf{U}}$ is also invariant under the action and, referring again to $\S 7.2, T$ acts on $G^{u} / \tau_{\mathbf{U}}$ and the induced faithful morphism $\bar{\eta}: G^{u} / \tau_{\mathbf{U}} \rightarrow(E G)^{u} / \rho_{\mathbf{U}}$ is compatible with this action. We may therefore also construct the semigroup $\left(G^{u} / \tau_{\mathbf{U}}\right) / T$, which is isomorphic to $S_{1}\left(G^{u} / \tau_{\mathbf{U}}\right)$ and to $S_{1}\left(G^{u}\right) /\left(\tau_{\mathbf{U}}\right)_{1}$.

We now show that these semigroups are isomorphic to the e-free semigroup on $X$ in $\mathbf{U} * \mathbf{V}$, by proving directly that the latter is isomorphic to $S_{1}\left(G^{u} / \tau_{\mathbf{U}}\right)$. Let $j: \bar{X} \rightarrow S_{1}\left(G^{u} / \tau_{\mathbf{U}}\right)$ be given by $y j=(1, y) \tau_{\mathbf{U}}, y \in \bar{X}$ (interpreting $y$ in $T$ as either $x$ or $x^{-1}$, as earlier).

Theorem 7.4. Let $\mathbf{U}$ be an e-variety of completely regular semigroups, $\mathbf{V}$ a variety of groups and $X$ a nonempty set. Put $T=F \mathbf{V}_{X}$, let $G$ be the Cayley graph of $T$, and let $G^{u}$ be the free unary semigroupoid on the graph $\bar{G}$ (where we identify $E \bar{G}$ with $\overline{E G}$ and with $T \times \bar{X})$. Let $\tau_{\mathbf{U}}$ be the congruence on $G^{u}$ induced by the least $\mathbf{U}$-congruence $\rho_{\mathbf{U}}$ on the free unary semigroup $(E G)^{u}$.

Then $E F(\mathbf{U} * \mathbf{V})_{X}$ is isomorphic to the semigroup $\left(G^{u} / \tau_{\mathbf{U}}\right) / T$ of orbits of $G^{u} / \tau_{\mathbf{U}}$ under the action of $T$; also to the semigroup $S_{1}\left(G^{u}\right) /\left(\tau_{\mathbf{U}}\right)_{1}$, where $S_{1}\left(G^{u}\right)=$ $\bigcup_{t \in T} G^{u}(1, t)$, with product $p \star q=p^{h(p)} q$, and $\left(\tau_{\mathbf{U}}\right)_{1}$ is the restriction of $\tau_{\mathbf{U}}$ to $S_{1}\left(G^{u}\right)$; and to the semigroup $S_{1}\left(G^{u} / \tau_{\mathbf{U}}\right)$, defined similarly.

Proof. We use freely the notation and terminology introduced above. The proof follows in outline that of Theorem 6.3 , showing that $S_{1}\left(G^{u} / \tau_{\mathbf{U}}\right)$ is isomorphic to the subsemigroup $\langle\bar{X} i\rangle_{e}$ of $S * T$ described in Theorem 7.1.

Define $\psi: S_{1}\left(G^{u} / \tau_{\mathbf{U}}\right) \rightarrow S * T$ by $p \psi=(p \bar{\eta}, h(p))$. Now

$$
\begin{aligned}
(p \star q) \psi & =\left(p^{h(p)} q\right) \psi=\left(\left(p^{h(p)} q\right) \bar{\eta}, h(p \star q)\right)=\left(p \bar{\eta}\left({ }^{h(p)} q\right) \bar{\eta}, h(p) h(q)\right) \\
& =\left(p \bar{\eta}^{h(p)} q \bar{\eta}, h(p) h(q)\right)=(p \bar{\eta}, h(p))(q \bar{\eta}, h(q))=p \psi q \psi
\end{aligned}
$$

so $\psi$ is a morphism. The proof that $\psi$ is injective proceeds exactly as in the proof of Theorem 6.3. 
For $y \in \bar{X}, y j \psi=(1, y) \tau_{\mathbf{U}} \psi=\left((1, y) \tau_{\mathbf{U}} \bar{\eta}, y\right)=\left((1, y) \eta \rho_{\mathbf{U}}, y\right)=((1, y), y)=y i$ (recalling that $S$ is assumed to contain $T \times \bar{X})$. By Theorem 7.1 it remains to show that $S_{1}\left(G^{u} / \tau_{\mathbf{U}}\right)$ is regular and equals $\left\langle(1, y) \tau_{\mathbf{U}}: y \in \bar{X}\right\rangle_{e}$. We need some facts from [14].

Let $\sim$ be the unary congruence on $G^{u}$ generated by the pairs $\left(p(p)^{-1} p, p\right)$, $\left(p(p)^{-1},(p)^{-1} p\right),\left(\left((p)^{-1}\right)^{-1}, p\right)$, for all loops $p$, together with the pairs $\left(e e^{\prime} e, e\right)$, for all edges $e=(t, y) \in E \bar{G}$. Then ([14, Proposition 7]) $G^{u} / \sim$ is a regular, locally completely regular semigroupoid. (In fact, it is isomorphic to $E F \mathbf{l C R}_{G}$.) It is easily seen (cf. the beginning of $\S 4$ of [14]) that $\sim$ is contained in $\tau_{\mathbf{C R}}$ and is therefore contained in $\tau_{\mathbf{U}}$. Hence $G^{u} / \tau_{\mathbf{U}}$ is also a regular, locally completely regular semigroupoid.

Now let $\pi^{\prime}: G^{u} / \tau_{\mathbf{U}} \rightarrow\left(G^{u} / \tau_{\mathbf{U}}\right) / T$ be the unary morphism that maps each edge $p$ of $G^{u} / \tau_{\mathbf{U}}$ to its orbit $[p]$ (see $\S 7.2$ ). Since $\pi^{\prime}$ is surjective, in the obvious sense, $\left(G^{u} / \tau_{\mathbf{U}}\right) / T$ is a regular semigroup. Further, the image of each local semigroup of $G^{u} / \tau_{\mathbf{U}}$ is a completely regular subsemigroup.

Let $R$ be any regular subsemigroup of $\left(G^{u} / \tau_{\mathbf{U}}\right) / T$ that contains $\left\{\left[(1, y) \tau_{\mathbf{U}}\right]\right.$ : $y \in \bar{X}\}$. We use the recursive definition of $G^{u}$ to show that $R$ contains $\left[p \tau_{\mathbf{U}}\right]$ for every edge $p \in E G^{u}$. First, for any edge $(t, y) \in E \bar{G},\left[(t, y) \tau_{\mathbf{U}}\right]=\left[(1, y) \tau_{\mathbf{U}}\right] \in R$. Next, if $p$ and $q$ are consecutive edges of $G^{u}$ such that $\left[p \tau_{\mathbf{U}}\right],\left[q \tau_{\mathbf{U}}\right] \in R$, then $\left[(p q) \tau_{\mathbf{U}}\right]=\left[p \tau_{\mathbf{U}} q \tau_{\mathbf{U}}\right]=\left[p \tau_{\mathbf{U}}\right]\left[q \tau_{\mathbf{U}}\right] \in R$. Finally, if $p$ is a loop of $G^{u}$ such that $\left[p \tau_{\mathbf{U}}\right] \in R$, then $\left[p \tau_{\mathbf{U}}\right]$ belongs to a completely regular subsemigroup of $R$, so that $\left(\left[p \tau_{\mathbf{U}}\right]\right)^{-1} \in R$. But since $\tau_{\mathbf{U}}$ is a unary congruence and $\pi^{\prime}$ is a unary morphism, $\left(\left[p \tau_{\mathbf{U}}\right]\right)^{-1}=\left[(p)^{-1} \tau_{\mathbf{U}}\right]$. Thus $\left(G^{u} / \tau_{\mathbf{U}}\right) / T=\left\langle\left[(1, y) \tau_{\mathbf{U}}\right]: y \in \bar{X}\right\rangle_{e}$.

Finally, under the isomorphism $\left(G^{u} / \tau_{\mathbf{U}}\right) / T \rightarrow S_{1}\left(G^{u} / \tau_{\mathbf{U}}\right)$ of $\S 7.2,\left[p \tau_{\mathbf{U}}\right]$ is mapped to $p \tau_{\mathbf{U}} \pi={ }^{t(p)} p \tau_{\mathbf{U}}$, for any unary path $p$, whence in particular $\left[(1, y) \tau_{\mathbf{U}}\right]$ is mapped to $(1, y) \tau_{\mathbf{U}}$. Hence $S_{1}\left(G^{u} / \tau_{\mathbf{U}}\right)$ has the requisite properties.

The extra information conferred by this theorem, when compared with that of $\S 7.1$, is that $E F(\mathbf{U} * \mathbf{V})_{X}$ consists of all the $\tau_{\mathbf{U}}$-classes of the unary paths over $\bar{G}$ that start at 1 . If the e-free completely regular semigroups in $\mathbf{U}$ and the free groups in $\mathbf{V}$ have a solvable word problem (for $\mathbf{C R}$ and $\mathbf{G}$, for instance), then membership of classes of paths can be decided.

Our theorem also encompasses the construction of the e-free semigroups in the e-varieties $\mathbf{A} * \mathbf{V}=\mathbf{A m} \mathbf{V}$, where $\mathbf{A}$ is any variety of bands (and $\mathbf{V}$ is any variety of groups). These semigroups were first constructed by Kaďourek and Szendrei [19, Theorem 6.2] and re-interpreted in the manner of the theorem above in [34, Result 1.6]. Since, when considered as unary semigroups, the unary operation on bands is just the identity map, we may first factor $G^{u}$ by the congruence that identifies each loop with its inverse, before applying $\tau_{\mathbf{A}}$ (more precisely the congruence that it induces); the factor semigroupoid is then simply the free semigroupoid on $\bar{G}$, which may be interpreted, as in [19], as the free involutory semigroupoid on $G$. A similar treatment can be applied to $(E G)^{u}$, yielding the free involutory semigroup on $E G$. We then obtain exactly the second construction cited above.

\section{E-FREE SEMIGROUPS IN E-VARIETIES $\mathbf{I} * \mathbf{V}$}

We describe the e-free semigroups, whenever they exist, in the e-varieties of the form $\mathbf{U} * \mathbf{V}$, where $\mathbf{U}$ is a variety of inverse semigroups and $\mathbf{V}$ is any e-variety. By Propositions 3.11 and 3.14, such products fall into two classes: (1) those where $\mathbf{V} \subseteq \mathbf{C S}$, in which case $\mathbf{U} * \mathbf{V} \subseteq L \mathbf{I}$, and (2) those where $\mathbf{U} \subseteq \mathbf{G}$ and $\mathbf{V} \subseteq \mathbf{E S}$, 
in which case $\mathbf{U} * \mathbf{V} \subseteq \mathbf{E S}$. (We need not consider the products $\mathbf{T} * \mathbf{V}=\mathbf{V}$, $\mathbf{V} \subseteq L \mathbf{I}$.) In $\S 8.1$ we use Proposition 3.6 and Theorem 4.5 to specialize the general result to obtain new descriptions of e-free semigroups in $L \mathbf{I}$ and certain of its sub-evarieties. In $\S 8.2$ we likewise use Theorem 5.5 to obtain new descriptions of the e-free semigroups in certain important (e-) varieties of completely regular semigroups; by a simple modification we also obtain the free (unary) semigroups in those varieties. Since the argument will parallel closely that for $E$-solid semigroups, we shall pass over some of the details. At this point only the 'semigroup-theoretic' approach has proved feasible to the authors. An analogue of the 'graph-theoretic' approach might allow refinement of our solution in the manner of the preceding sections.

Let $\mathbf{U}$ be any variety of inverse semigroups and let $\mathbf{V}$, as above, be an $E$ solid e-variety that is contained in $\mathbf{C S}$ if $\mathbf{U}$ does not consist entirely of groups. Let $X$ be a nonempty set and let $X^{\prime}$ and $\bar{X}$ be as in $\S 1$. For convenience, put $T=E F \mathbf{V}_{X}$. Let $S=F \mathbf{U}_{T^{1} \times \bar{X}}$. Recall that e-varieties of inverse semigroups are simply the varieties of such semigroups, considered as unary semigroups; and thus e-free inverse semigroups are simply (relatively) free inverse semigroups in the usual sense. We may regard $S$ as being generated, as a unary semigroup, by $T^{1} \times \bar{X}$ itself.

Let $T$ act on $T^{1} \times \bar{X}$ by ${ }^{t_{1}}(t, y)=\left(t_{1} t, y\right)$, for $t_{1} \in T, t \in T^{1}, y \in \bar{X}$. As in the previous section, this action extends to an action of $T$ on $S$, and a semidirect product $S * T$ is defined. By Theorem 3.1, $\operatorname{Reg}(S * T)$ is a (regular) subsemigroup of $S * T$ and so belongs to $\mathbf{U} * \mathbf{V}$.

Define $i: \bar{X} \rightarrow S * T$ by $x i=\left((1, x)\left(x x^{\prime}, x\right)^{-1}\left(x x^{\prime}, x\right)\left(x, x^{\prime}\right)\left(x, x^{\prime}\right)^{-1}, x\right)$ and $x^{\prime} i=\left(\left(1, x^{\prime}\right)\left(x^{\prime} x, x^{\prime}\right)^{-1}\left(x^{\prime}, x\right)^{-1}, x^{\prime}\right)$, for $x \in X$.

Theorem 8.1. Let $\mathbf{U}$ be a variety of inverse semigroups, let $\mathbf{V}$ be an e-variety of E-solid semigroups that is contained within $\mathbf{C S}$ if $\mathbf{U}$ does not consist entirely of groups, and let $X$ be a nonempty set. Let $E F \mathbf{V}_{X}$ act on $F \mathbf{U}_{E F \mathbf{V}_{X}^{1} \times \bar{X}}$ as above. Then $E F(\mathbf{U} * \mathbf{V})_{X}$ is isomorphic to the regular subsemigroup of $F \mathbf{U}_{E F \mathbf{V}_{X}^{1} \times \bar{X}} *$ $E F \mathbf{V}_{X}$ generated by

$$
\begin{gathered}
\left\{\left((1, x)\left(x x^{\prime}, x\right)^{-1}\left(x x^{\prime}, x\right)\left(x, x^{\prime}\right)\left(x, x^{\prime}\right)^{-1}, x\right): x \in X\right\} \\
\cup\left\{\left(\left(1, x^{\prime}\right)\left(x^{\prime} x, x^{\prime}\right)^{-1}\left(x^{\prime}, x\right)^{-1}, x^{\prime}\right): x \in X\right\} .
\end{gathered}
$$

Proof. We use the notation $S$ and $T$ introduced above. By Theorem 3.1 and Propositions 3.11 and $3.14, \operatorname{Reg}(S * T) \leq S * T$ and $\operatorname{Reg}(S * T)$ belongs to $L \mathbf{I}$ or to ES. A straightforward computation, using commutativity of idempotents in $S$, verifies that for any $x \in X, x^{\prime} i \in V(x i)$; that is, $i$ is a matched map. By Result 1.6, the given subsemigroup, namely $\langle\bar{X} i\rangle_{e}$, is well defined.

Now let $U \in \mathbf{U}$ and $V \in \mathbf{V}$, with $V$ acting on $U$, left unitarily if $V$ is a monoid. Extend the action to one of $V^{1}$ on $U$ by letting 1 act identically. Suppose $\phi$ : $\bar{X} \rightarrow U * V$ is a matched mapping. For each $y \in \bar{X}$, put $y \phi=\left(y_{U}, y_{V}\right)$. The equations $x_{U}{ }^{x_{V}} x_{U}^{\prime}{ }^{x_{V} x_{V}^{\prime}} x_{U}=x_{U}$ and $x_{U}^{\prime}{ }^{x_{V}^{\prime}} x_{U}{ }^{x_{V}^{\prime} x_{V}} x_{U}^{\prime}=x_{U}^{\prime}$ hold for any $x \in X$. From the uniqueness of inverses in $S$ it follows that ${ }^{x_{V}^{\prime} x_{V}} x_{U}^{\prime}=\left({ }^{x_{V}^{\prime}} x_{U}\right)^{-1}$ and ${ }^{x_{V} x_{V}^{\prime}} x_{U}=\left({ }^{x_{V}} x_{U}^{\prime}\right)^{-1}$.

Define $\beta: T \rightarrow V$ to be the unique extension to $T$ of the matched map $y \rightarrow y_{V}$, $\bar{X} \rightarrow V$. Also denote by $\beta$ its extension to a morphism $T^{1} \rightarrow V^{1}$, where $1 \beta=1$. Next define $\alpha: T^{1} \times \bar{X} \rightarrow U$ by $(t, y) \alpha={ }^{t \beta} y_{U}$, for all $t \in T^{1}, y \in \bar{X}$. Since $S$ is free as a unary semigroup, $\alpha$ extends to a morphism (automatically unary), again denoted $\alpha$, from $S$ to $U$. It is again easily verified that $\left({ }^{t} s\right) \alpha={ }^{t \beta} s \alpha$ for all $t \in T^{1}, s \in S$. Hence the product map $\theta=\alpha \times \beta: S * T \rightarrow U * V$ is a morphism. 
Using the equations above we obtain

$$
\begin{aligned}
x i \theta & =\left(x_{U}\left({ }^{x_{V} x_{V}^{\prime}} x_{U}\right)^{-1 x_{V} x_{V}^{\prime}} x_{U}{ }^{x_{V}} x_{U}^{\prime}\left({ }^{x_{V}} x_{U}^{\prime}\right)^{-1}, x_{V}\right) \\
& =\left(x_{U}{ }^{x_{V}} x_{U}^{\prime}{ }^{x_{V} x_{V}^{\prime}} x_{U}{ }^{x_{V}} x_{U}^{\prime}{ }^{x_{V} x_{V}^{\prime}} x_{U}, x_{V}\right) \\
& =\left(x_{U}, x_{V}\right)
\end{aligned}
$$

and, similarly,

$$
\begin{aligned}
x^{\prime} i \theta & =\left(x_{U}^{\prime}\left(x_{V}^{\prime} x_{V} x_{U}^{\prime}\right)^{-1}\left({ }^{x_{V}^{\prime}} x_{U}\right)^{-1}, x_{V}^{\prime}\right) \\
& =\left(x_{U}^{\prime}, x_{V}^{\prime}\right) .
\end{aligned}
$$

Hence $i \theta=\phi$. Now an application of Lemma 7.2 completes the proof, as in the proof of Theorem 7.1.

8.1. e-free locally inverse semigroups. In the situation where $\mathbf{U} \subseteq \mathbf{I}$ and $\mathbf{V} \subseteq$ $\mathbf{C S}$, we have $\mathbf{U} * \mathbf{V} \subseteq L \mathbf{I}$, as remarked above. By Theorem $4.5, \mathbf{I} * \mathbf{R Z}$ equals $L \mathbf{I}$ itself. Of course the free inverse semigroups are well described [31]. In view of the importance of locally inverse semigroups it is worth stating our description of e-free members separately. The e-free right zero semigroup on $X$ is simply the set $\bar{X}$ itself, under right zero multiplication. Its action on $T^{1} \times \bar{X}=\bar{X}^{1} \times \bar{X}$ is given by ${ }^{t_{1}}(t, y)=(t, y)$ and ${ }^{t_{1}}(1, y)=\left(t_{1}, y\right)$, for $t_{1}, t, y \in \bar{X}$.

Corollary 8.2. Let $X$ be a nonempty set. Let $E F \mathbf{R} \mathbf{Z}_{X}$ act on $F \mathbf{I}_{\bar{X}^{1} \times \bar{X}}$ as above. Then EFLI $\mathbf{I}_{X}$ is isomorphic to the regular subsemigroup of $F \mathbf{I}_{\bar{X}^{1} \times \bar{X}} * E F \mathbf{R Z}_{X}$ generated by

$$
\begin{gathered}
\left\{\left((1, x)\left(x^{\prime}, x\right)^{-1}\left(x^{\prime}, x\right)\left(x, x^{\prime}\right)\left(x, x^{\prime}\right)^{-1}, x\right): x \in X\right\} \\
\cup\left\{\left(\left(1, x^{\prime}\right)\left(x, x^{\prime}\right)^{-1}\left(x^{\prime}, x\right)^{-1}, x^{\prime}\right): x \in X\right\}
\end{gathered}
$$

By expanding on an idea of Yeh [42], Auinger [3, 4] has considered locally inverse semigroups as 'binary semigroups' — semigroups with an additional binary operation (derived from its 'sandwich sets' - for more details see $\$ 10$ ). The elements of e-free locally inverse semigroups may then be considered as 'words' of this type. From this point of view the corollary is easily translated into a solution of the word problem.

Our description bears little resemblance to either of the two descriptions of efree locally inverse semigroups given by Auinger $[3,4]$. This is not at all surprising, since a consequence of his description is a decomposition of $L \mathbf{I}$ as the semidirect product $\mathbf{S} * \mathbf{C S}$, about which we say more below.

Other interesting special cases of Theorem 8.1 in which $\mathbf{V}=\mathbf{R Z}$ include those where $\mathbf{U}=\mathbf{S}$ and $\mathbf{U}=\mathbf{S G}$. Of course, the free semilattices and free Clifford semigroups are also well described. By Proposition 4.6, a slight variation of the corollary yields a description of $E F \mathbf{C S R}_{X}$ alternative to that of Auinger [2], and a description of $E F \mathbf{S R}_{X}$ that is new, to the authors' knowledge. Yet another case of this type is where $\mathbf{U}=\mathbf{G}$. By Theorem 4.5, a description of $E F \mathbf{C S}_{X}$ is obtained. A simple Rees matrix representation for this semigroup is already known [3, Corollary 3.3], however.

Auinger [4, Theorem 4.13] described $E F L \mathbf{I}_{X}$ as a well-determined subset of a semidirect product of a certain free semilattice with the e-free completely simple semigroup on $X$. In addition to the Rees representation of the latter just mentioned, 
Auinger also provides an alternative represention in terms of canonical forms, on which the cited description of the e-free locally inverse semigroups is based. (See also $\S 10.3$.)

Result 8.3. The e-variety LI equals $\mathbf{S} * \mathbf{C S}$.

Yet another description of $E F L \mathbf{I}_{X}$ may therefore also be derived from Theorem 8.1, in terms of $T$, the e-free completely simple semigroup on $X$, and $S$, the free semilattice on $T^{1} \times \bar{X}$. It would be of interest to correlate this description with that of Auinger just cited. On the one hand, the latter certainly has the advantage of prescribing which elements of the semidirect product belong to the e-free semigroup. On the other hand, our theorem also describes the e-free semigroups in $\mathbf{S} * \mathbf{V}$ for any variety $\mathbf{V}$ of completely simple semigroups (without necessarily solving the 'word problem', of course).

8.2. Free and e-free completely regular semigroups. In $\S 5.2$ we showed that if $\mathbf{V}$ is any variety of completely regular semigroups that does not consist entirely of groups, then $\mathbf{G} * \mathbf{V}=\mathbf{L} \mathbf{G m} \mathbf{V}$ and is again a variety of completely regular semigroups. The operator $(-)^{T_{l}}$, which takes a completely regular variety $\mathbf{V}$ to $\mathbf{L} \mathbf{G m} \mathbf{V}$, and its dual $(-)^{T_{r}}$ have played a major role in investigations of the structure of the lattice of completely regular semigroup varieties. See [29] for the major properties of these and related operators, and for relationships between them and the syntactic methods of Polák.

Now it is clear that Theorem 8.1 applies to describe the e-free semigroups in $\mathbf{G} * \mathbf{V}$ as (completely regular) subsemigroups of semidirect products of free groups and e-free semigroups in $\mathbf{V}$. In this case, however, the generators prescribed by the theorem simplify to yield the same form as those prescribed for $E$-solid semigroups in Theorem 7.1. Recall the convention that $(t, x)^{\prime}=\left(t x, x^{\prime}\right)$.

Corollary 8.4. Let $\mathbf{V}$ be a variety of completely regular semigroups and let $X$ be a nonempty set. Then $E F \mathbf{V}_{X}^{T_{l}}$ is isomorphic to the (completely) regular subsemigroup of $F \mathbf{G}_{E F \mathbf{V}_{X}^{1} \times \bar{X}} * E F \mathbf{V}_{X}$ generated by $\{((1, y), y): y \in \bar{X}\}$.

The word problem for e-free semigroups in completely regular varieties may be formulated in terms of deciding equality of pairs of unary words over $\bar{X}$ (that is, pairs of elements of the semigroup $X^{u}$ defined in $\S 1$ ). Recall from the proof of Theorem 5.5 that in any semidirect product $G * T$ with $G$ a group and $T$ completely regular, $(g, t)^{-1}=\left(g\left({ }^{t^{0}} g\right)^{-1}\left(t^{-1} g\right)^{-1}, t^{-1}\right)$. By evaluating pairs of unary words in the semidirect product described in the corollary, we obtain a procedure for deciding their equality. This procedure is effective whenever the word problem for the e-free semigroups in $\mathbf{V}$ is soluble.

Since completely regular semigroup varieties actually possess free semigroups, it is perhaps of more interest to describe them. The proof of the following result involves essentially the same steps as that of Theorems 7.1 and 8.1, so we have omitted it. (In fact, free semigroups in any semidirect product $\mathbf{U} * \mathbf{V}$, where $\mathbf{U} \subseteq \mathbf{C S}$ and $\mathbf{V} \subseteq \mathbf{C R}$, may be constructed in terms of free semigroups in $\mathbf{U}$ and $\mathbf{V}$, via a variation of the methods of these two sections.) The free semigroups in $\mathbf{V}^{T_{r}}$ are constructed dually, using the 'reverse' semidirect product.

Theorem 8.5. Let $\mathbf{V}$ be a variety of completely regular semigroups and let $X$ be a nonempty set. Then $F \mathbf{V}_{X}^{T_{l}}$ is isomorphic to the (completely) regular subsemigroup of $F \mathbf{G}_{F \mathbf{V}_{X}^{1} \times X} * F \mathbf{V}_{X}$ generated by $\{((1, x), x): x \in X\}$. 
Similarly to the remarks following the corollary, the word problem in the free semigroups in $\mathbf{V}^{T_{l}}$ is soluble modulo that in the free semigroups in $\mathbf{V}$. The word problem in $F \mathbf{V}_{X}^{T_{l}}$ was first solved by J. Kadourek [17], modulo that in the free semigroups in $\mathbf{V}$, as a byproduct of his solution to the word problem in $F(\mathbf{G m} \mathbf{V})_{X}$ (see page 7 of the cited paper). Not only does Theorem 8.5 provide a more explicit description of these free semigroups, but the solution to the word problem is considerably more transparent than Kadourek's.

An interesting special case is that of $\mathbf{B}^{T_{l}}$, the variety of bands of left groups. Since the solution to the word problem in free bands is well known, it follows that the word problem is soluble in free bands of left groups. Moreover, all nontrivial subgroups of such completely regular semigroups are free. For in any semidirect product $G * B$, where $G$ is a group and $B$ is a band, if $(g, e)$ is any idempotent, then the map $(h, e) \rightarrow{ }^{e} h$ embeds the $\mathcal{H}$-class of $(g, e)$ in $G$. Thus the Schreier subgroup theorem applies. It is interesting to note that the maximal subgroups in free bands of groups, that is, in the free semigroups in $\mathbf{G m B}$, are not in general free [40].

\section{A Unified CONSTRuCtion OF E-FreE SEMigroups}

We provide here a construction that encompasses all those of the preceding two sections. The three situations covered there were for semidirect products $\mathbf{U} * \mathbf{V}$ where (i) $\mathbf{U} \subseteq \mathbf{E S}$ and $\mathbf{V} \subseteq \mathbf{G}$, or (ii) $\mathbf{U} \subseteq \mathbf{I}$ and $\mathbf{V} \subseteq \mathbf{C S}$, or (iii) $\mathbf{U} \subseteq \mathbf{G}$ and $\mathbf{V} \subseteq \mathbf{E S}$. Note that $\mathbf{U}$ and $\mathbf{V}$ are $E$-solid in all these cases. The reader may discern which semidirect products of $E$-solid e-varieties are not covered by comparison with the results of $\S 3$.

The general result, presented below, is less enlightening than might first appear, for three reasons. Firstly, it covers no situations other than those already treated. Secondly, the description is more complicated than in either of the separate situations. In that of $\S 8$, it reduces immediately to Theorem 8.1 , but in that of $\S 7$, some simplification must be made to reduce to Theorem 7.1. Thirdly, its proof essentially breaks into those already given (with some additional complexity in the situation of $\S 7$ ). It was for these reasons that we have deferred the general presentation until after the specific ones, and have given only a bare outline of the proof.

Let $\mathbf{U}, \mathbf{V}$ be as above, let $X$ be a nonempty set and let $X^{\prime}$ and $\bar{X}$ be as in $\S 1$. Let $T=E F \mathbf{V}_{X}$ and let $S=E F \mathbf{U}_{T^{1} \times \bar{X}}$. (To construct $S$, the set $T^{1} \times \bar{X}$ must also be 'doubled'.)

Now $T$ acts on $T^{1} \times \bar{X}$ by ${ }^{t_{1}}(t, y)=\left(t_{1} t, y\right)$ and on $\left(T^{1} \times \bar{X}\right)^{\prime}$ by ${ }^{t_{1}}(t, y)^{\prime}=$ $\left(t_{1} t, y\right)^{\prime}$, for $t_{1} \in T, t \in T^{1}, y \in \bar{X}$. The action is by matched mappings, so it extends to an action of $T$ on $S$, and a semidirect product $S * T$ is defined. From the assumptions, $\operatorname{Reg}(S * T)$ belongs to $\mathbf{U} * \mathbf{V}$.

Since $S$ is $E$-solid, its core is completely regular. Thus if $e, f \in E_{S}$, we denote by $(e f)^{0}$ the identity element $(e f)(e f)^{-1}=(e f)^{-1}(e f)$ of the maximal subgroup to which $e f$ belongs. Note that since $T$ acts on $S$ by endomorphisms, an action is induced on $C(S)$; the latter being completely regular, the action is therefore compatible with the operations $u \rightarrow u^{-1}$ and $u \rightarrow u^{0}$ on $C(S)$.

Define $i: \bar{X} \rightarrow S * T$ as follows. For $x \in X$, put

$$
e_{x}=\left(\left(x x^{\prime}, x\right)^{\prime}\left(x x^{\prime}, x\right)\left(x, x^{\prime}\right)\left(x, x^{\prime}\right)^{\prime}\right)^{0}
$$

and

$$
f_{x}={ }^{x^{\prime}} e_{x}\left(=\left(\left(x^{\prime}, x\right)^{\prime}\left(x^{\prime}, x\right)\left(x^{\prime} x, x^{\prime}\right)\left(x^{\prime} x, x^{\prime}\right)^{\prime}\right)^{0}\right) .
$$


Then put $x i=\left((1, x) e_{x}, x\right)$ and $x^{\prime} i=\left(\left(1, x^{\prime}\right)\left(x^{\prime} x, x^{\prime}\right)^{\prime} f_{x}\left(x^{\prime}, x\right)^{\prime}, x^{\prime}\right)$.

Theorem 9.1. Let $\mathbf{U}$ and $\mathbf{V}$ be e-varieties of E-solid semigroups such that $\mathbf{V} \subseteq$ $\mathbf{C S}$ whenever $\mathbf{U} \nsubseteq \mathbb{G}$ and, further, $\mathbf{V} \subseteq \mathbf{G}$ whenever $\mathbf{U} \nsubseteq \mathbb{\mathbf { I }}$. Let $X$ be a nonempty set. Let $E F \mathbf{V}_{X}$ act on $E F \mathbf{U}_{E F \mathbf{V}_{X}^{1} \times \bar{X}}$ as above. Then $E F(\mathbf{U} * \mathbf{V})_{X}$ is isomorphic to the regular subsemigroup of $E F \mathbf{U}_{E F \mathbf{V}_{X}^{1} \times \bar{X}} * E F \mathbf{V}_{X}$ generated by

$$
\left\{\left((1, x) e_{x}, x\right): x \in X\right\} \cup\left\{\left(\left(1, x^{\prime}\right)\left(x^{\prime} x, x^{\prime}\right)^{\prime} f_{x}\left(x^{\prime}, x\right)^{\prime}, x^{\prime}\right)\right\},
$$

where $e_{x}$ and $f_{x}$ are as just defined.

Proof. Suppose U, V are as stated. We use the notation $S$ and $T$ introduced above. In the case that $\mathbf{U} \subseteq \mathbf{I}$, the hypotheses reduce to those of Theorem 8.1. In this situation, $S=F \mathbf{U}_{E F \mathbf{V}_{X}^{1} \times X}$ and $(t, y)^{\prime}=(t, y)^{-1}$, for all $t \in T^{1}, y \in \bar{X}$. Using commutativity of idempotents, the prescribed generators reduce to those stated in the same theorem. Hence this case follows from that theorem.

In the remainder of the proof, assume that $\mathbf{V} \subseteq \mathbf{G}$. Then $T=F \mathbf{V}_{X}$. As in the proof of Theorem 7.1, let $U \in \mathbf{U}, V \in \mathbf{V}$, with $V$ acting left unitarily on $U$, suppose $\phi: \bar{X} \rightarrow U * V$ is a matched mapping, and for each $y \in \bar{X}$, put $y \phi=\left(y_{U}, y_{V}\right)$; again, the equations $y_{V}^{\prime}=y_{V}^{-1}$ and $y_{U}{ }^{y_{V}} y_{U}^{\prime} y_{U}=y_{U}$ hold. Define $\beta: T \rightarrow V$ to be the unique extension to $T$ of the map $x \rightarrow x_{V}, X \rightarrow V$. Define $\alpha: \overline{T \times \bar{X}} \rightarrow U$ by $(t, y) \alpha={ }^{t \beta} y_{U}$ and $(t, y)^{\prime} \alpha={ }^{(t y) \beta} y_{U}^{\prime}$, for all $t \in T, y \in \bar{X}$. Then the equations above imply that $(t, y) \alpha \in V\left((t, y)^{\prime} \alpha\right)$. Hence $\alpha$ is matched and extends to a morphism, again denoted $\alpha$, from $S$ to $U$. Again, it is easily verified that the product map $\theta=\alpha \times \beta: S * T \rightarrow U * V$ is a morphism.

After using Lemma 7.2 as in the proof of Theorem 7.1, it remains to show that the map $i: \bar{X} \rightarrow S * T$, defined above, is matched, and that $i \theta=\phi$. Let $x \in X$. Since $T$ is a group, $\left(x x^{\prime}, x\right)=(1, x)$ and $\left(x^{\prime} x, x^{\prime}\right)=\left(1, x^{\prime}\right)$. From the definition of $e_{x}$, it is clear that $e_{x}=e_{x}\left(x, x^{\prime}\right)\left(x, x^{\prime}\right)^{\prime}=(1, x)^{\prime}(1, x) e_{x}$. Hence

$$
\begin{aligned}
& \left((1, x) e_{x}, x\right)\left(\left(1, x^{\prime}\right)\left(1, x^{\prime}\right)^{\prime} f_{x}\left(x^{\prime}, x\right)^{\prime}, x^{\prime}\right)\left((1, x) e_{x}, x\right) \\
& \quad=\left((1, x) e_{x}\left(x, x^{\prime}\right)\left(x, x^{\prime}\right)^{\prime x} f_{x}(1, x)^{\prime}(1, x) e_{x}, x\right) \\
& \quad=\left((1, x) e_{x}\left(x, x^{\prime}\right)\left(x, x^{\prime}\right)^{\prime} e_{x}(1, x)^{\prime}(1, x) e_{x}, x\right) \\
& \quad=\left((1, x) e_{x}, x\right),
\end{aligned}
$$

using the definitions of the partial operation $(-)^{0}$ and of $e_{x}$ itself. Thus we obtain $(x i)\left(x^{\prime} i\right)(x i)=x i$ and, similarly, $\left(x^{\prime} i\right)(x i)\left(x^{\prime} i\right)=x^{\prime} i$, as required. Further,

$$
x i \theta=\left(x_{U}\left({ }^{x_{V}} x_{U}^{\prime} x_{U}{ }^{x_{V}} x_{U}^{\prime} x_{U}\right)^{0}, x_{V}\right)=\left(x_{U}, x_{V}\right)=x \phi
$$

and, similarly, $x^{\prime} i \theta=x^{\prime} \phi$. Hence $i \theta=\phi$.

Finally, observe that $\overline{T^{1} \times \bar{X}}=\left(T^{1} \times X\right) \cup\left(T^{1} \times X^{\prime}\right) \cup\left(T^{1} \times X\right)^{\prime} \cup\left(T^{1} \times X^{\prime}\right)^{\prime}$. In the situation where $T$ is a group, we may extend the identification of $(T \times X)^{\prime}$ with $T \times X^{\prime}$ that was made in $\S 7.1$ to an identification of $\overline{T \times \bar{X}}$ with $T \times \bar{X}$, by similarly identifying $\left(T \times X^{\prime}\right)^{\prime}$ with $T \times X$. The extended identification is via the map $(t, y)^{\prime} \rightarrow\left(t y, y^{\prime}\right), t \in T, y \in \bar{X}$. Under this correspondence, and using the simplifications made during the proof above, we may then recover the statement of Theorem 7.1. 


\section{AN ALTERNATIVE PRODUCT OF REGULAR SEMIGROUPS}

Recently, K. Auinger and L. Polák [5] have defined a product $S \bowtie T$ of regular semigroups $S$ and $T$ that yields a regular semigroup whenever $T$ is locally inverse. There is an associated product $\mathbf{U} \bowtie \mathbf{V}$ of e-varieties $\mathbf{U}$ and $\mathbf{V}$, defined whenever $\mathbf{V} \subseteq L \mathbf{I}$. Their work generalizes the second author's construction of a product of '*_ regular' semigroups, under similar hypotheses [32]; ultimately it derives from certain products of inverse semigroups defined by Cowan and by Billhardt. We show that the 'standard' arguments of our paper also apply to their product, so that the e-free semigroups (of rank two or more) in $\mathbf{U} \bowtie \mathbf{V}$ are constructed whenever they exist. The results of this section, other than Result 10.1 and Theorem 10.5(iii), are new. When both $\mathbf{U}$ and $\mathbf{V}$ are locally inverse, so that $\mathbf{U} \bowtie \mathbf{V}$ also has that property [5], the outcome of our construction is equivalent to that of Auinger and Polák. However, their arguments are syntactic, based on 'words' in free binary semigroups (to which allusion was made in $\$ 8.1$ ), and do not extend to the non-locally inverse situation.

10.1. General properties. Recall that in any regular semigroup $T$, the sandwich set $S(e, f)$ of idempotents $e, f$ is the set $f V(e f) e$. Locally inverse semigroups are characterizable by the property that all such sandwich sets contain exactly one element [27]. Following [42] and [3, 4], the rule $x \wedge y=S\left(y^{\prime} y, x x^{\prime}\right)$, where $y^{\prime} \in V(y)$ and $x^{\prime} \in V(x)$, yields a well defined binary operation on any locally inverse semigroup $T$.

Let $S$ be any semigroup and let $T$ be a locally inverse semigroup acting on $S$. The underlying set of the product $S \bowtie T$ is $\left\{(s, t) \in S \times T:{ }^{t t^{\prime}} s=s\right.$ for some $\left.t^{\prime} \in V(t)\right\}$. It was shown in [5] that $S \bowtie T=\left\{(s, t) \in S \times T:{ }^{t t^{\prime}} s=s\right.$ for all $\left.t^{\prime} \in V(t)\right\}$. Define a product on the set $S \bowtie T$ by

$$
(x, u)(y, v)=\left({ }^{u(v \wedge u) u^{\prime}} x^{u} y, u v\right),
$$

where $u^{\prime} \in V(u)$. According to [5], the product is independent of the choice of inverse for $u$. Readers uncomfortable with the $\wedge$ operation may prefer the cumbersome notation $v v^{\prime}\left(u^{\prime} u v v^{\prime}\right)^{\prime} u^{\prime} u$ to $v \wedge u$; here ' indicates taking an arbitrary inverse of the indicated element.

Result 10.1. Let $S, T$ be semigroups, with $T$ locally inverse and acting on $S$. Then

(i) $S \bowtie T$ is a semigroup;

(ii) $E_{S \bowtie T}=\left\{(e, f) \in S \bowtie T: e \in E_{S}, f \in E_{T}\right\}$;

(iii) for $(x, y) \in S \bowtie T, V(x, y)=\left\{\left({ }^{y^{\prime}} x^{\prime}, y^{\prime}\right): x^{\prime} \in V(x), y^{\prime} \in V(y)\right\}$;

(iv) if $S$ is regular, then $S \bowtie T$ is regular;

(v) if $S$ is locally inverse, then so is $S \bowtie T$.

Note that $S \bowtie T$ is not in general a subsemigroup of $S * T$. (See, however, $\S 10.3$.)

If $\mathbf{U}, \mathbf{V}$ are e-varieties of regular semigroups, with $\mathbf{V} \subseteq L \mathbf{I}$, let $\mathbf{U} \bowtie \mathbf{V}$ be the e-variety generated by $\{S \bowtie T: S \in \mathbf{U}, T \in \mathbf{V}\}$.

For any semigroup $S$ and locally inverse semigroup $T$, we define $S \bowtie_{\text {wr }} T$ to be the semigroup that results from the above construction when applied to $S^{T}$ and $T$, where for $\alpha \in S^{T}$ and $t \in T,{ }^{t} \alpha$ is given by $u\left({ }^{t} \alpha\right)=(u t) \alpha, u \in T$. (In contrast with the wreath product construction in $\S 1$, it suffices here to use $S^{T}$ rather than $S^{T^{1}}$.) In parallel with Proposition 3.3 we have the following. 
Proposition 10.2. Let $\mathbf{U}, \mathbf{V}$ be e-varieties of regular semigroups, with $\mathbf{V} \subseteq L \mathbf{I}$. Then $\mathbf{U} \bowtie \mathbf{V}$ is generated by $\left\{S \bowtie_{\mathrm{wr}}, T: S \in \mathbf{U}, T \in \mathbf{V}\right\}$.

Proof. Let $S \in \mathbf{U}, T \in \mathbf{V}$. Define $\theta: S \bowtie T \rightarrow S \bowtie_{\text {wr }} T$ by $(x, u) \theta=\left(\alpha_{x}, u\right)$, where $t \alpha_{x}={ }^{t} x$ for $t \in T$. It is routinely verified that $\theta$ is a well defined morphism. Suppose $(x, u) \theta=(y, v) \theta$. Then $u=v$ and $\alpha_{x}=\alpha_{y}$, whence $x={ }^{u u^{\prime}} x=\left(u u^{\prime}\right) \alpha_{x}=$ $\left(u u^{\prime}\right) \alpha_{y}=u u^{\prime} y=y$, for some inverse $u^{\prime}$ of $u$. Hence $\theta$ is an embedding.

In contrast with $\S 3$, we do not require the action to be left unitary when the second factor is a monoid. However, we may assume so, when convenient, since the action in $S \bowtie_{\mathrm{wr}} T$ is left unitary when $T$ is a monoid.

Similarly to the results of $\S 3$, if $\mathbf{U} \bowtie \mathbf{V}$ lies within either $L \mathbf{I}$ or $\mathbf{E S}$, it consists of the regular semigroups that divide such a product. We now determine when this occurs (cf. Propositions 3.11 and 3.14).

Proposition 10.3. Let $\mathbf{U}, \mathbf{V}$ be e-varieties of regular semigroups, with $\mathbf{V} \subseteq L \mathbf{I}$. Then

(a) $\mathbf{U} \bowtie \mathbf{V} \subseteq L \mathbf{I}$ if and only if $\mathbf{U} \subseteq L \mathbf{I}$, and

(b) $\mathbf{U} \bowtie \mathbf{V} \subseteq \mathbf{E S}$ if and only if either $\mathbf{U} \subseteq \mathbf{C S}$ and $\mathbf{V} \subseteq \mathbf{E S}$, or $\mathbf{U} \subseteq \mathbf{E S}$ and $\mathbf{V} \subseteq \mathbf{G I}$.

Proof. (a) This is immediate from (v) of the previous result.

(b) It was shown in [5] that $\mathbf{C S} \bowtie \mathbf{C S} \subseteq \mathbf{C S}$. As in the proof of Lemma 3.12, by decomposing any completely regular semigroup as a union of its $\mathcal{D}$-classes, we obtain $\mathbf{C S} \bowtie(\mathbf{C R} \cap L \mathbf{I}) \subseteq \mathbf{C R}$; and, similarly to Proposition 3.8, $C(S \bowtie T) \leq S \bowtie C(T)$ for any regular semigroup $S$ and locally inverse semigroup $T$, from which we then obtain $\mathbf{C S} \bowtie(\mathbf{E S} \cap L \mathbf{I}) \subseteq \mathbf{E S}$.

Now suppose that $S \in \mathbf{E S}$ and $T \in \mathbf{G I}$. (Since $T$ is locally inverse, this is equivalent to the hypothesis that $T$ be orthodox). Let $(x, e),(y, f) \in E_{S \bowtie T}$. Then $x, y \in E_{S}, e, f \in E_{T}$ and ${ }^{e} x=x,{ }^{f} y=y$. Since $T$ is orthodox, ef $\in E_{T}$. The calculations involved in computing products may be simplified by choosing $g^{\prime}=g$, for any idempotent $g$. Then $(x, e)(y, f)=\left({ }^{e f} x^{e} y, e f\right)$. Since $S$ is $E$-solid, ${ }^{e f} x^{e} y$ belongs to a subgroup, with inverse $\left({ }^{e f} x^{e} y\right)^{-1}$, following the notational conventions of $\S 9$. From (iii) of the above Result, $\left({ }^{e f}\left({ }^{e f} x^{e} y\right)^{-1}, e f\right) \in V\left({ }^{e f} x^{e} y, e f\right)$. Using the fact that ${ }^{e f}\left({ }^{e f} x^{e} y\right)={ }^{e f} x^{e} y$, it is routinely checked that $\left({ }^{e f}\left({ }^{e f} x^{e} y\right)^{-1}, e f\right)$ commutes with $\left({ }^{e f} x^{e} y, e f\right)$. Thus $(x, e)(y, f)$ belongs to a subgroup of $S \bowtie T$. Hence $S \bowtie T$ is $E$-solid, completing the proof of sufficiency.

Conversely, it is clear that $\mathbf{U}, \mathbf{V}$ must be contained in ES. Suppose $\mathbf{V} \nsubseteq \mathbf{G I}$, that is, $\mathbf{V}$ contains a non-orthodox, $E$-solid semigroup $V$, say. Then $C(V)$ is a non-orthodox completely regular semigroup in $\mathbf{V}$ and, further, some $\mathcal{D}$-class $T$ of $C(V)$ is a non-orthodox completely simple semigroup in $\mathbf{V}$. More specifically, $T$ contains idempotents $e, f$ such that $e f \notin E_{T}$. Also suppose $\mathbf{U} \nsubseteq \mathbb{C S}$. Thus $\mathbf{U}$ contains a two-element semilattice $S=\{0,1\}, 0<1$. We shall show that $S \bowtie_{\text {wr }} T$ is not $E$-solid. Let $\alpha: T \rightarrow S$ be any map such that ${ }^{e} \alpha=\alpha$ and $(e f) \alpha \neq$ $(e f)^{2} \alpha$. (Since $L_{e f} \neq L_{e}$, there is no conflict between these requirements.) Let 1 denote the identity map in $S^{T}$. Then $(\alpha, e),(1, f)$ are idempotents of $S \bowtie_{\mathrm{wr}} T$ and $(\alpha, e)(1, f)=\left({\left({ }^{e f}\right)^{0}}_{\alpha} \alpha, e f\right)$. Observe that ${ }^{e f} \alpha \neq(e f)^{0} \alpha$, since, for example, $(e f)\left({ }^{e f} \alpha\right)=(e f)^{2} \alpha \neq(e f) \alpha=(e f)\left(\left(^{(e f)^{0}} \alpha\right)\right.$. That $(\alpha, e)(1, f)$ cannot belong to a subgroup then follows from the following: 
Lemma 10.4. Let $S$ be a semilattice and $T$ an E-solid locally inverse semigroup that acts on $S$. If $(x, y)$ belongs to a subgroup of $S \bowtie T$ then ${ }^{y} x=y^{0} x$.

Proof. The element $(x, y)$ has an inverse with which it commutes. By (iii) of the above Result, such an inverse has the form $\left({y^{-1}}^{-1}, y^{-1}\right)$. Now $(x, y)\left(y^{-1} x, y^{-1}\right)=$ $\left({ }^{y^{0}} x y^{0} x, y^{0}\right)=\left({ }^{y^{0}} x, y^{0}\right)$, while $\left({ }^{y^{-1}} x, y^{-1}\right)(x, y)=\left({ }^{y^{-1}} x, y^{0}\right)$. Thus ${ }^{y^{0}} x=y^{-1} x$, from which the desired equation follows.

This also completes the proof of the proposition.

Note that if $T$ is a group that acts left unitarily on $S$, then $S \bowtie T$ is just $S * T$ itself. Since we have seen that if $S$ is regular, then $S * T$ is in this case also regular, $S \bowtie T$ coincides with $\operatorname{Reg}(S * T)$. Hence, in view of the remarks following Proposition 10.2, if $\mathbf{V}$ is a variety of groups and $\mathbf{U}$ is an e-variety of regular semigroups, then $\mathbf{U} \bowtie \mathbf{V}=\mathbf{U} * \mathbf{V}$, and the results of $\S 5.1$ apply.

Theorem 10.5. The following equations hold:

(i) $C_{\infty} \mathbf{C R}(\mathbf{H})=\mathbf{C R}(\mathbf{H}) \bowtie \mathbf{G}$, for all group varieties $\mathbf{H}$;

(ii) in particular, $\mathbf{E S}=\mathbf{C R} \bowtie \mathbf{G}$ and $\mathbf{O}=\mathbf{B} \bowtie \mathbf{G}$;

(iii) $L \mathbf{I}=\mathbf{S} \bowtie \mathbf{C S}$.

Proof. The first two statements follow from the previous paragraph, in conjunction with Theorem 5.2. The third can be deduced from the structure theorem for $E F L \mathbf{I}_{X}$ given in [4].

We shall consider the relationship between the two products further in $\S 10.3$.

10.2. e-free semigroups. Let $\mathbf{U}, \mathbf{V}$ be e-varieties of regular semigroups, with $\mathbf{V} \subseteq$ $L \mathbf{I}$, such that $\mathbf{U} \bowtie \mathbf{V}$ is contained in $L \mathbf{I}$ or in ES, as prescribed by Proposition 10.3. We describe the e-free semigroups in $\mathbf{U} \bowtie \mathbf{V}$, in a similar fashion to $\S 9$. (However the arguments most closely parallel those of $\S 7.1$.)

Let $X$ be a nonempty set and let $X^{\prime}$ and $\bar{X}$ be as in $\S 1$. Let $T=E F \mathbf{V}_{X}$ and let $S=E F \mathbf{U}_{T \times X}$. (We form $(T \times X)^{\prime}$ and $\overline{T \times X}$ as in $\S 7.1$, but do not make the identifications that were made there.) Now $T$ acts on $T \times X$ by ${ }^{t_{1}}(t, x)=\left(t_{1} t, x\right)$ and on $(T \times X)^{\prime}$ by ${ }^{t_{1}}(t, x)^{\prime}=\left(t_{1} t, x\right)^{\prime}$. The action is by matched mappings, so it extends to an action of $T$ on $S$ and $S \bowtie T$ is defined and belongs to $\mathbf{U} \bowtie \mathbf{V}$ (cf. the second paragraph of $\S 7.1)$.

Define $i: \bar{X} \rightarrow S \bowtie T$ by $x i=\left(\left(x x^{\prime}, x\right), x\right)$ and $x^{\prime} i=\left(\left(x^{\prime}, x\right)^{\prime}, x^{\prime}\right)$, for $x \in X$. Since ${ }^{x x^{\prime}}\left(x x^{\prime}, x\right)=\left(x x^{\prime}, x\right)$ and ${ }^{x^{\prime} x}\left(x^{\prime}, x\right)^{\prime}=\left(x^{\prime}, x\right)^{\prime}$ for all $x \in X, i$ is well defined.

Theorem 10.6. Let $\mathbf{U}$ and $\mathbf{V}$ be e-varieties of regular semigroups, with $\mathbf{V} \subseteq L \mathbf{I}$ and either $\mathbf{U} \subseteq L \mathbf{I}$, or $\mathbf{U} \subseteq \mathbf{C S}$ and $\mathbf{V} \subseteq \mathbf{E S}$, or $\mathbf{U} \subseteq \mathbf{E S}$ and $\mathbf{V} \subseteq$ GI. Let $X$ be a nonempty set. Let $E F \mathbf{V}_{X}$ act on $E F \mathbf{U}_{E F \mathbf{V}_{X} \times X}$ as above. Then $E F(\mathbf{U} \bowtie \mathbf{V})_{X}$ is isomorphic to the regular subsemigroup of $E F \mathbf{U}_{E F \mathbf{V}_{X} \times X} \bowtie E F \mathbf{V}_{X}$ generated by $\left\{\left(\left(x x^{\prime}, x\right), x\right): x \in X\right\} \cup\left\{\left(\left(x^{\prime}, x\right)^{\prime}, x^{\prime}\right): x \in X\right\}$.

Proof. Let $\mathbf{U}, \mathbf{V}$ be as given and let $S$ and $T$ be as above. By Proposition 10.3, $E F(\mathbf{U} \bowtie \mathbf{V})_{X}$ exists. As in the proof of Theorem 7.1, let $U \in \mathbf{U}, V \in \mathbf{V}$, with $V$ acting on $U$, suppose $\phi: \bar{X} \rightarrow U \bowtie V$ is a matched mapping, and for each $y \in \bar{X}$, put $y \phi=\left(y_{U}, y_{V}\right)$. For each $y \in \bar{X}$, it is clear that $y_{V}^{\prime} \in V\left(y_{V}\right)$, so by the definition of $U \bowtie V, y_{V} y_{V}^{\prime} y_{U}=y_{U}$ and, since $\phi$ is matched, the equation $y_{U}{ }^{y_{V}} y_{U}^{\prime} y_{U}=y_{U}$ holds. 
Define $\beta: T \rightarrow V$ to be the unique extension to $T$ of the matched map $y \rightarrow$ $y_{V}, y \in \bar{X}$. Define $\alpha: \overline{T \times X} \rightarrow U$ by $(t, x) \alpha={ }^{t \beta} x_{U}$ and $(t, x)^{\prime} \alpha={ }^{(t x) \beta} x_{U}^{\prime}$, for $t \in T, x \in X$ (cf. the analogous point in the proof of Theorem 9.1). The equations above imply that $\alpha$ is a matched mapping, whence it extends to a morphism, again denoted $\alpha$, from $S$ to $U$. Again, it is easily verified that $\left({ }^{t} s\right) \alpha={ }^{t \beta} s \alpha$ for all $s \in S, t \in T$. It then follows that the product map $\theta=\alpha \times \beta: S \bowtie T \rightarrow U \bowtie V$ is a morphism. (Owing to the more complicated multiplication, the calculation required to verify this statement is longer than those in the preceding sections; it makes use of the fact that morphisms of locally inverse semigroups preserve the $\wedge$ operation.)

A routine calculation verifies that the map $i: \bar{X} \rightarrow S \bowtie T$ is matched. If $x \in X$ then $x i \theta=\left({ }^{\left(x x^{\prime}\right) \beta} x_{U}, x_{V}\right)=\left({ }^{x_{V} x_{V}^{\prime}} x_{U}, x_{V}\right)=\left(x_{U}, x_{V}\right)=x \phi$; similarly, $x^{\prime} i \theta=x^{\prime} \phi$. So $i \theta=\phi$. The proof is completed by using Lemma 7.2 in the same way as in the proof of Theorem 7.1.

By Result 10.5, e-free $E$-solid, orthodox and locally inverse semigroups, among others, can therefore all be described in terms of ' $₫$ ' products, by a uniform method. When specialized to the case $\mathbf{V} \subseteq \mathbf{G}$, if $\overline{T \times X}$ is identified with $T \times \bar{X}$ in the manner of $\S 7$, then the theorem is precisely the same as Theorem 7.1. On the other hand, in the case when both $\mathbf{U}$ and $\mathbf{V}$ are locally inverse, our description in Theorem 10.6 is equivalent to that given in [5].

10.3. Comparing the two products. If $T$ is a completely simple semigroup, then for any $u, v \in T$ and $u^{\prime} \in V(u), u(v \wedge u) u^{\prime}$ is an idempotent $\mathcal{H}$-related to, and hence equal to, $u u^{\prime}$. If $T$ acts on a semigroup $S$, then for $(x, u),(y, v) \in S \bowtie T$ we have $(x, u)(y, v)=\left(x^{u} y, u v\right)$, and so $S \bowtie T \leq S * T$. If $S$ is also regular, then $S \bowtie T$ is regular and we obtain the following. (As a consequence of this proposition, some of the inclusions in $\S 3$, such as those of Proposition 3.10, are also valid for the ' $₫$ ' product. For instance, an alternative proof of Result 10.1(v) may be obtained in this way.)

Proposition 10.7. Let $S$ be a regular semigroup and $T$ a completely simple semigroup. Then $S \bowtie T \leq \operatorname{Reg}(S * T)$. Hence for any e-variety $\mathbf{U}$ of regular semigroups and any variety $\mathbf{V}$ of completely simple semigroups, $\mathbf{U} \bowtie \mathbf{V} \subseteq \mathbf{U} * \mathbf{V}$.

In fact, we have seen in the discussion preceding Result 10.5 that $\mathbf{U} \bowtie \mathbf{V}=\mathbf{U} * \mathbf{V}$ if $\mathbf{V}$ is a variety of groups. Note also that from Result 10.5, $L \mathbf{I}=\mathbf{S} \bowtie \mathbf{C S}$. Since, by Corollaries 3.6 and $3.9, \mathbf{S} * \mathbf{C S} \subseteq L(\mathbf{S} * \mathbf{G}) \subseteq L \mathbf{I}$, we have $\mathbf{S} \bowtie \mathbf{C S}=\mathbf{S} * \mathbf{C S}=L \mathbf{I}$ (cf. Result 8.3).

In general, the two products do not coincide, even when the second factor is completely simple. Perhaps the starkest illustration of the difference between the two constructions is the following.

Proposition 10.8. For any e-variety $\mathbf{U}$ and any variety $\mathbf{V}$ of rectangular bands, $\mathbf{U} \bowtie \mathbf{V}=\mathbf{U} \vee \mathbf{V}$.

Proof. Let $S$ be a regular semigroup and $T$ a rectangular band. Note first that $S \times T$ is itself a ' $\bowtie$ '-product, with respect to the action of $T$ on $S$ by identity maps. Thus one inclusion is clear. To prove the other inclusion, it suffices, by Proposition 10.2, to show that the multiplication in $S \bowtie_{\mathrm{wr}} T$ is that of the direct product $S^{T} \times T$. Let $(\alpha, u) \in S \bowtie_{\mathrm{wr}} T$, so that ${ }^{u} \alpha=\alpha$. Then for any $w \in T,{ }^{w} \alpha=\alpha$, since for 
any $t \in T, t\left({ }^{w} \alpha\right)=t\left({ }^{w u} \alpha\right)=(t w u) \alpha=(t u) \alpha=t\left({ }^{u} \alpha\right)=t \alpha$. Making use of the paragraph before Proposition 10.7, the requisite equation follows.

Corollary 10.9. The equations $\mathbf{I} \bowtie \mathbf{R B}=\mathbf{G I}$ and $\mathbf{I} \bowtie \mathbf{R Z}=\mathbf{R G I}$ hold, where RGI is the e-variety of right generalized inverse semigroups (orthodox semigroups whose bands of idempotents are right normal). Hence $\mathbf{I} \bowtie \mathbf{R B} \subset \mathbf{I} * \mathbf{R B}$ and $\mathbf{I} \bowtie \mathbf{R Z} \subset \mathbf{I} * \mathbf{R Z}$.

Proof. The equations follow from [9]. The strict inclusions are immediate from Theorem 4.5, which gives $\mathbf{I} * \mathbf{R Z}=L \mathbf{I}=\mathbf{I} * \mathbf{R B}$.

Remark. In fact, for any nontrivial variety $\mathbf{U}$ of inverse semigroups, $\mathbf{U} \bowtie \mathbf{R Z} \subset$ $\mathbf{U} * \mathbf{R Z}$ (and similarly for $\mathbf{R B}$ ). For we have seen that $\mathbf{U} \bowtie \mathbf{R Z}$ contains only orthodox semigroups. However $\mathbf{U} * \mathbf{R Z}$ contains either $\mathbf{S} * \mathbf{R Z}$ or $\mathbf{H} * \mathbf{R Z}$, for some nontrivial group variety, and thus contains either CSR, by Proposition 4.6, or $L \mathbf{H}$, by Corollary 4.9. But each of these contains a nonorthodox semigroup.

A comparison of Theorems 9.1 (more accurately, the special cases in $\S \S 7,8$ ) and 10.6 leads to the obvious conclusion that the latter's description involves a simpler first factor and a simpler set of generators. In general, however, these advantages are offset by the 'smaller' e-varieties obtained (as evinced by the proposition above, for instance) and the more complicated multiplication in the ' $₫$ ' product. Of course, when the second factor is completely simple, the multiplications coincide. Focusing on the two cases of prime importance, the e-free $E$-solid and locally inverse semigroups, in the former case the descriptions are the same; in the latter case, Theorem 10.6 is apparently simpler - but is dependent on the equation $L \mathbf{I}=\mathbf{S} \bowtie \mathbf{C S}$, which is in turn dependent on Auinger's description of the e-free locally inverse semigroups. On the other hand, Corollary 8.2 is based on the equation $L \mathbf{I}=\mathbf{I} * \mathbf{R Z}$, which was obtained independently via McAlister's theorems $(\S 4)$. Moreover, in $\S 8$ we obtained descriptions of other useful e-free semigroups, for example, the e-free strict regular and combinatorial strict regular semigroups, by replacing $\mathbf{I}$ by various varieties of inverse semigroups, using $\S 4$. Replacing $\mathbf{C S}$ in the equation $L \mathbf{I}=\mathbf{S} \bowtie \mathbf{C S}$ does not yield the same diversity of e-free semigroups.

Perhaps the best conclusion to be drawn is that each product has its uses. The ' $\bowtie$ ' product is defined for all e-varieties of locally inverse semigroups, for instance; on the other hand, the '*'-product also allows arbitrary e-varieties in the second factor (as long as the first is completely simple) — and thus can be applied to products $\mathbf{G} * \mathbf{V}$, as in $\S \S 5.2$ and 8.2 , where the ' $\bowtie$ ' product cannot.

\section{REFERENCES}

[1] J. Almeida, Semidirect products of semigroups from the universal algebraist's point of view, J. Pure Appl. Algebra 60 (1989), 113-128. MR 91a:20068

[2] K. Auinger, The word problem for the bifree combinatorial strict regular semigroup, Math. Proc. Cambridge Phil. Soc. 113 (1993), 519-533. MR 94b:20061

[3] K. Auinger, The bifree locally inverse semigroup on a set, J. Algebra 166 (1994), 630-650. MR 95h:20081

[4] K. Auinger, On the bifree locally inverse semigroup, J. Algebra 178 (1995), 581-613. MR 96i: 20073

[5] K. Auinger and L. Polák, A multiplication of existence varieties of locally inverse semigroups, preprint.

[6] S. Eilenberg, Automata, Languages and Machines, Vol. B, Academic Press, New York, 1976. MR 58:26604b 
[7] T.E. Hall, Some properties of local subsemigroups inherited by larger subsemigroups, Semigroup Forum 25 (1982), 35-49. MR 83m:20082

[8] T.E. Hall, Identities for existence varieties of regular semigroups, Bull. Austral. Math. Soc. 40 (1989), 59-77. MR 90j:20127

[9] T.E. Hall, Regular semigroups: amalgamation and the lattice of existence varieties, Algebra Universalis 29 (1991), 79-108. MR 92g:20098

[10] T.E. Hall, A concept of variety for regular semigroups, in 'Proceedings of the Monash Conference on Semigroup Theory', T.E. Hall, P.R. Jones J. Meakin eds, World Scientific Publ. Co., Singapore, 1991, 101-116. MR 94g:20083

[11] J.M. Howie, An Introduction to Semigroup Theory, Academic Press, London, 1976. MR $\mathbf{5 7 : 6 2 3 5}$

[12] P.R. Jones, Mal'cev products of varieties of completely regular semigroups, J. Austral. Math. Soc. (Ser. A) 42 (1987), 227-246. MR 88a:20069

[13] P.R. Jones, An introduction to existence varieties of regular semigroups, Southeast Asian Bull. Math. 19 (1995), 107-118. MR 96f:20088

[14] P.R. Jones, E-free objects and e-locality of completely regular semigroups, Semigroup Forum 51 (1995), 357-377. CMP 96:01

[15] P.R. Jones and M.B. Szendrei, Local varieties of completely regular monoids, J. Algebra 150 (1992), 1-27. MR 94g:20084

[16] P.R. Jones and P.G. Trotter, Locality of DS and associated varieties, J. Pure Appl. Algebra 104 (1995), 275-301. MR 96i:20074

[17] J. Kadourek, On the word problem for free bands of groups and for free objects in some other varieties of completely regular semigroups, Semigroup Forum 38 (1989), 1-55. MR 89k:20085

[18] J. Kadourek and L. Polák, On the word problem for free completely regular semigroups, Semigroup Forum 34 (1986), 127-138. MR 88a:20066

[19] J. Kadourek and M. Szendrei, A new approach in the theory of orthodox semigroups, Semigroup Forum 40 (1990), 257-296. MR 91k:20075

[20] J. Kadourek and M. Szendrei, On existence varieties of E-solid semigroups, Semigroup Forum, to appear.

[21] S.W. Margolis, Kernels and expansions: a historical and technical perspective, in 'Monoids and Semigroups with Applications', J. Rhodes ed., World Scientific Publ. Co., Singapore, 1991, 1-30. MR 93a:20101

[22] S.W. Margolis and J.-E. Pin, Inverse semigroups and extensions of semilattices by groups, $J$. Algebra 110 (1987), 277 - 297. MR 89j:20066a

[23] D.B. McAlister, Regular Rees matrix semigroups and regular Dubreil-Jacotin semigroups, $J$. Austral. Math. Soc. Ser. A 31 (1981), 325-336. MR 84d:20062

[24] D.B. McAlister, Rees matrix covers for locally inverse semigroups, Trans. Amer. Math. Soc. 277 (1983), 727-738. MR 84m:20073

[25] D.B. McAlister, Rees matrix covers for regular semigroups, J. Algebra 89 (1984), 264-279. MR 86b:20077

[26] W.R. Nico, On the regularity of semidirect products, J. Algebra 80 (1983), 29-36. MR 84i:20064

[27] K.S.S. Nambooripad, The structure of regular semigroups I, Mem. Amer. Math. Soc. 224 (1979). MR 81i:20086

[28] H. Neumann, Varieties of Groups, Springer-Verlag, Berlin, 1967. MR 35:6734

[29] F. Pastijn, The lattice of completely regular semigroup varieties, J. Austral. Math. Soc. (Ser A) 49 (1990), 24-42. MR 91d:20068

[30] F. Pastijn and P.G. Trotter, Lattices of completely regular semigroup varieties, Pacific J. Math. 119 (1985), 191-224. MR 87b:20079

[31] M. Petrich, Inverse Semigroups (Wiley, New York, 1984). 59-74. MR 85k:20001

[32] L. Polák, A multiplication on the lattice of varieties of *-regular semigroups, in "Semigroups", C. Bonzini, A. Cherubini and C. Tibiletti, eds, World Sci. Publ. Co., Singapore, 1993, 231245

[33] M. Szendrei, Orthogroup bivarieties are bilocal, in 'Semigroups With Applications', J.M. Howie, W.D. Munn and H.J. Weinert eds, World Scientific Publ. Co., Singapore, 1992, 114131. MR 93j:20131 
[34] M. Szendrei, On E-unitary covers of orthodox semigroups, Int. J. Alg. Comp. 3 (1993), 317-334. MR 95b:20093

[35] M. Szendrei, The bifree regular E-solid semigroups, Semigroup Forum 52 (1996), 61-82. MR 96m:20099

[36] L.A. Skornyakov, Regularity of the wreath product of monoids, Semigroup Forum 18 (1979), 83-86. MR 80e: 20077

[37] B. Tilson, Categories as algebra: an essential ingredient in the theory of monoids, J. Pure Appl. Algebra 48 (1987), 83-198. MR 90e:20061

[38] P.G. Trotter, Congruence extensions in regular semigroups, J. Algebra 137 (1991), 166-179. MR 92e:20045

[39] P.G. Trotter, Covers for regular semigroups and an application to complexity, J. Pure Appl. Algebra 105 (1995), 319-328. MR 96m:20100

[40] P.G. Trotter, Relatively free bands of groups, Acta Sci. Math. (Szeged) 53 (1989), 19 - 31. MR 91d:20067

[41] P.G. Trotter, E-varieties of regular semigroups, in Semigroups, Automata and Languages, J. Almeida, G.M.S. Gomes and P.V. Silva eds, World Scientific Publ. Co., Singapore, 1994, $247-262$.

[42] Y.T. Yeh, The existence of e-free objects in e-varieties of regular semigroups, Int. J. Alg. Comp. 2 (1992), 471-484. MR 94d:20085

[43] S. Zhang, An infinite order operator on the lattice of varieties of completely regular semigroups, Algebra Universalis 35 (1996), 485-505. MR 97e:20082

[44] S. Zhang, Completely regular semigroup varieties generated by Mal'cev products with groups, Semigroup Forum 48 (1994), 180-192. MR 95a:20063

Department of Mathematics, Statistics and Computer Science, Marquette UniverSity, P.O. Box 1881, Milwaukee, Wisconsin 53201-1881

E-mail address: jones@mscs.mu.edu

Department of Mathematics, University of Tasmania, Hobart, Australia, 7001

E-mail address: trotter@hilbert.maths.utas.edu.au 